\title{
Generalized multinomial CRR option pricing model and its Black-Scholes type limit
}

\author{
Dissertation \\ zur Erlangung des Doktorgrades \\ der Mathematisch-Naturwissenschaftlichen Fakultäten \\ der Georg-August-Universität zu Göttingen
}

vorgelegt von

Natalia Kan

aus

Taschkent, Uzbekistan

Göttingen 2005 
D7

Referent: Prof. Dr. Manfred Denker

Korreferentin: Prof. Dr. Jeannette Woerner

Tag der mündlichen Prüfung: 09.11.2005 
To family, friends, mentors 


\section{Contents}

Introduction $\quad 6$

1 Generalized Cox-Ross-Rubinstein Model 15

1.1 Generalized Cox-Ross-Rubinstein Model of a Stock Price . . . . . 15

1.2 Finite Spot Markets. Definitions. . . . . . . . . . . . . . . . 18

1.3 Generalized CRR Arbitrage Option Pricing . . . . . . . . . . . . . 20

1.4 Random sets. Auxiliary results . . . . . . . . . . . . . . 24

1.5 Generalized CRR Option Pricing Formula . . . . . . . . . . 26

2 Conditional Generalized Cox-Ross-Rubinstein Model 31

2.1 Conditional CRR Option Price . . . . . . . . . . . . . . 31

2.2 Multinomial model. Definitions . . . . . . . . . . . . . . 34

2.3 Multinomial option pricing . . . . . . . . . . . . . . . 37

3 Convergence of Generalized Multinomial Option Price Model 47

3.1 Averaging over all possible multinomiality parameters sets . . . . 48

3.2 Asymptotic procedure . . . . . . . . . . . . . . . . . 49

3.3 Multinomial CRR volatility modelling . . . . . . . . . . . . 51

3.4 Limit theorems for multinomial distribution. Auxiliary results . . 53

3.5 Generalized Multinomial Black-Scholes option pricing formula . . 57

4 Numerical Results $\quad 64$

4.1 Stock Price Modelling. Hull-White Algorithm . . . . . . . . . . . 65

4.2 Stock Price Modelling. Estimation of multinomiality parameters . 66

4.3 The Stock Price Modelling. Simulations . . . . . . . . . . . . . . 67

4.4 Numerical option price calculation. Multinomial CRR Model . . 71 
4.5 Generalized multinomial Black-Scholes formula. Simulations . . . 76

Bibliography

79

Curriculum Vitae 


\section{Introduction}

Financial markets are basic structures of financial theory, play the central role among the other structures and are of the primary concern for mathematical theory of finance.

In this thesis we consider the markets of financial instruments or securities where one usually distinguishes underlying (primary) instruments and derivative (secondary) instruments. Derivative securities are constructed on the basis of the underlying instruments.

Under underlying financial instruments one usually considers the bank accounts, bonds, stocks. As derivative financial instruments one considers options, futures, contracts, warrants, swaps, combinations, etc. In more detail about financial markets and their instruments one is referred to [60], [48], [28], [52].

The main purpose of derivative securities construction is to manipulate with them to satisfy the natural wish of investors to raise capital and reduce risks caused by the uncertain character of the market behavior in the future.

It is worthwhile to notice that options are very important derivative securities in financial markets and not only. Virtually, almost all corporate securities can be interpreted as portfolios of put and call options written on the stocks of the firm and therefore the option pricing theory finds a use for practically all areas

in finance. So, the problem of option pricing is one of the central problems in financial mathematics.

As call option we understand the right, without the obligation, to buy in the future a share of stock for a guaranteed price.

As put option we understand the right, without the obligation, to sell in the future a share of stock for a guaranteed price correspondingly.

Numerous researchers have continued to contribute to the theory of option 
pricing already after 1900 when French mathematician Louis Bachelier derived an option pricing formula with the assumption that underlying stock price follows a Brownian motion. So that the theory of option pricing has been and remains important nowadays.

Options themselves have been traded for a long time and at the same time they have been remained as relatively vague financial instruments. It was so until 1973 when the option exchange has been introduced. And at the same 1973 the option pricing theory underwent a revolutionary changing.

In that year Fischer Black and Myron Scholes [7] offered first satisfactory model of option pricing. Then Robert Merton [44] extended this model in some important ways. After these results many subsequent studies have been done. It was shown that option pricing theory is relevant to almost every area of finance. One should notice that the mathematical tools Black, Scholes [7] and Merton [44] employed are quite advanced and this fact doesn't make the underlying economics more clear.

In 1978 Sharpe [63] has partially developed the simplified approach to option pricing and suggested the advantages of using the discrete-time approach to option pricing.

Within this setting the fundamental economic principles of option valuation by arbitrage methods become particularly clear.

In 1979 Cox, Ross and Rubinstein [20] presented a discrete-time option pricing formula. Among their numerous and wholesome developed results are the model for a call option on a stock which doesn't pay any dividends and the explanation that their approach includes the Black-Scholes [7] model as a special limit case. Many other problems of option pricing don't come out with the closed formula. To value these more complex options numerical procedures are suggested. And again complicated techniques are used which are not directly related to the economic structure of the problem.

Quoting Cox, Ross and Rubinstein [20], "Our formulation, by its very construction, leads to an alternative numerical procedure which is both simpler, and for many purposes, computationally more efficient.", it is necessary to notice that this formulation suggests itself to be extended in some ways. Madan, Milne and Shefrin [36] considered the extension of the binomial Cox-Ross-Rubinstein [20] model to the multinomial case. Where "economic uncertainty is modeled as 
evolving on an $(n+1)$-ary tree with branching occurring during a short interval of time in which there is no trading." Initially, Madan et al. [36] consider the multinomial option pricing model for an arbitrary $(n+1)$-ary branching tree and then obtain the limit option value as Black-Scholes formula.

R. Merton in 1976 [45] considered the problem of option pricing when underlying stock returns are discontinuous.

In 1991 [57] some exotic options pricing is considered, one example of exotic options is a compound option. The compound option is like a standard option except that its underlying asset is itself a standard option.

Another kind of exotic options is "Russian Option", its pricing valuation is studied in [64], [65], [21], [32].

Good review of basic aspects, statements of problems and results of financial mathematics in connection with the option pricing theory in modern presentation is given in [66], [67]

Mainly, the "martingale" methods of option pricing are used. The idea of martingale methods is that the underlying security process is assumed to be a martingale with respect to some measure which reflects the conception of fair game in the market. The work of Rachev and Rüschendorf [53] should be mentioned where all possible stock price models have been characterized, these models can be approximated by the binomial models and the corresponding approximations for the pricing formulas have been obtained. Also, two additional randomizations in the binomial price models were introduced in order to find more general and more realistic limiting models. This randomization consists of the considering the random number of stock price changes and the assumption, that the values of stock price jumps are random values but they do not change with time.

In 1996 Rejman and Weron [54] have employed the idea of Rachev and Rüschendorf to generalize Cox-Ross-Rubinstein (1979) binomial model. They have found the limiting model assuming that the stock returns follow hyperbolic distribution.

Another generalization of Cox-Ross-Rubinstein (1979) has been done by Motoczyński and Stettner in 1998. They considered the option pricing in multidimensional case with a number of risky assets, which are in discrete time subject to binomial disturbances. Also, multidimensional case was considered by Tessitore and Zabczyk in 1991 [71]. They have derived formulaes for option prices when 
the financial market is incomplete.

Along with these generalizations of Cox-Ross-Rubinstein model there is a big discussion about how financial asset returns should be modeled.

The problem of stock returns data modeling is closely related to the problem of data approximation. There are several approaches to this problem and one of them is the distribution based approach.

The distribution based concept of stock price data approximation has been considered by many researches and it has been continued until nowadays.

It is well known that in continuous-time modeling the normality of assets played a central role in financial theory. Samuelson [58] introduced in 1964 the Geometric Brownian motion, then the normality of asset returns is used in the seminal papers by Black-Scholes [7] and Merton [44].

For some time past and as documented in considerable number of papers [1], [2], [24], [34], [35], [40], [46], [50], [70], [29], [18] written by academics and practitioners, both normality and continuity assumptions are contradicted by the most of real data.

It is known that the volatility parameter serves as a variance measure of the financial market and plays an important role in option pricing valuation. The Black-Scholes formula was obtained under the assumptions that the volatility is constant which in many cases does not correspond to reality and by empirical studies it was shown that there is a phenomena of so called volatility smile when the implied volatility in dependence on the strike price (specified guaranteed price of a share of stock on the expiration date) of the option has a smile shape.

Since the assumptions about the normality of underlying stock price and constant volatility parameter are not satisfactory, many researchers try to mend the Black-Scholes theory adding various frictions and distortions to deduce the arising volatility smile. Or, they assume that stock returns follow some kinds of Lévy processes.

In 1963 Mandelbrot [41] suggested to use Lévy stable distributions to model the returns in the financial markets. In 1965 Fama [24] noted that asset returns distributions are more leptokurtic than normal; this feature is more accentuated when the holding period becomes shorter and becomes clear on high frequency data. 
There is some historical review about what kind of research has been done concerning stock returns modeling and option pricing with respect to distribution based conception of data approximation. From the beginning of 90th, several families of Lévy processes with probability densities having semi-heavy, that is, exponentially decaying tails have been used to model stock returns and price options: Variance Gamma Processes (VGP), used by D. Madan with co-authors [37], [38], Normal Inverse Gaussian Processes (NIG), used by O.E. BarndorffNielsen's group [3], Hyperbolic Processes and Generalized Hyperbolic Processes (HP and GNP) used by Eberlein's group [22], Truncated Lévy Processes (TLP), constructed by Koponen [31], used by J.-P. Bouchaud and his group [9], [10], [11] and extended by Boyarchenko and Levendovski [12], [13], [14] and Normal Tempered Stable Lévy Processes (NTS Lévy Processes) [4], [5].

As it is shown, this is very important properly to approximate the data of underlying risky security to obtain pertinent corresponding option prices. One can expect that if the good stochastic model of approximation for stock price process is found, then it is more likely to obtain more reasonable option prices.

It is not evident that real data should follow some certain known distribution specified by the expert. So, we use the different approach to data approximation, namely, we construct a model which allows to exhibit the most features of data and minimize the distance between real and modeled data. Evidently, the stock price follows the up and down process which has been well described by classical random walk process. But, the option prices calculated using the classical results of Cox et al. ( [20]) (CRR option pricing formula) and famous Black-Scholes formula ( [7]) started to fail quite often. So, in this context, it seems to be plausible to construct the extension of Cox-Ross-Rubinstein binomial model and consider the possible limits of new extended model.

Mostly in practice the discrete model of option pricing is preferred. It is explained by the fact that Black-Scholes formula is a particular limit case of discrete binomial Cox-Ross-Rubinstein model( [20]). In other words, the binomial model provides discrete approximations to the continuous price process underlying in the Black-Scholes model.

The classical discrete Cox-Ross-Rubinstein model of financial market is de- 
fined by a saving account or a bond process

$$
B_{t}=(1+r)^{t}=\hat{r}^{t}, \quad \forall \quad t \leq T^{*},
$$

where $r \geq 0$ is a constant rate of return and $B_{0}=1$.

The stock price process in classical discrete Cox-Ross-Rubinstein model is given by

$$
S_{t}=\xi_{t-1} S_{t-1}, \quad \forall \quad t \leq T^{*},
$$

where $\xi_{t}, t \leq T^{*}$ are independent identically distributed random variables taking two possible values $u$ and $d$ with probabilities $p$ and $1-p$ respectively. And $d<1+r<d$ are given real numbers, $S_{0}$ is strictly positive constant.

Since Black-Scholes model is often not satisfactory in practice anymore and classical Cox-Ross-Rubinstein is the approximation for Black-Scholes, it is not enough to be pleased only with classical Cox-Ross-Rubinstein model too.

So, in this thesis, we consider the following problems to solve

- (1) to construct an alternative (to binomial one) model of the underlying security of call option which fits (approximates) data better then the binomial model with respect to the values of stock price jumps having changed with time

- (2) to obtain the generalization of Cox-Ross-Rubinstein option price with respect to the alternative model of the stock price process

- (3) to obtain the possible limit of generalized Cox-Ross-Rubinstein option price

The dissertation consists of four chapters, one of which contains numerical results and corresponding graphs.

In the first chapter of dissertation we construct the generalized discrete-time model of the underlying stock price process which can be better approximation to the stock price process than classical random walk.

Definition 1.1.1 The model of stock price process is called generalized 
Cox-Ross-Rubinstein stock price model if $S$ is defined as follows

$$
S_{t}=S_{t-1} \xi_{t-1}, \quad \forall \quad t \leq T^{*}
$$

where $S_{1}=S_{0} \xi_{0}, S_{0}$ is given positive constant and

$$
\xi_{t}=X_{t} \nu_{t}, \quad \forall \quad t \leq T^{*}
$$

where $\left\{\nu_{t}\right\}_{t \leq T^{*}}$ are Bernoulli random variables taking values $u$ and $d$ with corresponding probabilities $p$ and $1-p$. Also assume that random variables $\left\{X_{t}\right\}_{t \leq T^{*}}$ and $\left\{\nu_{t}\right\}_{t \leq T^{*}}$ are mutually independent.

We also derive the corresponding generalized model for option price. The technique used to derive the option price is the backward induction technique which first was suggested by Sharpe in 1978 [63].

Proposition 1.5.1. The arbitrage price of a European call option at time $t=T-m$ is given by the following formula

$$
C_{T-m}=S_{T-m} \sum_{j=0}^{m}\left(\sum_{J \in I_{j, m}\left(S_{T-m}\right)} \bar{P}\left(J^{(j)}, T\right)-\frac{K}{\hat{r}^{m}} \sum_{J \in I_{j, m}\left(S_{T-m}\right)} P\left(J^{(j)}, T\right)\right)
$$

for $m=0, \ldots, T$, where

$$
\begin{aligned}
\bar{P}\left(J^{(j)}, T\right) & :=\prod_{k \in J} \bar{p}_{T-k} \prod_{k \notin J} \bar{q}_{T-k}, \\
P\left(J^{(j)}, T\right) & :=\prod_{k \in J} p_{T-k} \prod_{k \notin J} q_{T-k},
\end{aligned}
$$

and

$$
p_{T-k}:=\frac{\hat{r}-\xi_{T-k}^{d}}{\xi_{T-k}^{u}-\xi_{T-k}^{d}}, \quad \bar{p}_{T-k}:=\frac{\xi_{T-k}^{u}}{\hat{r}} p_{T-k}, \quad q_{T-k}=1-p_{T-k}, \quad \bar{q}_{T-k}=1-\bar{p} .
$$

At time $t=T-m-1$ the unique replicating strategy $\phi$ is correspondingly determined.

In the second chapter we impose specific assumptions (constrains) on the new model of stock price process and as a result the multinomial Cox-Ross-Rubinstein 
model for option price is obtained. This model is called multinomial because the multinomial coefficients emerge from these specific assumptions.

Proposition 2.3.1 If the condition

$$
\mathbf{P}\left\{X_{i} \in \mathbb{C}_{k}\right\}=1, \quad \forall i=T-m, \ldots, T
$$

is satisfied, then

$$
\begin{gathered}
\tilde{C}_{T-m}(c)=k^{m} \sum_{j=a}^{m} \sum_{I\left(m, j, N_{c_{1}}, \ldots, N_{c_{k}}\right)}\left(S_{T-m} M_{2 k}(m, \bar{p})-\frac{K}{\hat{r}^{m}} M_{2 k}(m, p)\right), \\
\forall \quad c=\left(c_{1}, \ldots, c_{k}\right) \in \mathbb{R}_{+}^{k},
\end{gathered}
$$

where

$$
\begin{gathered}
p_{1}\left(c_{1}\right)=\frac{1}{k} \frac{\hat{r}-c_{1} d}{c_{1}(u-d)}, \ldots, p_{k}\left(c_{k}\right)=\frac{1}{k} \frac{\hat{r}-c_{k} d}{c_{k}(u-d)}, \\
p_{k+1}\left(c_{1}\right)=\frac{1}{k} \frac{c_{1} u-\hat{r}}{c_{1}(u-d)}, \ldots p_{2 k}\left(c_{k}\right)=\frac{1}{k} \frac{c_{k} u-\hat{r}}{c_{k}(u-d)} \\
\bar{p}_{1}=\frac{c_{1} u}{\hat{r}} p_{1}, \ldots, \bar{p}_{k}=\frac{c_{k} u}{\hat{r}} p_{k}, \quad \bar{p}_{k+1}=\frac{c_{1} d}{\hat{r}} p_{k+1}, \ldots, \bar{p}_{2 k}=\frac{c_{k} d}{\hat{r}} p_{2 k}
\end{gathered}
$$

and

$$
\begin{aligned}
& p_{1}+p_{k+1}=\ldots=p_{k}+p_{2 k}=\frac{1}{k}, \\
& \bar{p}_{1}+\bar{p}_{k+1}=\ldots=\bar{p}_{k}+\bar{p}_{2 k}=\frac{1}{k} .
\end{aligned}
$$

The quantities $a=a\left(S_{T-m}\right), M_{2 k}(m, \bar{p}), M_{2 k}(m, p)$ and sets $I\left(m, j, N_{c_{1}}, \ldots, N_{c_{k}}\right.$, $\mathbb{C}_{k}$ are defined in the section 2.2

Also, we deal with so called "multinomiality parameters" in the model which have to be estimated and they are of the main interest of discussion in chapter four where some estimation procedure is proposed.

In the third chapter we construct asymptotic procedure and consider a limit of multinomial Cox-Ross-Rubinstein model. As a result we obtain generalized Black-Scholes option pricing formula dependent on the multinomiality parameters.

Theorem 3.5.1. The following convergence is valid for any dyadic $t \in[0, T]$

$$
\lim _{n \rightarrow \infty} \hat{C}_{T-m}=\hat{C}_{t}=S_{t} \Phi\left(f_{1}\left(S_{t}, T-t\right)\right)-K e^{-r(T-t)} \Phi\left(f_{2}\left(S_{t}, T-t\right)\right)
$$


where

$$
\begin{gathered}
f_{1}(s, t)=\frac{\ln \frac{s}{K}+(T-t) \frac{\ln c_{1} \ldots c_{k}+k}{k}\left(\frac{r}{k} \frac{\ln c_{1} \ldots c_{k}+k}{\prod_{i=1}^{k}\left(\ln c_{i}+1\right)}+\frac{\sigma^{2}}{2} \frac{\ln c_{1} \ldots c_{k}+k}{k}\right)}{\sigma \frac{\ln c_{1} \ldots c_{k}+k}{k} \sqrt{T-t}}, \\
f_{2}(s, t)=f_{1}(s, t)-\sigma \frac{\ln c_{1} \ldots c_{k}+k}{k} \sqrt{T-t}
\end{gathered}
$$

and $\Phi$ stands for the standard Gaussian cumulative distribution function $\Phi(x)=\frac{1}{\sqrt{2 \pi}} \int_{-\infty}^{x} e^{\frac{u^{2}}{2}} d u, \quad \forall x \in \mathbb{R}$.

In the fourth chapter we describe multinomility parameters estimation procedure and introduce numerical results with corresponding graphs showing the advantages of new model.

We will index definitions, theorems and lemmas in the following way: the first number will refer to the chapter and the second number will refer to the section of the chapter and the third number will refer to their number in the section. The same holds for numbering of equations and formulas.

\section{Acknowledgements}

I would like to express my deep gratitude to my supervisor, Prof. Dr. Manfred Denker, for proposing the topic of my dissertation and his excellent supervision. I am also grateful to my coadvisor Prof. Dr. Jeannette Woerner for excellent explanation of issues of financial mathematics and being a Korreferentin. Furthermore, I thank Prof. Dr. Walter Zucchini for his helpful and motivating discussions.

I would like to thank all people from the Institute for Mathematical Stochastics for creating a warm atmosphere which above all supports scientific research. Thanks go to Prof. Dr. Axel Munk, Prof. Dr. Susanne Koch, Dr. Gudrun Freitag, Dr. Manuel Stadlbauer, Janis Valeinis, Achim Wübker, Mohammed Abu Jarad and all my other colleagues for their friendship and for the beautiful time spent outside the IMS.

Especially, I would like to thank Michael and my family for supporting me with great patience and loving care throughout my life.

Financial support from the Center for Statistics is gratefully acknowledged. 


\section{Chapter 1}

\section{Generalized Cox-Ross-Rubinstein Model}

In 1978 Sharpe [63] and in 1979 Rendleman and Bartter [55] independently developed the two-state option pricing model. The main idea is to construct a portfolio at time 0 which replicates the option's terminal payoff at time $T$. Classical Cox-Ross-Rubinsten [20] model is based on two-state stock price process whose dynamics is modeled by multiplicative binomial lattice.

We propose a new generalized form of Cox-Ross-Rubinstein model for the valuation of European call option price at any instant $t=0, \ldots, T^{*}$, when the risky asset (a stock price) binomial model is naturally randomized when at each time moment the jumps of upward and downward movements have different sizes.

\subsection{Generalized Cox-Ross-Rubinstein Model of a Stock Price}

We assume that the securities market operates under conditions of "uncertainty" which can be described in terms of probability space $(\Omega, \mathcal{F}, \mathbb{P})$. A discrete-time model of a financial $(B, S)$-market during the time interval $\left[0, T^{*}\right]=\left\{0, \ldots T^{*}\right\}$ is considered, where $T^{*}$ is some positive natural number. It is denoted as $(B, S)$ market because there are two primary traded securities at this market: a risky asset, $S$, referred to as a stock and a risk-free investment, $B$, called a savings account (or bond). 
The first security is a stock whose price process is modelled as a strictly positive discrete-time process $S=\left(S_{t}\right)_{t \leq T}$, it is assumed that $S_{t}$ is $\mathcal{F}_{t}$-adapted, i.e. random variables $S_{t}$ are $\mathcal{F}_{t}$-measurable for $t \in[0, T]$, the structure of sigma algebras $\mathcal{F}_{t}$ will be described later.

The price process of a bond (risk-free investment) is defined as

$$
B_{t}=(1+r)^{t}, \quad \forall \quad t \leq T^{*},
$$

where $\hat{r}=1+r, r$ is any positive real number.

Definition 1.1.1 The model of stock price process is called generalized Cox-Ross-Rubinstein stock price model if $S$ is defined as follows

$$
S_{t}=S_{t-1} \xi_{t-1}, \quad \forall \quad t \leq T^{*}
$$

where $S_{1}=S_{0} \xi_{0}, S_{0}$ is given positive constant and

$$
\xi_{t}=X_{t} \nu_{t}, \quad \forall \quad t \leq T^{*},
$$

where $\left\{\nu_{t}\right\}_{t \leq T^{*}}$ are Bernoulli random variables taking values $u$ and $d$ with corresponding probabilities $p$ and $1-p$. Also assume that random variables $\left\{X_{t}\right\}_{t \leq T^{*}}$ and $\left\{\nu_{t}\right\}_{t \leq T^{*}}$ are mutually independent.

Remark 1.1.2 The following relation has to be satisfied

$$
0<d<1+r<u \text {. }
$$

Within this model we don't impose any additional assumptions on the sequence of random variables $\left\{X_{t}\right\}_{t \leq T^{*}}$ except the independence on $\left\{\nu_{t}\right\}_{t \leq T^{*}}$ and that $\mathbb{P}\left\{X_{t}>0\right\}=1$ for any $t=1, \ldots, T^{*}$.

We can write down (1.1.2) as

$$
S_{t}=S_{t-1} X_{t-1}\left(u \eta_{t}+d\left(1-\eta_{t}\right)\right), \quad t \leq T^{*},
$$

where random variables $\eta_{t}, \quad t \leq T^{*}$ are independent identically distributed Bernoulli random variables which take values 0 and 1 with probabilities $1-p$ and $p$ respectively. From (1.1.2) we have that

$$
S_{t}=S_{0} \prod_{i=1}^{t-1} \xi_{i}, \quad t \leq T^{*} .
$$


By assumptions have been done above random variables $\xi_{t}, t \leq T^{*}$ are mutually independent random variables on common probability space $(\Omega, \mathcal{F}, \mathbb{P})$ with probability law

$$
\begin{aligned}
& \mathbb{P}\left\{\xi_{t}=x u\right\}=\mathbb{P}\left\{X_{t} \nu_{t}=x u \mid X_{t}=x\right\}=p=1-\mathbb{P}\left\{X_{t} \nu_{t}=x d \mid X_{t}=x\right\} \\
& =1-\mathbb{P}\left\{\xi_{t}=x d\right\}, \quad \forall \quad t \leq T^{*}
\end{aligned}
$$

Notice that the stock price model (1.1.4) is equivalent to

$$
S_{t}=S_{0} \prod_{i=1}^{t-1} X_{i} \exp \left\{\sum_{i=1}^{t-1} \zeta_{i}\right\}, \quad t \leq T^{*}
$$

where $\zeta_{t}, t \leq T^{*}$ are independent identically distributed random variables such that

$$
\mathbb{P}\left\{\zeta_{t}=\ln u\right\}=p=1-\mathbb{P}\left\{\zeta_{t}=\ln d\right\}, \quad \forall \quad t \leq T^{*}
$$

Remark 1.1.3 The model (1.1.3) explains that at any time moment the stock price is changed not only by multiplication by the factors of two possible values $u$ and $d$ but due to the embedding of the process $\left\{X_{t}\right\}_{t \leq T^{*}}$, the changes of stock price are modeled by variety of possible values $u X_{t}$ and $d X_{t}, t \leq T^{*}$ according to the assumptions on the random sequence $\left\{X_{t}\right\}_{t \leq T^{*}}$.

The stock price process $S$ given by (1.1.2) generates the family of natural $\sigma$-fields

$$
\mathcal{F}_{t}^{S}=\sigma\left(S_{0}, S_{1}, \ldots, S_{t}\right), \quad \forall \quad t \leq T,
$$

where $\sigma\left(S_{0}, \ldots, S_{t}\right)$ denotes the least $\sigma$-field with respect to which random variables $S_{0}, \ldots, S_{t}$ are measurable. We write $\left(\mathcal{F}_{t}^{S}\right)_{t \leq T}$ to denote the "information flow" accessible to all participants of the market.

Throughout the chapter we use the following notations for

$$
\xi_{t}^{u}:=X_{t} u \quad \text { and } \quad \xi_{t}^{d}:=X_{t} d
$$

We will abbreviate Cox-Ross-Rubinstein as CRR hereinafter. 


\subsection{Finite Spot Markets. Definitions.}

We consider a European call option written on one share of stock $S$, which doesn't pay any dividends during the option's lifetime. This option is equivalent to the claim $Y$ with payoff at time $T, T \leq T^{*}$, where $T$ denotes an exercise time of a call, contingent on the stock price $S_{T}$.

$$
Y=\left(S_{T}-K\right)^{+} \stackrel{\text { def }}{=} \max \left\{S_{T}-K, 0\right\}
$$

where $K$ is the exercise price of the option. The call option value at the expiry time $T$ simply equals $C_{T}=\left(S_{T}-K\right)^{+}$. We want to evaluate the option price $C_{t}$ at any time moment $t=0, \ldots, T$.

First, we describe the investor's portfolio and how the corresponding replication strategy is constructed.

Definition 1.2.1. [60] (pp.384) A predictable stochastic sequence $\phi=(\alpha, \beta)$, where $\alpha=\left(\alpha_{t}(w)\right)_{t \geq 0}$ and $\beta=\left(\beta_{t}(w)\right)_{t \geq 0}$ with $\mathcal{F}_{t-1}$-measurable $\alpha_{t}(w)$ and $\beta_{t}(w)$ for all $t \geq 0\left(\mathcal{F}_{-1}=\mathcal{F}_{0}\right)$ is called an investment portfolio on the $(B, S)$-market.

For any $t \leq T$, we interpret $\alpha_{t}$ as the number of stock shares held during the period $[t, t+1)$ and $\beta_{t}$ stands for the dollar investment in the savings account during this period. Sometimes the investment portfolio is called an investment or trading strategy instead.

Here the investment strategy $\phi_{t}$ has to be determined on the basis of information available before time $t$, which means that investor selects his portfolio $\phi_{t}$ after observing the prices $S_{t-1}$.

Definition 1.2.2. [60] (pp.385) The value of an investment portfolio $V$ at time $t$ is the stochastic sequence

$$
V(\phi)=\left(V_{t}(\phi)\right)_{t \geq 0}
$$

where

$$
V_{t}(\phi)=\alpha_{t} S_{t}+\beta_{t}
$$

and $V_{0}(\phi)=\alpha_{1} S_{0}+\beta_{1}$. The process $V_{t}(\phi)$ is called the wealth of the trading strategy $\phi$. 
Denote as $\Phi$ a linear space of all stock-bonds portfolios $\phi$, then consider a security market model $\mathcal{M}=(B, S, \Phi)$.

Definition 1.2.3. [25] (pp.210) A trading strategy $\phi$ is called self-financing if

$$
\alpha_{t-1} S_{t}+\beta_{t-1} \hat{r}=\alpha_{t} S_{t}+\beta_{t}, \quad \forall \quad t \leq T^{*} .
$$

Intuitively, (1.2.3) means that the portfolio is recombined in such a way that its value remains the same. When new prices are quoted at time $t$, the investor adjusts his portfolio from $\phi_{t-1}$ to $\phi_{t}$ without any withdrawals or inputs of funds concerning the wealth of portfolio.

Definition 1.2.4. [48] (pp.15) A security pricing model $\mathcal{M}$ is called arbitrage-free if there is no portfolio $\phi \in \Phi$ for which

$$
V_{0}(\phi)=0, \quad V_{T}(\phi) \geq 0 \quad \text { and } \quad \mathbb{P}\left\{V_{T}(\phi)>0\right\}>0 .
$$

A portfolio $\phi$ for which conditions (1.2.4) are satisfied is called an arbitrage opportunity.

Definition 1.2.5. Portfolio $\phi$ for which the conditions

$$
V_{0}(\phi)<0, \quad \mathbf{P}\left\{V_{T}(\phi) \geq 0\right\}=1
$$

are satisfied is called a strong arbitrage opportunity.

In other words, the arbitrage opportunity is a self-financing strategy with zero initial portfolio value and which produces a non-negative terminal portfolio value with probability one and has a positive terminal value with positive probability.

We introduce a conception of an arbitrage price meaning that the price is derived when no arbitrage opportunities are possible. We shall consider the pricing of contingent claim $Y$ of a European call option in a security market $\mathcal{M}$. By a European contingent claim $Y$ which is paid off at time $T$ we mean an arbitrary $\mathcal{F}_{T}$-measurable random variable. 


\subsection{Generalized CRR Arbitrage Option Pricing}

Definition 1.2.6 A replicating strategy for the contingent claim $Y$ which is paid off at time $T$, is a self-financing trading strategy $\phi$ such that $V_{T}(\phi)=Y$

The replicating strategy can be not unique. There is usually a class of trading strategies which replicate $Y$.

Definition 1.2.7 We say that a contingent claim $Y$ is attainable in $\mathcal{M}$ if it admits at least one replicating strategy.

Definition 1.2.8 A market $\mathcal{M}$ is called complete if any contingent claim $Y$ is attainable in $\mathcal{M}$, or, equivalently, if for every $\mathcal{F}_{T}$-measurable random variable $Y$, where $T$ is expiration time for a claim $Y$, there exists at least one trading strategy $\phi \in \Phi$ such that $V_{T}(\phi)=Y$.

The completeness of the market model is very desirable property. Only under market completeness, any European claim can be priced by arbitrage and its price process can be replicated by means of a replicating self-financing strategy.

Definition 1.2.6. [48] (pp.15) Suppose that the security market $\mathcal{M}$ is arbitrage-free. Then the rational price of $Y$ is called the arbitrage price of $Y$.

\subsection{Generalized CRR Arbitrage Option Pricing}

To find the arbitrage price of a call option based on the model (1.1.2) defined in Definition 1.1.1 we will use a method of backward induction [48] which shows that if the investor properly adjusts the portfolio $\phi_{t}=\left(\alpha_{t}, \beta_{t}\right), t \leq T$ at the beginning of each time period, then at every time moment it is possible to mimic the payoff of an option at time $T$. This means that the contingent claim $Y$ defined in (1.2.1) admits a unique, dynamic, replicating, self-financing strategy. The constant $K>0$ and the fixed maturity date $T, 1 \leq T \leq T^{*}$ are given.

We start the analysis by considering the last period before the expiration date, i.e. the time interval $[T-1, T]$. Assume that a portfolio which replicates the terminal call option payoff is determined at time $T-1$ and remains 


\subsection{Generalized CRR Arbitrage Option Pricing}

unchangeable until the expiry date $T$. It means that we need to find the composition of a portfolio $\phi_{T-1}=\left(\alpha_{T-1}, \beta_{T-1}\right)$ in such a way that it's terminal wealth $V_{T}(\phi)$, which is defined as

$$
V_{T}(\phi)=\alpha_{T-1} S_{T}+\beta_{T-1} \hat{r}
$$

replicates the option payoff $C_{T}$, i.e. $V_{T}(\phi)=C_{T}$. So,

$$
\alpha_{T-1} S_{T}+\beta_{T-1} \hat{r}=\left(S_{T}-K\right)^{+} .
$$

According to (1.1.2) and (1.1.6) we have $S_{t}=S_{t-1} \xi_{t-1}^{u}$ with probability $p$ or $S_{t}=S_{t-1} \xi_{t-1}^{d}$ with probability $1-p$; therefore, we can write the following system of equations

$$
\left\{\begin{array}{l}
\alpha_{T-1} \xi_{T-1}^{u} S_{T-1}+\beta_{T-1} \hat{r}=\left(\xi_{T-1}^{u} S_{T-1}-K\right)^{+} \\
\alpha_{T-1} \xi_{T-1}^{d} S_{T-1}+\beta_{T-1} \hat{r}=\left(\xi_{T-1}^{d} S_{T-1}-K\right)^{+}
\end{array} .\right.
$$

This system of equations is easily solved with respect to $\alpha_{T-1}$ and $\beta_{T-1}$

$$
\begin{gathered}
\alpha_{T-1}=\frac{\left(\xi_{T-1}^{u} S_{T-1}-K\right)^{+}-\left(\xi_{T-1}^{d} S_{T-1}-K\right)^{+}}{S_{T-1}\left(\xi_{T-1}^{u}-\xi_{T-1}^{d}\right)} \\
\beta_{T-1}=\frac{\xi_{T-1}^{u}\left(\xi_{T-1}^{d} S_{T-1}-K\right)^{+}-\xi_{T-1}^{d}\left(\xi_{T-1}^{u} S_{T-1}-K\right)^{+}}{\hat{r}\left(\xi_{T-1}^{u}-\xi_{T-1}^{d}\right)} .
\end{gathered}
$$

Now we calculate the wealth of portfolio at time $T-1$

$$
\begin{aligned}
& V_{T-1}(\phi)=\alpha_{T-1} S_{T-1}+\beta_{T-1} \\
& =\frac{\left(\xi_{T-1}^{u} S_{T-1}-K\right)^{+}-\left(\xi_{T-1}^{d} S_{T-1}-K\right)^{+}}{\left(\xi_{T-1}^{u}-\xi_{T-1}^{d}\right)}+\frac{\xi_{T-1}^{u}\left(\xi_{T-1}^{d} S_{T-1}-K\right)^{+}-\xi_{T-1}^{d}\left(\xi_{T-1}^{u} S_{T-1}-K\right)^{+}}{\hat{r}\left(\xi_{T-1}^{u}-\xi_{T-1}^{d}\right)} \\
& =\frac{\left(\xi_{T-1}^{u} S_{T-1}-K\right)^{+}\left(\hat{r}-\xi_{T-1}^{d}\right)}{\hat{r}\left(\xi_{T-1}^{u}-\xi_{T-1}^{d}\right)}-\frac{\left(\xi_{T-1}^{d} S_{T-1}-K\right)^{+}\left(\hat{r}-\xi_{T-1}^{u}\right)}{\hat{r}\left(\xi_{T-1}^{u}-\xi_{T-1}^{d}\right)}
\end{aligned}
$$

We denote

$$
p_{T-1}:=\frac{\hat{r}-\xi_{T-1}^{d}}{\xi_{T-1}^{u}-\xi_{T-1}^{d}}, \quad q_{T-1}:=\frac{\xi_{T-1}^{u}-\hat{r}}{\xi_{T-1}^{u}-\xi_{T-1}^{d}},
$$

where $q_{T-1}=1-p_{T-1}$. Herefrom it follows that

$$
V_{T-1}(\phi)=\frac{1}{\hat{r}}\left[\left(\xi_{T-1}^{u} S_{T-1}-K\right)^{+} p_{T-1}+\left(\xi_{T-1}^{d} S_{T-1}-K\right)^{+} q_{T-1}\right] .
$$




\subsection{Generalized CRR Arbitrage Option Pricing}

The necessary and sufficient conditions for the absence of arbitrage in the market are presented as

$$
\mathbb{P}\left\{\xi_{t}^{d}<1+r<\xi_{t}^{u}\right\}=1, \quad \forall \quad t \leq T .
$$

Under these assumptions the value of portfolio at time $T-1$ equals to the option payoff $C_{T-1}$

$$
C_{T-1}=\frac{1}{\hat{r}}\left[\left(\xi_{T-1}^{u} S_{T-1}-K\right)^{+} p_{T-1}+\left(\xi_{T-1}^{d} S_{T-1}-K\right)^{+} q_{T-1}\right] .
$$

Consider now the time period $[T-2, T-1]$. On this step we search for a portfolio $\phi_{T-2}=\left(\alpha_{T-2}, \beta_{T-2}\right)$ which is created at time $T-2$ in such a way that its wealth at time $T-1$ replicates option value $C_{T-1}$, that is

$$
V_{T-1}(\phi)=C_{T-1}=\alpha_{T-2} S_{T-1}+\beta_{T-2} \hat{r}
$$

Since the dynamic strategy $\phi$ constructed in this way has the self-financing property at time $T-1$, then

$$
\alpha_{T-2} S_{T-1}+\beta_{T-2} \hat{r}=\alpha_{T-1} S_{T-1}+\beta_{T-1} .
$$

Using this property and (1.3.3) we write the system of equations

$$
\left\{\begin{array}{c}
\alpha_{T-2} \xi_{T-2}^{u} S_{T-2}+\beta_{T-2} \hat{r}=C_{T-1}^{\xi_{T-2}^{u}} \\
\alpha_{T-2} \xi_{T-2}^{d} S_{T-2}+\beta_{T-2} \hat{r}=C_{T-1}^{\xi_{T-2}^{d}}
\end{array}\right.
$$

where by virtue of (1.3.2)

$$
\begin{aligned}
& C_{T-1}^{\xi_{T-2}^{u}}=\frac{1}{\hat{r}}\left[\left(\xi_{T-1}^{u} \xi_{T-2}^{u} S_{T-1}-K\right)^{+} p_{T-1}+\left(\xi_{T-1}^{d} \xi_{T-2}^{u} S_{T-1}-K\right)^{+} q_{T-1}\right], \\
& C_{T-1}^{\xi_{T-2}^{d}}=\frac{1}{\hat{r}}\left[\left(\xi_{T-1}^{u} \xi_{T-2}^{d} S_{T-1}-K\right)^{+} p_{T-1}+\left(\xi_{T-1}^{d} \xi_{T-2}^{d} S_{T-1}-K\right)^{+} q_{T-1}\right] .
\end{aligned}
$$

The solutions of (1.3.4) are given by

$$
\begin{gathered}
\alpha_{T-2}=\frac{C_{T-1}^{\xi_{T-2}^{u}}-C_{T-1}^{\xi_{T-2}^{d}}}{S_{T-2}\left(\xi_{T-2}^{u}-\xi_{T-2}^{d}\right)}, \\
\beta_{T-2}=\frac{\xi_{T-2}^{u} C_{T-1}^{\xi_{T-2}^{d}}-\xi_{T-2}^{d} C_{T-1}^{\xi_{T-2}^{u}}}{\hat{r}\left(\xi_{T-2}^{u}-\xi_{T-2}^{d}\right)} .
\end{gathered}
$$




\subsection{Generalized CRR Arbitrage Option Pricing}

Therefore, the wealth of portfolio $V_{T-2}(\phi)$ can be calculated as

$$
\begin{aligned}
& V_{T-2}(\phi)=\alpha_{T-2} S_{T-2}+\beta_{T-2}=\frac{C_{T-1}^{\xi_{T-2}^{u}-C_{T-1}^{\xi_{T-2}^{d}}}}{\xi_{T-2}^{u}-\xi_{T-2}^{d}}+\frac{\xi_{T-2}^{u} C_{T-1}^{\xi_{T-2}^{d}-\xi_{T-2}^{d}} C_{T-1}^{\xi_{T-2}^{u}}}{\hat{r}\left(\xi_{T-2}^{u}-\xi_{T-2}^{d}\right)} \\
& =\frac{C_{T-1}^{\xi_{T-2}^{u}}\left(\hat{r}-\xi_{T-2}^{d}\right)}{\hat{r}\left(\xi_{T-2}^{u}-\xi_{T-2}^{d}\right)}+\frac{C_{T-1}^{\xi_{T-2}^{d}}\left(\xi_{T-2}^{u}-\hat{r}\right)}{\hat{r}\left(\xi_{T-2}^{u}-\xi_{T-2}^{d}\right)}=\frac{1}{\hat{r}}\left[C_{T-1}^{\xi_{T-2}^{u}} p_{T-2}+C_{T-1}^{\xi_{T-2}^{d}} q_{T-2}\right],
\end{aligned}
$$

where we denote as

$$
p_{T-2}:=\frac{\hat{r}-\xi_{T-2}^{d}}{\xi_{T-2}^{u}-\xi_{T-2}^{d}}, \quad q_{T-2}:=\frac{\xi_{T-2}^{u}-\hat{r}}{\xi_{T-2}^{u}-\xi_{T-2}^{d}} .
$$

Having substituted the expressions for $C_{T-1}^{\xi_{T-2}^{u}}$ and $C_{T-1}^{\xi_{T-2}^{d}}$ we obtain

$$
\begin{aligned}
& V_{T-2}(\phi)=\frac{1}{\hat{r}^{2}}\left[\left(\xi_{T-1}^{u} \xi_{T-2}^{u} S_{T-2}-K\right)^{+} p_{T-1} p_{T-2}\right. \\
& +\left(\xi_{T-1}^{d} \xi_{T-2}^{u} S_{T-2}-K\right)^{+} q_{T-1} p_{T-2}+\left(\xi_{T-1}^{u} \xi_{T-2}^{d} S_{T-2}-K\right)^{+} p_{T-1} q_{T-2} \\
& \left.+\left(\xi_{T-1}^{d} \xi_{T-2}^{d} S_{T-2}-K\right)^{+} q_{T-1} q_{T-2}\right] .
\end{aligned}
$$

The wealth of portfolio $V_{T-2}(\phi)$ at time $T-2$ replicates the arbitrage price of the call option at time $T-2$, i.e. $C_{T-2}=V_{T-2}(\phi)$ and

$$
\begin{aligned}
& C_{T-2}=\frac{1}{\hat{r}^{2}}\left[\left(\xi_{T-1}^{u} \xi_{T-2}^{u} S_{T-2}-K\right)^{+} p_{T-1} p_{T-2}\right. \\
& +\left(\xi_{T-1}^{d} \xi_{T-2}^{u} S_{T-2}-K\right)^{+} q_{T-1} p_{T-2}+\left(\xi_{T-1}^{u} \xi_{T-2}^{d} S_{T-2}-K\right)^{+} p_{T-1} q_{T-2} \\
& \left.+\left(\xi_{T-1}^{d} \xi_{T-2}^{d} S_{T-2}-K\right)^{+} q_{T-1} q_{T-2}\right] .
\end{aligned}
$$

Implementing the same procedure for time period $[T-3, T-2]$ we derive the formula for payoff of the option at time $T-3, C_{T-3}$. It is clear that if this procedure is repeated desired number of times, then it is possible to completely determine the arbitrage option price at any time $t=T-m, 0 \leq m \leq T$, as well as the unique trading strategy $\phi$ that replicates the option.

By construction of the replicating strategy, for any fixed time $t$ random variables $\alpha_{t}$ and $\beta_{t}$ which define the portfolio at time $t$, as well as the wealth $V_{t}(\phi)$ of this portfolio, are measurable with respect to the $\sigma$-field $\mathcal{F}_{t}^{S}$. 


\subsection{Random sets. Auxiliary results}

First, introduce some notations. We denote the set $\Gamma_{m}:=\{1, \ldots, m\}$. Moreover, for any fixed $m \in \mathbb{N}$ and $j \in \Gamma_{m}$ we define a set

$$
I_{j, m}(x)=\left\{J \subset \Gamma_{m},|J|=j: \quad x \prod_{k \in J} \xi_{T-k}^{u} \prod_{k \in \bar{J}} \xi_{T-k}^{d}>K\right\} .
$$

Here $|J|$ stands for the cardinality of the set $J, \bar{J}$ stands for the complement of the set $J$.

The set $I_{j, m}(x)$ is a set of all subsets of $\Gamma_{m}$ with cardinality equal to $j$ such that the corresponding inequality holds true. Further we describe some properties of the set $I_{j, m}(x)$.

The set $I_{j, m}(x)$ is a random set, where randomness is provided by random variables $\xi_{T-k}^{u}$ and $\xi_{T-k}^{d}, k \leq T$. And for any $\omega \in \Omega$ and fixed $j \in \mathbb{N}$ the set $I_{j, m}(x)$ is either empty set $\emptyset$ or a set $I:=\{$ all $J:|J|=j\}$ of all subsets $J \subset \Gamma_{m}$ of cardinality $j$.

Define $\Delta_{m}(x)$ as

$$
\Delta_{m}(x):=\frac{1}{\hat{r}^{m}} \sum_{j=0}^{m} \sum_{J \in I_{j, m}^{p}(x)} \prod_{k \in J} p_{T-k} \prod_{k \notin J} q_{T-k}\left(x \prod_{k \in J} \xi_{T-k}^{u} \prod_{k \notin J} \xi_{T-k}^{d}-K\right)
$$

where $I_{j, m}^{p}(x) \subseteq I_{j, m}(x)$ is a subset (some part) of $I_{j, m}(x)$.

Define $a_{m}(x)$ as

$$
a_{m}(x):=\inf \left\{j: I_{j, m}(x) \neq \emptyset\right\} .
$$

It follows that for any $j \geq a_{m}(x)$ and for any set $J$ of cardinality $j$ the inequality

$$
x \prod_{k \in J} \xi_{T-k}^{u} \prod_{k \in \bar{J}} \xi_{T-k}^{d}>K
$$

holds true with probability one.

From now and later in this section the sign " $\cong$ will stand for isomorphism of sets. 
Lemma 1.4.1. For $j \in \Gamma_{m}$ the following sets

$$
I_{j, m}\left(\xi_{T-m-1}^{u} S_{T-m-1}\right) \bigcup I_{j, m}\left(\xi_{T-m-1}^{d} S_{T-m-1}\right) \cong I_{j, m+1}\left(S_{T-m-1}\right)
$$

are isomorphic almost everywhere.

Proof. The inclusion

$$
I_{j, m}\left(\xi_{T-m-1}^{u} S_{T-m-1}\right) \bigcup I_{j, m}\left(\xi_{T-m-1}^{d} S_{T-m-1}\right) \subseteq I_{j, m+1}\left(S_{T-m-1}\right)
$$

is evident. To prove the inverse inclusion we find out that for any $j \in \Gamma_{m}$

$$
\begin{aligned}
& I_{j, m}\left(\xi_{T-m-1}^{u} S_{T-m-1}\right) \\
& =\left\{J \subset \Gamma_{m},|J|=j, j \in \Gamma_{m}: S_{T-m-1} \xi_{T-m-1}^{u} \prod_{k \in J} \xi_{T-k}^{u} \prod_{k \in \bar{J}} \xi_{T-k}^{d}>K\right\} \\
& \cong\left\{J^{*} \subset \Gamma_{m+1}, J^{*}=J \bigcup\{m+1\},\left|J^{*}\right|=j, j \in \Gamma_{m+1}:\right. \\
& \left.S_{T-m-1} \prod_{k \in J^{*}} \xi_{T-k}^{u} \prod_{k \in \bar{J}^{*}} \xi_{T-k}^{d}>K\right\}=: I_{j,(m+1)^{+}}\left(S_{T-m-1}\right)
\end{aligned}
$$

Also, for $j \in \Gamma_{m}$ we show that

$$
\begin{aligned}
& I_{j, m}\left(\xi_{T-m-1}^{d} S_{T-m-1}\right) \\
& =\left\{J \subset \Gamma_{m},|J|=j, j \in \Gamma_{m}: S_{T-m-1} \xi_{T-m-1}^{d} \prod_{k \in J} \xi_{T-k}^{u} \prod_{k \in \bar{J}} \xi_{T-k}^{d}>K\right\} \\
& \cong\left\{J^{*} \subset \Gamma_{m+1},\left|J^{*}\right|=j, j \in \Gamma_{m+1}, m+1 \notin J^{*}:\right. \\
& \left.S_{T-m-1} \prod_{k \in J^{*}} \xi_{T-k}^{u} \prod_{k \in \bar{J}^{*}} \xi_{T-k}^{d}>K\right\}=: I_{j,(m+1)^{-}}\left(S_{T-m-1}\right) .
\end{aligned}
$$

This means that for any $j \in \Gamma_{m+1}$

$$
\begin{aligned}
I_{j, m}\left(\xi_{T-m-1}^{u} S_{T-m-1}\right) \cup I_{j, m} & \left(\xi_{T-m-1}^{d} S_{T-m-1}\right) \\
& \cong I_{j,(m+1)^{+}}\left(S_{T-m-1}\right) \bigcup I_{j,(m+1)^{-}}\left(S_{T-m-1}\right) .
\end{aligned}
$$

Consider a set $J_{1} \in I_{j, m+1}\left(S_{T-m-1}\right)$. For $j \in \Gamma_{m}$ we have two possibilities: 


\subsection{Generalized CRR Option Pricing Formula}

(1) $m+1 \notin J_{1}, \quad$ then $\quad J_{1} \in I_{j,(m+1)^{-}}\left(S_{T-m-1}\right)$

(2) $m+1 \in J_{1}$, then $J_{1} \in I_{j,(m+1)^{+}}\left(S_{T-m-1}\right)$.

For $j=m+1$ we have the only possibility when

$$
m+1 \in J_{1} \quad \text { and therefore } \quad J_{1} \in I_{j,(m+1)^{+}}\left(S_{T-m-1}\right) .
$$

It implies that $J_{1} \in I_{j,(m+1)^{+}}\left(S_{T-m-1}\right) \bigcup I_{j,(m+1)^{-}}\left(S_{T-m-1}\right)$. The lemma is proved.

To shorten the lengthy expressions we introduce the following notations

$$
\begin{aligned}
\bar{P}\left(J^{(j)}, T\right) & :=\prod_{k \in J} \bar{p}_{T-k} \prod_{k \notin J} \bar{q}_{T-k}, \\
P\left(J^{(j)}, T\right) & :=\prod_{k \in J} p_{T-k} \prod_{k \notin J} q_{T-k}, \\
\Xi^{u, d}\left(J^{(j)}, T\right) & :=\prod_{k \in J} \xi_{T-k}^{u} \prod_{k \notin J} \xi_{T-k}^{d} .
\end{aligned}
$$

We have to notice that $P\left(J^{(j)}, T\right)$ and $\bar{P}\left(J^{(j)}, T\right)$ are random variables where randomness is provided by $\xi_{t}^{u}$ and $\xi_{t}^{d}$ for all $t \leq T$.

\subsection{Generalized CRR Option Pricing Formula}

In this section we derive CRR type option price formula provided that the underlying stock price process follows generalized CRR stock price model defined in definition (1.1.1). Financial security market $\mathcal{M}$ is assumed to be complete and arbitrage free. Then the arbitrage generalized CRR option price is established by the following result.

Proposition 1.5.1. The arbitrage price of a European call option at time $t=T-m$ is given by the following formula

$$
C_{T-m}=S_{T-m} \sum_{j=0}^{m}\left(\sum_{J \in I_{j, m}\left(S_{T-m}\right)} \bar{P}\left(J^{(j)}, T\right)-\frac{K}{\hat{r}^{m}} \sum_{J \in I_{j, m}\left(S_{T-m}\right)} P\left(J^{(j)}, T\right)\right)
$$


for $m=0, \ldots, T$, where

$$
p_{T-k}:=\frac{\hat{r}-\xi_{T-k}^{d}}{\xi_{T-k}^{u}-\xi_{T-k}^{d}}, \quad \bar{p}_{T-k}:=\frac{\xi_{T-k}^{u}}{\hat{r}} p_{T-k}, \quad q_{T-k}=1-p_{T-k}, \quad \bar{q}_{T-k}=1-\bar{p} .
$$

At time $t=T-m-1$ the unique replicating strategy $\phi$ is defined as

$$
\begin{gathered}
\alpha_{T-m-1}=\sum_{j=0}^{m} \sum_{J \in I_{j, m}\left(\xi_{T-m-1}^{d} S_{T-m-1}\right)} \bar{P}\left(J^{(j)}, T\right)+\frac{\delta \Delta_{m}\left(\xi_{T-m-1}^{u} S_{T-m-1}\right)}{S_{T-m-1}\left(\xi_{T-m-1}^{u}-\xi_{T-m-1}^{d}\right)}, \\
\beta_{T-m-1}=-\frac{K}{\hat{r}^{m+1}} \sum_{j=0}^{m} \sum_{J \in I_{j, m}\left(\xi_{T-m-1}^{d} S_{T-m-1}\right)} P\left(J^{(j)}, T\right)-\frac{\delta \xi_{T-m-1}^{d} \Delta_{m}\left(\xi_{T-m-1}^{u} S_{T-m-1}\right)}{\hat{r}\left(\xi_{T-m-1}^{u}-\xi_{T-m-1}^{d}\right)},
\end{gathered}
$$

where $I_{j, m}^{p}\left(\xi_{T-m-1}^{u} S_{T-m-1}\right)=I_{j, m}\left(\xi_{T-m-1}^{u} S_{T-m-1}\right) \backslash I_{j, m}\left(\xi_{T-m-1}^{d} S_{T-m-1}\right)$

and $\delta=0$ if $I_{j, m}^{p}\left(\xi_{T-m-1}^{u} S_{T-m-1}\right)=\emptyset$ (otherwise $\left.\delta=1\right)$.

Proof. The right hand side of (1.5.1) due to notations (1.4.2), (1.4.3), (1.4.4) is equivalent to

$$
\begin{aligned}
& \frac{S_{T-m}}{\hat{r}^{m}} \sum_{j=0}^{m} \sum_{J \in I_{j, m}\left(S_{T-m}\right)} P\left(J^{(j)}, T\right) \Xi^{u, d}\left(J^{(j)}, T\right)-\frac{K}{\hat{r}^{m}} \sum_{j=0}^{m} \sum_{J \in I_{j, m}\left(S_{T-m}\right)} P\left(J^{(j)}, T\right) \\
& =\frac{1}{\hat{r}^{m}} \sum_{j=0}^{m} \sum_{J \in I_{j, m}\left(S_{T-m}\right)} P\left(J^{(j)}, T\right)\left(S_{T-m} \Xi^{u, d}\left(J^{(j)}, T\right)-K\right) \\
& =\frac{1}{\hat{r}^{m}} \sum_{j=0}^{m} \sum_{J \in I_{j, m}\left(S_{T-m}\right)} P\left(J^{(j)}, T\right)\left(S_{T-m} \Xi^{u, d}\left(J^{(j)}, T\right)-K\right)^{+} .
\end{aligned}
$$

To prove (1.5.1) we use the method of mathematical induction with respect to number of steps back $m$. For the base of induction, $m=0$, the option price equals

$$
C_{T}=\left(S_{T}-K\right)^{+},
$$

which has been a payoff of the option at expiry date $T$. Assume that $C_{T-m}$ is the arbitrage price of European call option at time $T-m$ for which the formula (1.5.1) holds true. We have to select a portfolio $\phi_{T-m-1}=\left(\alpha_{T-m-1}, \beta_{T-m-1}\right)$ for the time period $[T-m-1, T-m)$ (i.e. established at time $T-m-1$ ) in such 


\subsection{Generalized CRR Option Pricing Formula}

a way that the portfolio's wealth at time $t=T-m$ replicates the value $C_{T-m}$ of the option. Formally, the wealth of the portfolio $\left(\alpha_{T-m-1}, \beta_{T-m-1}\right)$ has to satisfy the following relation.

$$
C_{T-m}=V_{T-m}(\phi), \quad V_{T-m}=\alpha_{T-m-1} S_{T-m}+\beta_{T-m-1} \hat{r},
$$

so that we can derive the system of equations

$$
\left\{\begin{array}{l}
\alpha_{T-m-1} \xi_{T-m-1}^{u} S_{T-m-1}+\beta_{T-m-1} \hat{r}=C_{T-m}^{\xi_{T-m-1}^{u}} \\
\alpha_{T-m-1} \xi_{T-m-1}^{d} S_{T-m-1}+\beta_{T-m-1} \hat{r}=C_{T-m}^{\xi_{T-m-1}^{d}},
\end{array}\right.
$$

where

$$
\begin{aligned}
& C_{T-m}^{\xi_{T-m-1}^{u}}=\frac{1}{\hat{r}^{m}} \sum_{j=0}^{m} \sum_{J \in I_{j, m}\left(\xi_{T-m-1}^{u} S_{T-m}\right)} P\left(J^{(j)}, T\right)\left(\xi_{T-m-1}^{u} S_{T-m-1} \Xi^{u, d}\left(J^{(j)}, T\right)-K\right), \\
& C_{T-m}^{\xi_{T-m-1}^{d}}=\frac{1}{\hat{r}^{m}} \sum_{j=0}^{m} \sum_{J \in I_{j, m}\left(\xi_{T-m-1}^{d} S_{T-m}\right)} P\left(J^{(j)}, T\right)\left(\xi_{T-m-1}^{d} S_{T-m-1} \Xi^{u, d}\left(J^{(j)}, T\right)-K\right) .
\end{aligned}
$$

Since $\xi_{t}^{d}<\xi_{t}^{u}$ for any $t \leq T$ and $d<u$ with probability one, it is clear, that

$$
I_{j, m}\left(\xi_{T-m-1}^{d} S_{T-m-1}\right) \subseteq I_{j, m}\left(\xi_{T-m-1}^{u} S_{T-m-1}\right) .
$$

The number of shares held during the period $[T-m-1, T-m)$ is given by

$$
\begin{aligned}
& \alpha_{T-m-1}=\frac{C_{T-m}^{\xi_{T-m-1}^{u}-C_{T-m}^{\xi_{T-m-1}^{d}}}}{S_{T-m-1}\left(\xi_{T-m-1}^{u}-\xi_{T-m-1}^{d}\right)}=\frac{1}{\hat{r} S_{T-m-1}\left(\xi_{T-m-1}^{u}-\xi_{T-m-1}^{d}\right)} \\
& \times\left[\sum_{j=0}^{m} \sum_{J \in I_{j, m}^{p}\left(\xi_{T-m-1}^{u} S_{T-m-1}\right)} \xi_{T-m-1}^{u} S_{T-m-1} P\left(J^{(j)}, T\right) \Xi^{u, d}\left(J^{(j)}, T\right)-K\right) \\
& +\sum_{j=0}^{m} \sum_{J \in I_{j, m}\left(\xi_{T-m-1}^{d} S_{T-m-1}\right)} P\left(J^{(j)}, T\right)\left(\xi_{T-m-1}^{u} S_{T-m-1} \Xi^{u, d}\left(J^{(j)}, T\right)-K\right) \\
& \left.-\sum_{j=0}^{m} \sum_{J \in I_{j, m}\left(\xi_{T-m-1}^{d} S_{T-m-1}\right)} P\left(J^{j}, T\right)\left(\xi_{T-m-1}^{d} S_{T-m-1} \Xi^{u, d}\left(J^{(j)}, T\right)-K\right)\right] \\
& =\frac{\Delta_{m}\left(\xi_{T-m-1}^{u} S_{T-m-1}\right)}{S_{T-m-1}\left(\xi_{T-m-1}^{u}-\xi_{T-m-1}^{d}\right)}+\frac{1}{\hat{r}^{m}} \sum_{j=0}^{m} \sum_{J \in I_{j, m}\left(\xi_{T-m-1}^{d} S_{T-m-1}\right)} P\left(J^{(j)}, T\right) \Xi^{u, d}\left(J^{j}, T\right)
\end{aligned}
$$




$$
=\frac{\Delta_{m}\left(\xi_{T-m-1}^{u} S_{T-m-1}\right)}{S_{T-m-1}\left(\xi_{T-m-1}^{u}-\xi_{T-m-1}^{d}\right)}+\sum_{j=0}^{m} \sum_{J \in I_{j, m}\left(\xi_{T-m-1}^{d} S_{T-m-1}\right)} \bar{P}\left(J^{(j)}, T\right) .
$$

The dollar investment at time $t=T-m-1$ is equal to

$$
\begin{aligned}
& \beta_{T-m-1}=\frac{\xi_{T-m-1}^{u} C_{T-m}^{\xi_{T-m-1}^{d}-\xi_{T-m-1}^{d}} C_{T-m}^{\xi_{T-m-1}^{u}}}{\hat{r}\left(\xi_{T-m-1}^{u}-\xi_{T-m-1}^{d}\right)}=\frac{1}{\hat{r}\left(\xi_{T-m-1}^{u}-\xi_{T-m-1}^{d}\right)} \\
& \times\left[\frac{\xi_{T-m-1}^{u}}{\hat{r}^{m}} \sum_{j=0}^{m} \sum_{J \in I_{j, m}\left(\xi_{T-m-1}^{d} S_{T-m-1}\right)} P\left(J^{(j)}, T\right)\left(\xi_{T-m-1}^{d} S_{T-m-1} \Xi^{u, d}\left(J^{(j)}, T\right)-K\right)\right. \\
& -\frac{\xi_{T-m-1}^{d}}{\hat{r}^{m}} \sum_{j=0}^{m} \sum_{J \in I_{j, m}^{p}\left(\xi_{T-m-1}^{u} S_{T-m-1}\right)} P\left(J^{(j)}, T\right)\left(\xi_{T-m-1}^{u} S_{T-m-1}^{u} \Xi^{u, d}\left(J^{(j)}, T\right)-K\right) \\
& \left.-\frac{\xi_{T-m-1}^{d}}{\hat{r}^{m}} \sum_{j=0}^{m} \sum_{J \in I_{j, m}\left(\xi_{T-m-1}^{d} S_{T-m-1}\right)}^{m} P\left(J^{(j)}, T\right)\left(\xi_{T-m-1}^{u} S_{T-m-1} \Xi^{u, d}\left(J^{(j)}, T\right)-K\right)\right] \\
& =-\frac{\xi_{T-m-1}^{d} \Delta_{m}\left(\xi_{T-m-1}^{u} S_{T-m-1}\right)}{\hat{r}\left(\xi_{T-m-1}^{u}-\xi_{T-m-1}^{d}\right)}-\frac{K}{\hat{r}^{m+1}} \sum_{j=0}^{m} \sum_{J \in I_{j, m}\left(\xi_{T-m-1}^{d} S_{T-m-1}\right)}^{m} P\left(J^{(j)}, T\right) .
\end{aligned}
$$

By substituting $\alpha_{T-m-1}$ and $\beta_{T-m-1}$ for the obtained expressions, the wealth of portfolio at time $t=T-m-1$ equals to

$$
\begin{aligned}
& C_{T-m-1}=\alpha_{T-m-1} S_{T-m-1}+\beta_{T-m-1}=\frac{1}{\hat{r}}\left(C_{T-m}^{\xi_{T-m-1}^{u}} p_{T-m-1}+C_{T-m}^{\xi_{T-m-1}^{d}} q_{T-m-1}\right) \\
& \left.=\frac{1}{\hat{r}^{m+1}} \sum_{j=0}^{m} \sum_{J \in I_{j,(m+1)+}} S_{T-m-1}\right) \\
& \quad+\frac{1}{\hat{r}^{m+1}} \sum_{j=0}^{m} \sum_{J \in I_{j,(m+1)-}\left(S_{T-m-1}\right)} P\left(J^{(j)}, T\right)\left(S_{T-m-1} \Xi^{u, d}\left(J^{(j)}, T\right)-K\right)\left(S_{T-m-1} \Xi^{u, d}\left(J^{(j)}, T\right)-K\right) \\
& =\frac{1}{\hat{r}^{m+1}} \sum_{j=0}^{m+1} \sum_{J \in I_{j, m+1}\left(S_{T-m-1}\right)} P\left(J^{(j)}, T\right)\left(S_{T-m-1} \Xi^{u, d}\left(J^{(j)}, T\right)-K\right)^{+} .
\end{aligned}
$$

The proposition is proved.

Remark 1.5.2. One should notice that the option price $C_{T-m}$ given by 


\subsection{Generalized CRR Option Pricing Formula}

formula (1.5.1) now has two sources of randomness, first it's dependent on the random sequence $X_{T-m}, \ldots, X_{T}$ and the another type of randomness is provided by $S_{T-m}$, stock price at the moment $t=T-m$. By other words $C_{T-m}$ is a function of random variables $X_{T-m}, \ldots, X_{T}, S_{T-m}$.

$$
C_{T-m}=C_{T-m}\left(S_{T-m}, X_{T-m}, \ldots, X_{T}\right)
$$

In the setting of classical Cox-Ross-Rubinstein model the condition

$$
\mathbf{P}\left\{X_{T-m}=\ldots=X_{T}=1\right\}=1
$$

is satisfied and under this condition one has the classical Cox-Ross-Ruinstein [20] binomial option price. The corresponding definitions of the set $I_{j, m}(x)$, which will not be random set anymore under the condition $\mathbf{P}\left\{X_{T-m}=\ldots=X_{T}=1\right\}=1$, of the values $\Delta_{m}(x)$ and $a_{m}(x)$ look much simpler. 


\section{Chapter 2}

\section{Conditional Generalized Cox-Ross-Rubinstein Model}

In this chapter we consider the generalized CRR option price (1.5.1) provided that some assumptions about the distribution of the family of random variables $\left(X_{k}\right)_{k=0}^{m}, \quad m=0, \ldots, T$ have been made. We concentrate on some specific case of the domain of possible values of $\left(X_{k}\right)_{k=0}^{m}, \quad m=0, \ldots, T$. Under these particular assumptions the generalized CRR option price has been derived. It turned out that the terminal formula is expressed via multinomial coefficients, therefore this discrete-time option valuation formula will be referred to as multinomial CRR model.

\subsection{Conditional CRR Option Price}

We work with a finite probability space $(\Omega, \mathcal{F}, \mathbb{P})$ with a finite number of points $\omega$, each with positive probability $\mathbb{P}\{\omega\}>0$. Let $\left(X_{t}\right)_{t \leq T}$ is a family of random functions of discrete argument $t \in\{0, \ldots, T\}$. We assume that the corresponding family of measures on the real line $\left(\mathcal{P}_{t}\right)_{0 \leq t \leq T}$ is known. More precisely, the family of these measures is described as follow.

$$
\begin{aligned}
& \mathcal{P}_{t_{1}}(A)=\mathbb{P}\left\{X_{t_{1}} \in A\right\}, \quad A \in \mathbb{R}^{1}, \\
& \mathcal{P}_{t_{1}, t_{2}}(A)=\mathbb{P}\left\{\left(X_{t_{1}}, X_{t_{2}}\right) \in A\right\}, \quad A \in \mathbb{R}^{2}, \\
& \quad \ldots \ldots, \quad A \in \mathbb{R}^{k} \\
& \mathcal{P}_{t_{1}, \ldots, t_{k}}(A)=\mathbb{P}\left\{\left(X_{t_{1}}, \ldots, X_{t_{k}}\right) \in A\right\}, \quad
\end{aligned}
$$




\subsection{Conditional CRR Option Price}

The family of finite-dimensional distributions satisfy the conditions of coordination, i.e.

$$
\mathcal{P}_{t_{1}, \ldots, t_{k}}(A)=\mathcal{P}_{t_{1}, \ldots, t_{k}, t_{k+1}, \ldots, t_{k+n}}\left(A \times \mathbb{R}^{n}\right), \quad \forall A \in \mathbb{R}^{k} .
$$

Then, due to Kolmogorov theorem (see [59]) the probability space $(\Omega, \mathcal{F}, \mathbb{P})$ and the stochastic process $X=\left(X_{t}\right)_{0 \leq t \leq T}$ exist and

$$
\mathbb{P}\left\{w: X_{t_{1}} \leq x_{1}, \ldots, X_{t_{k}} \leq x_{k}\right\}=F_{t_{1}, \ldots, t_{k}}\left(x_{1}, \ldots, x_{k}\right)
$$

Now we consider the mean of conditional expectation of (1.5.1), $C_{T-m}$, given random sequence $X_{T-m}, \ldots, X_{T}$.

$$
\tilde{C}_{T-m}=E\left\{E\left\{C_{T-m} \mid X_{T-m}, \ldots, X_{T}\right\}\right\}=E\left(C_{T-m}\right) .
$$

To simplify lengthy expressions we introduce the following notations, which are similar to $(1.4 .2),(1.4 .3)$. For $\forall z=\left(z_{T-m}, \ldots, z_{T}\right) \in \mathbb{R}^{m}$

$$
\begin{aligned}
& \bar{P}\left(J^{(j)}, T, z\right):=\prod_{k \in J} \bar{p}_{T-k}\left(z_{T-k}\right) \prod_{k \notin J} \bar{q}_{T-k}\left(z_{T-k}\right), \\
& P\left(J^{(j)}, T, z\right):=\prod_{k \in J} p_{T-k}\left(z_{T-k}\right) \prod_{k \notin J} q_{T-k}\left(z_{T-k}\right),
\end{aligned}
$$

where

$$
p_{T-k}\left(z_{T-k}\right):=\frac{\hat{r}-d z_{T-k}}{u z_{T-k}-d z_{T-k}}, \quad \bar{p}_{T-k}\left(z_{T-k}\right):=\frac{u z_{T-k}}{\hat{r}} p_{T-k}\left(z_{T-k}\right)
$$

and

$$
q_{T-k}\left(z_{T-k}\right)=1-p_{T-k}\left(z_{T-k}\right), \quad \bar{q}_{T-k}\left(z_{T-k}\right)=1-\bar{p}\left(z_{T-k}\right) .
$$

We also define the following quantities. For $\forall z=\left(z_{T-m}, \ldots, z_{T}\right) \in \mathbb{R}^{m}$

$$
\begin{aligned}
\tilde{C}_{T-m}^{(1)}(j) & :=\underbrace{\int \cdots \int}_{m \text { integrals }} \sum_{J \in I_{j, m}\left(S_{T-m}, z\right)} \bar{P}\left(J^{(j)}, T, z\right) d F^{X_{T-m}, \ldots, X_{T}}(z), \\
\tilde{C}_{T-m}^{(2)}(j) & :=\underbrace{\int \cdots \int}_{m \text { integrals }} \sum_{J \in I_{j, m}\left(S_{T-m}, z\right)} P\left(J^{(j)}, T, z\right) d F^{X_{T-m}, \ldots, X_{T}}(z),
\end{aligned}
$$


where $\forall z=\left(z_{T-m}, \ldots, z_{T}\right) \in \mathbb{R}^{m}$.

$$
I_{j, m}(x, z):=\left\{J \subset \Gamma_{m},|J|=j: \quad x \prod_{k \in J} u z_{T-k} \prod_{k \in \bar{J}} d z_{T-k}>K\right\}
$$

and $F^{X_{T-m}, \ldots, X_{T}}(z)=F_{T-m, \ldots, T}(z)$ is the joint distribution function of $X_{T-m}, \ldots, X_{T}$.

One should notice that $S_{T-m}$ is independent on $X_{T-m}, \ldots, X_{T}$. So, in general the following formula for option price holds true.

Proposition 2.1.1 The conditional generalized CRR option price is given by the following formula

$$
\tilde{C}_{T-m}=S_{T-m} \sum_{j=0}^{m} \tilde{C}_{T-m}^{(1)}(j)-\frac{K}{\hat{r}^{m}} \sum_{j=0}^{m} \tilde{C}_{T-m}^{(2)}(j) .
$$

Remark 2.1.2. The corresponding conditional replicating strategies can be derived from (1.5.2) and (1.5.3) in an analogous way as integrals with respect to the distributions family $\left(F_{t_{1}, \ldots, t_{k}}\right)_{k \geq 0}$.

If all finite-dimensional distributions of the random family $X_{T-m}, \ldots, X_{T}$ are known, then from (2.1.6) one can obtain specified corresponding option price. The option price in the form (2.1.6) gives a freedom to model underlying stock price so as it is liked.

However, in discrete-time approach the original stock price process was modeled by random walk and this is the easiest and most natural way to do it. The idea of random walk as a stock price process in financial markets stated by Burton Malkiel ( [39]) in 1973 means that stocks take a random and unpredictable path.

While the classical random walk is defined by the sequence of two-value independent random variables, we consider so called weighted random walk embedding the random family $(X)_{t \leq T}$ in the stock price process. Thus, we enrich the set of possible values of stock price at each time moment and thereby, the practical usage of the new model becomes more evident. 
Unlike the generalization of binomial CRR model of Rachev and Rüschendorf [53] we set conditions for stochastic process $\left(X_{t}\right)_{t \leq T}$ which has different at any time moment values of jump sizes. So, at any time moment the change of the stock price is determined by the value of stochastic process $\left(X_{t}\right)_{t \leq T}$ at that time moment.

As one can see, starting from (2.1.6) it is possible to impose different assumptions on the family of finite-dimensional distributions for $\left(X_{t}\right)_{t \leq T}$ and calculate the corresponding option price but, due to these assumptions, the calculations very often can be quite complicated.

Also, in many cases it might be that the stock price does not follow any specific distributional assumptions. The problem is how to model stock price process in order to better evaluate corresponding option prices. It should be intuitively clear that the better the modelling of underlying stock price the better the quality of option price valuation.

"The better" means that the modelled data look like original data and expose most of their features. This problem of stock price modelling is related to the issues of data approximation, specifically, financial data approximation. In the next section we propose natural and simple assumptions about possible values of random sequence $\left(X_{t}\right)_{t \leq T}$ which certainly can be interpreted as distributional assumptions as well.

\subsection{Multinomial model. Definitions}

Let $\left(X_{t}\right)_{t \leq T}$ is a sequence of independent identically distributed random variables. We define a set

$$
\mathbb{C}_{k}=\left\{c_{1}, \ldots, c_{k}\right\}, \quad k \in \mathbb{N}, \quad c_{i}>0, \quad \forall i=1, \ldots, k
$$

Random variables $X_{t}, t=1, \ldots, T$ take values in the set $\mathbb{C}_{k}$ and the events $\left\{X_{t}=c_{j}\right\}, t=1, \ldots, T, j=1, \ldots, k$ are equally likely with correspondent probabilities $p_{1}=\ldots=p_{k}=\frac{1}{k}$. 
In the setting of generalized CRR model the following condition holds true.

$$
\mathbf{P}\left\{X_{i} \in \mathbb{C}_{k}\right\}=1, \quad i=T-m, \ldots, T .
$$

Since we consider the discrete time interval $[T-m, T], m \leq T$ then it is obvious that $k<m$.

Further, we will refer to the set of possible values of $X_{T-m}, \ldots, X_{T}, \mathbb{C}_{k}$ defined above as the set of multinomiality parameters.

The following random structures take place in the context of the constructed model.

Definition 2.2.1 The sequence $N_{c_{1}}, \ldots, N_{c_{k}}$ is a sequence of random variables, which take values in the set $\Gamma_{m} \bigcup\{0\}=\{0, \ldots, m\}$ and satisfy the condition $N_{c_{1}}+\ldots+N_{c_{k}}=m$. For some fixed $\omega \in \Omega, N_{c_{i}}(\omega), i=1, \ldots, k$ denotes a number of $c_{i}$-s occurred in the sequence $X_{T-m}(\omega), \ldots, X_{T}(\omega)$.

Definition 2.2.2 The set

$$
I\left(m, j, N_{c_{1}}, \ldots, N_{c_{k}}\right):=\left\{\begin{array}{l}
\left(m_{1}, \ldots, m_{2 k}\right): m_{i} \in \Gamma_{m}, i=1, \ldots, 2 k ; \\
m_{1}+\ldots+m_{2 k}=m: m_{1}+\ldots+m_{k}=j, \\
N_{c_{1}}=m_{1}+m_{k+1}, \ldots, N_{c_{k}}=m_{k}+m_{2 k}
\end{array}\right\} .
$$

The set $I\left(m, j, N_{c_{1}}, \ldots, N_{c_{k}}\right)$ is random due to dependence on $N_{c_{1}}, \ldots, N_{c_{k}}$. Further, we will consider the summation over this set provided some conditions on the values of $N_{c_{1}}, \ldots, N_{c_{k}}$ are imposed. After that, accordingly to the conditions we will have the set $I\left(m, j, N_{c_{1}}^{*}, \ldots, N_{c_{k}}^{*}\right)$, where $N_{c_{1}}^{*}, \ldots, N_{c_{k}}^{*}$ will denote fixed constants. Virtually, one can consider a set $I\left(m, j, N_{c_{1}}, \ldots, N_{c_{k}}\right)$ as a function of the additional to $m$ and $j$ arguments $N_{c_{1}}, \ldots, N_{c_{k}}$.

The set $I\left(m, j, N_{c_{1}}, \ldots, N_{c_{k}}\right)$ is also associated with the set of all partitions $\left(J_{1}, \ldots, J_{2 k}\right)$ of the set $\Gamma_{m}=\{1, \ldots, m\}$ provided that $\left|J_{1}\right|=m_{1}, \ldots,\left|J_{2 k}\right|=m_{2 k}$. We consider that $|\emptyset|=0$. The corresponding set of partitions is given as follow. 


\section{Definition 2.2.3}

$$
D_{j, m}\left(x, c_{1}, \ldots, c_{2 k}\right):=\left\{\begin{array}{l}
\left(J_{1}, \ldots, J_{2 k}\right): \quad J_{i} \subset\{1, \ldots, m\}, \quad i=1, \ldots, 2 k \\
\sum_{i=1}^{k}\left|J_{i}\right|=j, \quad \bigcup_{i=1}^{2 k} J_{i}=\Gamma_{m}, \quad \sum_{i=1}^{2 k}\left|J_{i}\right|=m: \\
x\left(c_{1} u\right)^{\left|J_{1}\right|} \ldots\left(c_{k} u\right)^{\left|J_{k}\right|}\left(c_{1} d\right)^{\left|J_{k+1}\right|} \ldots\left(c_{k} d\right)^{\mid J_{2 k \mid}}>K .
\end{array}\right\}
$$

For any $w \in \Omega$ there is a surjective map from the set $I\left(m, j, N_{c_{1}}(w), \ldots, N_{c_{k}}(w)\right)$ to $D_{j, m}\left(x, c_{1}, \ldots, c_{2 k}\right)$, so that these two sets are epimorphic.

\section{Definition 2.2.4}

$$
I_{j, m}\left(x, N_{c_{1}}, \ldots, N_{c_{k}}\right):=\left\{J \subset \Gamma_{m}: \quad|J|=j, \quad x c_{1}^{N_{c_{1}}} \ldots c_{k}^{N_{c_{k}}} u^{j} d^{m-j}>K\right\}
$$

and

$$
a(x):=\inf \left\{j: I_{j, m}\left(x, N_{c_{1}}, \ldots, N_{c_{k}}\right) \neq \emptyset\right\} .
$$

We notice that $a(x)$ also can be defined as $a(x):=\inf \left\{j: D_{j, m}\left(x, c_{1}, \ldots, c_{k}\right) \neq \emptyset\right\}$. which is equivalent to (2.2.6).

From now and further we will use the following notations. For any $c=\left(c_{1}, \ldots, c_{k}\right) \in \mathbb{R}_{+}^{k}, p=\left(p_{1}, \ldots, p_{2 k}\right) \in \mathbb{R}^{2 k}, \bar{p}=\left(\bar{p}_{1}, \ldots, \bar{p}_{2 k}\right) \in \mathbb{R}^{2 k}$ and for $\left(m_{1}, \ldots, m_{2 k}\right)$ such that $m_{1}+\ldots+m_{2 k}=m$

$$
\begin{gathered}
\phi_{2 k}(m, c):=\left(c_{1} u\right)^{m_{1}} \ldots\left(c_{k} u\right)^{m_{k}}\left(c_{1} d\right)^{m_{k+1}} \ldots\left(c_{k} d\right)^{m_{2 k}} \\
M_{2 k}(m, \bar{p}):=\frac{m !}{m_{1} ! \ldots m_{2 k} !} \bar{p}_{1}^{m_{1}} \ldots \bar{p}_{2 k}^{m_{2 k}} \\
M_{2 k}(m, p):=\frac{m !}{m_{1} ! \ldots m_{2 k} !} p_{1}^{m_{1}} \ldots p_{2 k}^{m_{2 k}} \\
P_{1}^{2 k}(m):=p_{1}^{m_{1}} \ldots p_{2 k}^{m_{2 k}} \\
\bar{P}_{1}^{2 k}(m):=\bar{p}_{1}^{m_{1}} \ldots \bar{p}_{2 k}^{m_{2 k}}
\end{gathered}
$$

so that

$$
M_{2 k}(m, p)=\frac{m !}{m_{1} ! \ldots m_{2 k} !} P_{1}^{k}(m) P_{k+1}^{2 k}(m)
$$


Sometimes we also will use this notation instead of (2.2.8), (2.2.9).

\section{Definition 2.2.5}

$$
\Delta_{m}(x, j, c):=\frac{1}{\hat{r}^{m}} \sum_{I\left(m, j, N_{c_{1}}, \ldots, N_{c_{k}}\right)} M_{2 k}(m, \bar{p})\left(x \phi^{(2 k)}(m, c)-K\right) .
$$

\subsection{Multinomial option pricing}

Consider the conditional expectation

$$
\tilde{C}_{T-m}(c):=\tilde{C}_{T-m}\left(c_{1}, \ldots, c_{k}\right)=E\left\{C_{T-m} \mid X_{T-m}=c_{j_{1}}, \ldots, X_{T}=c_{j_{k}}\right\},
$$

where multinomiality parameters $c_{j_{i}} \in \mathbb{C}_{k}, i=1, \ldots, k$.

One should notice that the conditioning on the event $\left\{X_{T-m}=c_{j_{1}}, \ldots, X_{T}=c_{j_{k}}\right\}$ implies that numbers $N_{c_{1}}, \ldots, N_{c_{k}}$ are known. The following result for option price is obtained.

Proposition 2.3.1 If the condition

$$
\mathbf{P}\left\{X_{i} \in \mathbb{C}_{k}\right\}=1, \quad \forall i=T-m, \ldots, T
$$

is satisfied, then

$$
\begin{gathered}
\tilde{C}_{T-m}(c)=k^{m} \sum_{j=a}^{m} \sum_{I\left(m, j, N_{c_{1}}, \ldots, N_{c_{k}}\right)}\left(S_{T-m} M_{2 k}(m, \bar{p})-\frac{K}{\hat{r}^{m}} M_{2 k}(m, p)\right), \\
\forall \quad c=\left(c_{1}, \ldots, c_{k}\right) \in \mathbb{R}_{+}^{k},
\end{gathered}
$$

where $a=a\left(S_{T-m}\right)$,

$$
\begin{gathered}
p_{1}\left(c_{1}\right)=\frac{1}{k} \frac{\hat{r}-c_{1} d}{c_{1}(u-d)}, \ldots, p_{k}\left(c_{k}\right)=\frac{1}{k} \frac{\hat{r}-c_{k} d}{c_{k}(u-d)}, \\
p_{k+1}\left(c_{1}\right)=\frac{1}{k} \frac{c_{1} u-\hat{r}}{c_{1}(u-d)}, \ldots p_{2 k}\left(c_{k}\right)=\frac{1}{k} \frac{c_{k} u-\hat{r}}{c_{k}(u-d)}, \\
\bar{p}_{1}=\frac{c_{1} u}{\hat{r}} p_{1}, \ldots, \bar{p}_{k}=\frac{c_{k} u}{\hat{r}} p_{k}, \quad \bar{p}_{k+1}=\frac{c_{1} d}{\hat{r}} p_{k+1}, \ldots, \bar{p}_{2 k}=\frac{c_{k} d}{\hat{r}} p_{2 k}
\end{gathered}
$$

and

$$
p_{1}+p_{k+1}=\ldots=p_{k}+p_{2 k}=\frac{1}{k},
$$




\subsection{Multinomial option pricing}

$$
\bar{p}_{1}+\bar{p}_{k+1}=\ldots=\bar{p}_{k}+\bar{p}_{2 k}=\frac{1}{k} .
$$

At time $t=T-m-1$ the unique replicating strategy $\phi$ is given by

$$
\begin{gathered}
\alpha_{T-m-1}(c)=k^{m} \sum_{j=a^{d}}^{m} \sum_{I\left(m, j, N_{c_{1}}, \ldots, N_{c_{k}}\right)} M_{2 k}(m, \bar{p})+\frac{k^{m} \delta \Delta_{m}\left(\xi_{T-m-1}^{u} S_{T-m-1}, a^{u}, c\right)}{S_{T-m-1}\left(\xi_{T-m-1}^{u}-\xi_{T-m-1}^{d}\right)}, \\
\beta_{T-m-1}(c)=-\frac{K k^{m}}{\hat{r}^{m+1}} \sum_{j=a^{d} I\left(m, j, N_{c_{1}}, \ldots, N_{c_{k}}\right)}^{m} M_{2 k}(m, p)-\frac{k^{m} \delta \Delta_{m}\left(\xi_{T-m-1}^{u} S_{T-m-1}, a^{u}, c\right)}{\hat{r}\left(\xi_{T-m-1}^{u}-\xi_{T-m-1}^{d}\right)},
\end{gathered}
$$

where $a^{u}=a\left(\xi_{T-m-1}^{u} S_{T-m-1}\right), a^{d}=a\left(\xi_{T-m-1}^{d} S_{T-m-1}\right)$ and $\delta=0$ if $a^{d}=a^{u}$ (otherwise $\delta=1$ ).

Proof. Show that $\bar{p}_{2 k}=\frac{c_{k} d}{\hat{r}} p_{2 k}$.

$$
\begin{aligned}
\bar{p}_{2 k}= & \frac{1}{k}-\bar{p}_{k}=\frac{1}{k}-\frac{c_{k} u}{\hat{r}} p_{k}=\frac{1}{k}-\frac{1}{k} \frac{c_{k} u\left(\hat{r}-c_{k} d\right)}{\hat{r} c_{k}(u-d)}=\frac{1}{k}-\frac{u\left(\hat{r}-c_{k} d\right)}{k \hat{r}(u-d)} \\
= & \frac{c_{k} u d-\hat{r} d}{k \hat{r}(u-d)}=\frac{c_{k} d}{\hat{r}} \frac{c_{k} u-\hat{r}}{k c_{k}(u-d)}=\frac{c_{k} d}{\hat{r}}\left(1-p_{k}\right)=\frac{c_{k} d}{\hat{r}} p_{2 k} .
\end{aligned}
$$

Similarly $\bar{p}_{k}=\frac{c_{k} u}{\hat{r}} p_{k}$. Therefore, according to (2.2.7) and (2.2.10)

$$
\bar{P}_{1}^{2 k}=\frac{\phi_{2 k}(m, c)}{\hat{r}^{m}} P_{1}^{2 k}
$$

So, formula (2.3.1) is equivalent to

$$
\begin{aligned}
& \frac{k^{m}}{\hat{r}^{m}} \sum_{j=a}^{m} \sum_{I\left(m, j, N_{c_{1}}, \ldots, N_{c_{k}}\right)} M_{2 k}(m, p)\left(S_{T-m} \phi_{2 k}(m, c)-K\right) \\
= & \frac{k^{m}}{\hat{r}^{m}} \sum_{j=0}^{m} \sum_{I\left(m, j, N_{c_{1}}, \ldots, N_{c_{k}}\right)} M_{2 k}(m, p)\left(S_{T-m} \phi_{2 k}(m, c)-K\right)^{+}
\end{aligned}
$$

In order to prove (2.3.1) we use the method of mathematical induction with respect to $m$. The base of induction is when $m=0$, then we have that

$$
\tilde{C}_{T}=\left(S_{T}-K\right)^{+}
$$

which has been a payoff of the option at expiry time $T$. Assume now that for any $c=\left(c_{1}, \ldots, c_{k}\right) \in \mathbb{R}_{+}^{k}, \quad \tilde{C}_{T-m}(c)$ is the arbitrage price of the option at time $T-m$ 


\subsection{Multinomial option pricing}

which satisfy the formula (2.3.1). Portfolio $\phi_{T-m-1}(c)=\left(\alpha_{T-m-1}(c), \beta_{T-m-1}(c)\right)$ for the period $[T-m-1, T-m)$ has to be constructed in such a way that the portfolio's wealth at the end of the last period $t=T-m$ replicates option value $\tilde{C}_{T-m}(c)$; that is

$$
V_{T-m}(c)=\alpha_{T-m-1}(c) S_{T-m}+\beta_{T-m-1}(c) \hat{r} \quad \text { and } \quad V_{T-m}(c)=\tilde{C}_{T-m}(c),
$$

which is in turn allows us to obtain the following system of equations

$$
\left\{\begin{array}{l}
\alpha_{T-m-1}(c) \xi_{T-m-1}^{u} S_{T-m-1}+\beta_{T-m-1}(c) \hat{r}=\tilde{C}_{T-m}^{\xi_{T-m-1}^{u}} \\
\alpha_{T-m-1}(c) \xi_{T-m-1}^{d} S_{T-m-1}+\beta_{T-m-1}(c) \hat{r}=\tilde{C}_{T-m}^{\xi_{T-m-1}^{d}},
\end{array}\right.
$$

where

$$
\xi_{T-m-1}^{u}= \begin{cases}c_{1} u, & \mathbf{P}\left\{X_{T-m-1}=c_{1}\right\}, \\ \vdots & \\ c_{k} u, & \mathbf{P}\left\{X_{T-m-1}=c_{k}\right\},\end{cases}
$$

and

$$
\xi_{T-m-1}^{d}= \begin{cases}c_{1} d, & \mathbf{P}\left\{X_{T-m-1}=c_{1}\right\} \\ \vdots & \\ c_{k} d, & \mathbf{P}\left\{X_{T-m-1}=c_{k}\right\} .\end{cases}
$$

One should notice that

$$
\begin{aligned}
& \tilde{C}_{T-m}^{\xi_{T-m-1}^{u}(c)}=\frac{k^{m}}{\hat{r}^{m}} \sum_{j=a^{u}}^{m} \sum_{I\left(m, j, N_{c_{1}}, \ldots, N_{c_{k}}\right)} M_{2 k}(m, p)\left(\xi_{T-m-1}^{u} S_{T-m-1} \phi_{2 k}(m, c)-K\right), \\
& \tilde{C}_{T-m}^{\xi_{T-m-1}^{d}(c)}=\frac{k^{m}}{\hat{r}^{m}} \sum_{j=a^{d}}^{m} \sum_{I\left(m, j, N_{c_{1}}, \ldots, N_{c_{k}}\right)} M_{2 k}(m, p)\left(\xi_{T-m-1}^{d} S_{T-m-1} \phi_{2 k}(m, c)-K\right) .
\end{aligned}
$$

Now we can calculate the number of shares in the portfolio at time $t=T-m-1$.

For $c=\left(c_{1}, \ldots, c_{k}\right)$

$$
\begin{gathered}
\alpha_{T-m-1}(c)=\frac{\tilde{C}_{T-m}^{\xi_{T-m-1}^{u}}(c)-\tilde{C}_{T-m}^{\xi_{T-m-1}^{d}}(c)}{S_{T-m-1}\left(\xi_{T-m-1}^{u}-\xi_{T-m-1}^{d}\right)}=\frac{k^{m}}{\hat{r}^{m} S_{T-m-1}\left(\xi_{T-m-1}^{u}-\xi_{T-m-1}^{d}\right)} \\
{\left[\sum_{I\left(m, a^{u}, N_{c_{1}}, \ldots, N_{c_{k}}\right)} M_{2 k}(m, p)\left(\xi_{T-m-1}^{u} S_{T-m-1} \phi_{2 k}(m, c)-K\right)\right.}
\end{gathered}
$$




\subsection{Multinomial option pricing}

$$
\begin{gathered}
\quad+\sum_{j=a^{d}}^{m} \sum_{I\left(m, j, N_{c_{1}}, \ldots, N_{c_{k}}\right)} M_{2 k}(m, p)\left(\xi_{T-m-1}^{u} S_{T-m-1} \phi_{2 k}(m, c)-K\right) \\
\left.\quad-\sum_{j=a^{d}}^{m} \sum_{I\left(m, j, N_{c_{1}}, \ldots, N_{c_{k}}\right)} M_{2 k}(m, p)\left(\xi_{T-m-1}^{d} S_{T-m-1} \phi_{2 k}(m, c)-K\right)\right] \\
=\frac{k^{m} \Delta_{m}\left(\xi_{T-m-1}^{u} S_{T-m-1}, a^{u}, c\right)}{S_{T-m-1}\left(\xi_{T-m-1}^{u}-\xi_{T-m-1}^{d}\right)}+\frac{k^{m}}{\hat{r}^{m}} \sum_{j=a^{d}}^{m} \sum_{I\left(m, j, N_{c_{1}}, \ldots, N_{c_{k}}\right)} M_{2 k}(m, p) \phi_{2 k}(m, c) \\
=\frac{k^{m} \Delta_{m}\left(\xi_{T-m-1}^{u} S_{T-m-1}, a^{u}, c\right)}{S_{T-m-1}\left(\xi_{T-m-1}^{u}-\xi_{T-m-1}^{d}\right)}+k^{m} \sum_{j=a^{d}}^{m} \sum_{I\left(m, j, N_{c_{1}}, \ldots, N_{c_{k}}\right)} M_{2 k}(m, \bar{p}) .
\end{gathered}
$$

Since for any $\omega \in \Omega \quad \xi_{T-m-1}^{d}(\omega)<\xi_{T-m-1}^{u}(\omega)$ then it is evident that $a^{u}<a^{d}$ almost everywhere. If to recall the definitions (2.2.5) and (2.2.6) then

$$
a^{u}=\inf \left\{j:|J|=j, X_{T-m-1} S_{T-m-1} c_{1}^{N_{c_{1}}} \ldots c_{k}^{N_{c_{k}}} u^{j+1} d^{m-j}>K\right\},
$$

where $\mathbf{P}\left\{X_{T-m-1}=c_{1}\right\}=\ldots=\mathbf{P}\left\{X_{T-m-1}=c_{k}\right\}=\frac{1}{k}$, then $a^{d}=a^{u}+1$ almost everywhere. It follows from the fact that

$$
a^{d}=\inf \left\{j:|J|=j, X_{T-m-1} S_{T-m-1} c_{1}^{N_{c_{1}}} \ldots c_{k}^{N_{c_{k}}} u^{j} d^{m-j+1}>K\right\},
$$

and $a^{u}$ is a minimal number from the set $\Gamma_{m}$ for which the inequality

$$
X_{T-m-1} S_{T-m-1} c_{1}^{N_{c_{1}}} \ldots c_{k}^{N_{c_{k}}} u^{a^{u}+1} d^{m-a^{u}}>K
$$

is fulfilled almost sure, so that when we try to find the minimal $a^{d}$, keeping in mind that $a^{d}>a^{u}$, then the following inequality

$$
X_{T-m-1} S_{T-m-1} c_{1}^{N_{c_{1}}} \ldots c_{k}^{N_{c_{k}}} u^{a^{d}} d^{m-a^{d}+1}>K
$$

has to be fulfilled almost sure. The next minimal number after $a^{u}$ is $a^{u}+1$, substituting $a^{d}$ in the latter inequality by $a^{u}+1$ we obtain

$$
X_{T-m-1} S_{T-m-1} c_{1}^{N_{c_{1}}} \ldots c_{k}^{N_{c_{k}}} u^{a^{u}+1} d^{m-a^{u}}>K
$$




\subsection{Multinomial option pricing}

which is already known as a true one. So, $a^{d}=a^{u}+1$. Now we can calculate the number of bonds in portfolio at time $t=T-m-1$. For $c=\left(c_{1}, \ldots, c_{k}\right)$

$$
\begin{aligned}
& \beta_{T-m-1}(c)=\frac{\xi_{T-m-1}^{u} \tilde{C}_{T-m}^{\xi_{T-m-1}^{d}(c)-\xi_{T-m-1}^{d} \tilde{C}_{T-m}^{\xi_{T-m-1}^{u}}(c)}}{\hat{r}\left(\xi_{T-m-1}^{u}-\xi_{T-m-1}^{d}\right)}=\frac{k^{m}}{\hat{r}\left(\xi_{T-m-1}^{u}-\xi_{T-m-1}^{d}\right)} \\
& {\left[\frac{\xi_{T-m-1}^{u}}{\hat{r} m} \sum_{j=a^{d}}^{m} \sum_{I\left(m, j, N_{c_{1}}, \ldots, N_{c_{k}}\right)} M_{2 k}(m, p)\left(\xi_{T-m-1}^{d} S_{T-m-1} \phi_{2 k}(m, c)-K\right)\right.} \\
& -\frac{\xi_{T-m-1}^{d}}{\hat{r}^{m}} \sum_{I\left(m, a^{u}, N_{c_{1}}, \ldots, N_{c_{k}}\right)} M_{2 k}(m, p)\left(\xi_{T-m-1}^{u} S_{T-m-1} \phi_{2 k}(m, c)-K\right) \\
& \left.-\frac{\xi_{T-m-1}^{d}}{\hat{r}^{m}} \sum_{j=a^{d}} \sum_{I\left(m, j, N_{c_{1}}, \ldots, N_{c_{k}}\right)} M_{2 k}(m, p)\left(\xi_{T-m-1}^{u} S_{T-m-1} \phi_{2 k}(m, c)-K\right)\right] \\
& =-\frac{K k^{m}}{\hat{r} m+1} \sum_{j=a^{d}} \sum_{I\left(m, j, N_{c_{1}}, \ldots, N_{c_{k}}\right)} M_{2 k}(m, p)-\frac{k^{m} \delta \xi_{T-m-1}^{d} \Delta_{m}\left(\xi_{T-m-1}^{u} S_{T-m-1}, a^{u}, c\right)}{\hat{r}\left(\xi_{T-m-1}^{u}-\xi_{T-m-1}^{d}\right)} .
\end{aligned}
$$

Without loss of generality we assume that $c_{i_{T-m-1}}=c_{1}$, then for any $c=\left(c_{1}, \ldots, c_{k}\right) \in \mathbb{R}^{k}$

$$
\tilde{C}_{T-m-1}(c)=E\left\{C_{T-m-1} \mid\left(X_{i}\right)_{i=T-m-1}^{T}=\left(c_{j_{i}}\right)_{i=T-m-1}^{T}, c_{j_{i}} \in\left\{c_{1}, \ldots, c_{k}\right\}\right\} .
$$

Here, the condition $\left\{\left(X_{i}\right)_{i=T-m-1}^{T}=\left(c_{j_{i}}\right)_{i=T-m-1}^{T}\right\}$ implies that numbers $N_{c_{2}}, \ldots, N_{c_{k}}$ are the same which were involved in $\tilde{C}_{T-m}$, but $N_{c_{1}}$ has been increased by one.

Let the set $D_{j, m^{+}}\left(x, c_{1}, \ldots, c_{k}\right)$ denotes the set with the same properties as $D_{j, m}\left(x, c_{1}, \ldots, x_{k}\right)$ defined by $(2.2 .4)$ but with additional condition that the element $m \in J_{1}$ and the set $D_{j, m^{-}}\left(x, c_{1}, \ldots, c_{k}\right)$ is also the set with all properties of set $D_{j, m}\left(x, c_{1}, \ldots, c_{k}\right)$, but with additional condition that the element $m \in J_{k+1}$. Thus, the sets $D_{j, m^{+}}\left(x, c_{1}, \ldots, c_{k}\right)$ and $D_{j, m^{-}}\left(x, c_{1}, \ldots, c_{k}\right)$ are mutually exclusive. 


\subsection{Multinomial option pricing}

Now we can calculate

$$
\begin{aligned}
& \tilde{C}_{T-m-1}(c)=\tilde{C}_{T-m}^{\xi_{T-m-1}^{u}} p_{T-m-1}+\tilde{C}_{T-m}^{\xi_{T-m-1}^{d}} q_{T-m-1} \\
& =\frac{k^{m+1}}{\hat{r}^{m+1}} \sum_{j=a^{u}}^{m} \sum_{I\left(m, j, N_{\left.c_{1}+1, N_{c_{2}}, \ldots, N_{c_{k}}\right)}\right.} \frac{m !}{m_{1} ! \ldots m_{2 k} !} p_{1} P_{1}^{2 k}(m)\left(S_{T-m-1} c_{1} u \phi_{2 k}(m, c)-K\right) \\
& +\frac{k^{m+1}}{\hat{r}^{m+1}} \sum_{j=a^{d}}^{m} \sum_{I\left(m, j, N_{c_{1}}+1, N_{c_{2}}, \ldots, N_{c_{k}}\right)} \frac{m !}{m_{1} ! \ldots m_{2 k} !} P_{1}^{2 k}(m) p_{k+1}\left(S_{T-m-1} c_{1} d \phi_{2 k}(m, c)-K\right) \\
& =\frac{k^{m+1}}{\hat{r}^{m+1}} \sum_{j=0}^{m} \sum_{D_{j, m}\left(S_{T-m-1} c_{1} u, c_{1}, \ldots, c_{k}\right)} p_{1} P_{1}^{2 k}(m)\left(S_{T-m-1} c_{1} u \phi_{2 k}(m, c)-K\right)^{+} \\
& +\frac{k^{m+1}}{\hat{r}^{m+1}} \sum_{j=0}^{m} \sum_{D_{j, m}\left(S_{T-m-1} c_{1} d, c_{1}, \ldots, c_{k}\right)} P_{1}^{2 k}(m) p_{k+1}\left(S_{T-m-1} c_{1} d \phi_{2 k}(m, c)-K\right)^{+} .
\end{aligned}
$$

It follows that

$$
\begin{aligned}
& \tilde{C}_{T-m-1}(c) \\
& =\frac{k^{m+1}}{\hat{r}^{m+1}} \sum_{j=0}^{m+1} \sum_{D_{j,(m+1)+}} \sum_{\left(S_{T-m-1}, c_{1}, \ldots, c_{k}\right)} P_{1}^{2 k}(m+1)\left(S_{T-m-1} \phi_{2 k}(m+1, c)-K\right)^{+} \\
& +\frac{k^{m+1}}{\hat{r}^{m+1}} \sum_{j=0}^{m+1} \sum_{D_{j,(m+1)}-\left(S_{T-m-1}, c_{1}, \ldots, c_{k}\right)} P_{1}^{2 k}(m+1)\left(S_{T-m-1} \phi_{2 k}(m+1, c)-K\right)^{+} \\
& =\frac{k^{m+1}}{\hat{r}^{m+1}} \sum_{j=0}^{m+1} \sum_{D_{j,(m+1)}\left(S_{T-m-1}, c_{1}, \ldots, c_{k}\right)} P_{1}^{2 k}(m+1)\left(S_{T-m-1} \phi_{2 k}(m+1, c)-K\right)^{+} \\
& =\frac{k^{m+1}}{\hat{r}^{m+1}} \sum_{j=0}^{m+1} \sum_{I\left(m+1, j, N_{c_{1}}+1, N_{c_{2}}, \ldots, N_{c_{k}}\right)} M_{2 k}(m+1, p)\left(S_{T-m-1} \phi_{2 k}(m+1, p)-K\right)^{+} .
\end{aligned}
$$

The proposition is proved.

We give some examples in order to make clear the meaning of Proposition 2.3.1 and show that (2.3.1) is the generalization of classical Cox-Ross-Rubinstein [20] option pricing formula. 


\subsection{Multinomial option pricing}

Example 2.3.2. If the condition

$$
\mathbf{P}\left\{X_{i} \in \mathbb{C}_{1}\right\}=1, \quad i=T-m, \ldots, T
$$

is satisfied, then

$$
\tilde{C}_{T-m}\left(c_{1}\right)=S_{T-m} \sum_{j=a}^{m} \sum_{I(m, j)} \frac{m !}{m_{1} ! m_{2} !} \bar{p}_{1}^{m_{1}} \bar{p}_{2}^{m_{2}}-\frac{K}{\hat{r}^{m}} \sum_{j=a}^{m} \sum_{I(m, j)} \frac{m !}{m_{1} ! m_{2} !} p_{1}^{m_{1}} p_{2}^{m_{2}}
$$

where $a=a\left(S_{T-m-1}\right)$,

$$
a(x):=\inf \left\{j: I_{j, m}\left(x, N_{c_{1}}\right) \neq \emptyset\right\}
$$

where

$$
\begin{aligned}
I_{j, m}\left(x, N_{c_{1}}\right): & =\left\{J \in \Gamma_{m}:|J|=j, x c_{1}^{N_{c_{1}}} u^{j} d^{m-j}>K\right\}, \\
p_{1}\left(c_{1}\right) & =\frac{\hat{r}-c_{1} d}{c_{1}(u-d)}, \quad p_{2}\left(c_{2}\right)=\frac{c_{1} u-\hat{r}}{c_{1}(u-d)}, \\
\bar{p}_{1}\left(c_{1}\right) & =\frac{c_{1} u}{\hat{r}} p_{1}\left(c_{1}\right), \quad \bar{p}_{2}\left(c_{2}\right)=\frac{c_{1} d}{\hat{r}} p_{2}\left(c_{2}\right)
\end{aligned}
$$

and $p_{1}+p_{2}=1, \bar{p}_{1}+\bar{p}_{2}=1$;

$$
I(m, j)=I\left(m, j, N_{c_{1}}\right):=\left\{\begin{array}{l}
\left(m_{1}, m_{2}\right): m_{i} \in \Gamma_{m}, i=1,2 ; \\
m_{1}+m_{2}=m: m_{1}=j, N_{c_{1}}=m_{1}+m_{2}=m
\end{array}\right\} .
$$

At time $t=T-m-1$ the unique replicating strategy $\phi$ is given by

$$
\begin{aligned}
& \alpha_{T-m-1}\left(c_{1}\right)=\sum_{j=a^{d}} \sum_{I(m, j)} \frac{m !}{m_{1} ! m_{2} !} \bar{p}_{1}^{m_{1}} \bar{p}_{2}^{m_{2}}+\frac{\delta \Delta_{m}\left(\xi_{T-m-1}^{u} S_{T-m-1}, a^{u}, c_{1}\right)}{S_{T-m-1}\left(\xi_{T-m-1}^{u}-\xi_{T-m-1}^{d}\right)}, \\
& \beta_{T-m-1}\left(c_{1}\right)=-\frac{K}{\hat{r}^{m+1}} \sum_{j=a^{d}} \sum_{I(m, j)} \frac{m !}{m_{1} ! m_{2} !} p_{1}^{m_{1}} p_{2}^{m_{2}}-\frac{\delta \Delta_{m}\left(\xi_{T-m-1}^{u} S_{T-m-1}, a^{u}, c_{1}\right)}{\hat{r}\left(\xi_{T-m-1}^{u}-\xi_{T-m-1}^{d}\right)},
\end{aligned}
$$

where

$$
\Delta_{m}\left(x, j, c_{1}\right):=\frac{1}{\hat{r}^{m}} \sum_{I(m, j)} \frac{m !}{m_{1} ! m_{2} !} \bar{p}_{1}^{m_{1}} \bar{p}_{2}^{m_{2}}\left(x\left(c_{1} u\right)^{m_{1}}\left(c_{1} d\right)^{m_{2}}-K\right),
$$

$a^{u}=a\left(\xi_{T-m-1}^{u} S_{T-m-1}\right), \quad a^{d}=a\left(\xi_{T-m-1}^{d} S_{T-m-1}\right)$ and $\delta=0$ if $a^{u}=a^{d}$ (otherwise $\delta=1)$. 
Remark 2.3.3. When $c_{1}=1$ with probability 1 then we have the classical Cox-Ross-Rubinstein formula for option price which is given by (2.3.4). Because of conditions on $m_{1}$ and $m_{2}$ the coefficient $\frac{m !}{m_{1} ! m_{2} !}$ is a binomial coefficient. It is important to notice that multinomial CRR (2.3.11) does not depend on the subjective probability $p$. Intuitively it means that the pricing formula does not depend on the investor's attitudes toward risk. The only assumption about investors is that they all prefer more wealth to less. The assumptions about absence of arbitrage provide so called partial equilibrium approach, which is frequently used in economical literature in relation to arbitrage pricing.

It was noticed (see e.g. [48]) that formula (2.3.4) in Example 2.3.2 can be written as follows

$$
C_{T-m}=S_{T-m} \overline{\mathbb{P}}\left\{S_{m}>K\right\}-K \hat{r}^{-(T-m)} \mathbb{P}^{*}\left\{S_{m}>K\right\}
$$

where $\overline{\mathbb{P}}$ and $\mathbb{P}^{*}$ are martingale measures corresponding to the choice of stock price and bond price, respectively. The representation (2.3.7) can also be used to derive Black-Scholes formula when stock price follows Geometric Brownian Motion. In this case one has to use the general theory of limit distributions of independent identically distributed random variables.

Remark 2.3.4 For the case when $k=1$ we have the representation (2.3.7). So, there are at least two possibilities to obtain the continuous-time BlackScholes option price formula: from discrete-time CRR model when the number of trading periods increases, i.e. $m \rightarrow \infty$, and from (2.3.7). One can see that the $\log$ of stock price is represented as a sum of independent identically binomially distributed random variables, so that it is possible to derive all possible asymptotical distributions for $\overline{\mathbb{P}}\left\{S_{m}>K\right\}$ and $\mathbb{P}^{*}\left\{S_{m}>K\right\}$, as $m \rightarrow \infty$.

In the case when $k \geq 2$ there is no such representation which is analogous to (2.3.7). So, one can not use the general theory of limit theorems and will need corresponding asymptotic procedure to obtain continuous approximation of multinomial CRR option price. 


\subsection{Multinomial option pricing}

We also consider here as an example the case when the condition

$$
\mathbf{P}\left\{X_{i} \in\left\{c_{1}, c_{2}\right\}\right\}=1, \quad i=T-m, \ldots, T
$$

is satisfied, i.e. the number of possible values of $\left(X_{t}\right)_{t \leq T}$ is $k=2$.

Example 2.3.4. If the sequence $X_{T-m}, \ldots, X_{T}$ satisfies the condition

$$
\mathbf{P}\left\{X_{i} \in \mathbb{C}_{2}\right\}=1, \quad i=T-m, \ldots, T
$$

then

$$
\tilde{C}_{T-m}\left(c_{1}, c_{2}\right)=2^{m}\left(S_{T-m} \sum_{j=a}^{m} \sum_{I\left(m, j, N_{c_{1}}, N_{c_{2}}\right)} M_{4}(m, \bar{p})-\frac{K}{\hat{r}^{m}} \sum_{j=a}^{m} \sum_{I\left(m, j, N_{c_{1}}, N_{c_{2}}\right)} M_{4}(m, p)\right),
$$

where $a=a\left(S_{T-m}\right)$,

$$
a(x):=\inf \left\{j: I_{j, m}\left(x, N_{c_{1}}, N_{c_{2}}\right) \neq \emptyset\right\}
$$

where

$$
\begin{gathered}
I_{j, m}\left(x, N_{c_{1}}, N_{c_{2}}\right):=\left\{J \in \Gamma_{m}: \quad|J|=j, x c_{1}^{N_{c_{1}}} c_{2}^{N_{c_{2}}} u^{j} d^{m-j}>K\right\} \\
p_{1}=\frac{1}{2} \frac{\hat{r}-c_{1} d}{c_{1}(u-d)}, \quad p_{2}=\frac{1}{2} \frac{\hat{r}-c_{2} d}{c_{2}(u-d)}, \quad p_{3}=\frac{1}{2} \frac{c_{1} u-\hat{r}}{c_{1}(u-d)}, \quad p_{4}=\frac{1}{2} \frac{c_{2} u-\hat{r}}{c_{2}(u-d)}, \\
\bar{p}_{1}=\frac{c_{1} u}{\hat{r}} p_{1}, \quad \bar{p}_{2}=\frac{c_{2} u}{\hat{r}} p_{2}, \quad \bar{p}_{3}=\frac{c_{1} d}{\hat{r}} p_{1}, \quad \bar{p}_{4}=\frac{c_{2} d}{\hat{r}} p_{4}
\end{gathered}
$$

and

$$
\begin{gathered}
p_{1}+p_{3}=p_{2}+p_{4}=\frac{1}{2}, \quad \bar{p}_{1}+\bar{p}_{3}=\bar{p}_{2}+\bar{p}_{4}=\frac{1}{2} \\
I\left(m, j, N_{c_{1}}, N_{c_{2}}\right):=\left\{\begin{array}{l}
\left(m_{1}, m_{2}, m_{3}, m_{4}\right): m_{i} \in \Gamma_{m}, i=1, \ldots, 4 ; \\
m_{1}+m_{2}+m_{3}+m_{4}=m: m_{1}+m_{2}=j \\
N_{c_{1}}=m_{1}+m_{3}, N_{c_{2}}=m_{2}+m_{4}
\end{array}\right\} .
\end{gathered}
$$

At time $t=T-m-1$ the unique replicating strategy $\phi$ is given by

$$
\alpha_{T-m-1}\left(c_{1}, c_{2}\right)=2^{m} \sum_{j=a^{d}} \sum_{I\left(m, j, N_{c_{1}}, N_{c_{2}}\right)} M_{4}(m, \bar{p})+\frac{2^{m} \delta \Delta_{m}\left(\xi_{T-m-1}^{u} S_{T-m-1}, a^{u}, c_{1}, c_{2}\right)}{S_{T-m-1}\left(\xi_{T-m-1}^{u}-\xi_{T-m-1}^{d}\right)},
$$




$$
\beta_{T-m-1}\left(c_{1}, c_{2}\right)=-\frac{2^{m} K}{\hat{r}^{m+1}} \sum_{j=a^{d}} \sum_{I\left(m, j, N_{c_{1}}, N_{c_{2}}\right)} M_{4}(m, p)-\frac{2^{m} \delta \Delta_{m}\left(\xi_{T-m-1}^{u} S_{T-m-1}, a^{u}, c_{1}, c_{2}\right)}{\hat{r}\left(\xi_{T-m-1}^{u}-\xi_{T-m-1}^{d}\right)}
$$

where

$$
\Delta_{m}\left(x, j, c_{1}, c_{2}\right):=\frac{2^{m}}{\hat{r}^{m}} \sum_{I\left(m, j, N_{c_{1}}, N_{c_{2}}\right)} M_{4}(m, \bar{p})\left(x \phi_{4}(m, c)-K\right),
$$

$a^{u}=a\left(\xi_{T-m-1}^{u} S_{T-m-1}\right), \quad a^{d}=a\left(\xi_{T-m-1}^{d} S_{T-m-1}\right)$ and $\delta=0$ if $a^{u}=a^{d}$ (otherwise $\delta=1)$.

Remark 2.3.5 Already in case when $k=2$, the option price formula is quite complicated and it is not clear how one could obtain this form of option price using pure probabilistic approach.

Numerical examples and multinomiality parameters estimation procedure will be described in Chapter 4, examples will have been obtained in the case $k=2$. 


\section{Chapter 3}

\section{Convergence of Generalized Multinomial Option Price Model}

Construction of a corresponding asymptotic procedure allowed Cox et al. [20] to obtain the classical Black-Scholes formula [7] from their discrete-time option pricing model. In this chapter we apply the similar approach to generalized discrete-time multinomial option pricing model (2.3.1) and consider the limit case of multinomial CRR option price. Discrete-time stock price process used in classical CRR model is the approximation of Geometrical Brownian Motion which is under certain assumptions the case in our model too. We assume that random sequence $\left(X_{t}\right)_{t \leq T}$ embedded in the generalized stock price model (1.1.2)

$$
S_{t}=S_{t-1} X_{t-1} \nu_{t-1}, \quad \forall \quad t \leq T
$$

take values in the multinomilality parameters set, $\mathbb{C}_{k}$ defined by $(2.2 .1)$ and find conditions, under which, there is a convergence of the multinomial option price to its continuous analog. The construction of asymptotic procedure and specification of the features of multinomiality parameters set will allow us to obtain the generalized multinomial Black-Scholes option price formula with respect to the generalization of the stock price model (1.1.2) when one can observe different changes factors of jump sizes at each time moment.

It is necessary to point out that in classical binomial stock price model used in classical CRR option price model the stock price process was modelled by the sequence of random variables taking only two values $u$ and $d$. In our model the stock price process is modelled by the sequence of random variables which 


\subsection{Averaging over all possible multinomiality parameters sets}

can take more values than those two. So that the generalized Black-Scholes formula depends on the multinomiality parameters which gives more flexibility in the applications. The classical Black-Scholes option price can be obtained as a consequence from the generalized multinomial option price formula accurate to the constant when there is only one multinomiality parameter.

\subsection{Averaging over all possible multinomiality pa- rameters sets}

The multinomial option price $\tilde{C}_{t},, t \leq T$ given by (2.3.1) was obtained due to the conditioning on the values of random sequence $X_{T-m}, \ldots, X_{T}$, where each element of this sequence $X_{t}, t=T-m, \ldots, T$ takes values in the multinomiality parameters set $\mathbb{C}_{k}, k \leq m$ defined by (2.2.1). Thus, the option price is dependent on the certain combination of values of random sequence $N_{c_{1}}, \ldots, N_{c_{k}}$ (the Definition 2.2.1).

In practice it is not known exactly, which combination of $N_{c_{1}}, \ldots, N_{c_{k}}$ occurs and hence, one should consider the averaging over all possible combinations of $N_{c_{1}}, \ldots, N_{c_{k}}$ or, which is equivalent, the averaging over all possible sequences of multinomiality parameters $\left(c_{j_{i}}\right)_{i=T-m}^{T}, c_{j_{i}} \in \mathbb{C}_{k}$.

For any $c=\left(c_{1}, \ldots, c_{k}\right)$ we consider the expectation

$$
\hat{C}_{T-m}(c):=E_{N_{c_{1}}, \ldots, N_{c_{k}}}\left\{\tilde{C}_{T-m}\left(c, N_{c_{1}}, \ldots, N_{c_{k}}\right)\right\}=E\left\{E\left\{C_{T-m} \mid\left(X_{i}\right)_{i=T-m}^{T} \in \mathbb{C}_{k}\right\}\right\} .
$$

Define the set of indexes sets which does not depend on $N_{c_{1}}, \ldots, N_{c_{k}}$ anymore.

$$
I(m, j):=\left\{\begin{array}{l}
\left(m_{1}, \ldots, m_{2 k}\right): m_{i} \in \Gamma_{m}, i=1, \ldots, 2 k ; \\
m_{1}+\ldots+m_{2 k}=m: m_{1}+\ldots+m_{k}=j
\end{array}\right\},
$$

so that (3.1.1) can be written as

$$
\hat{C}_{T-m}(c)=S_{T-m} \sum_{j=a}^{m} \sum_{I(m, j)} M_{2 k}(m, \bar{p})-\frac{K}{\hat{r}^{m}} \sum_{j=a}^{m} \sum_{I(m, j)} M_{2 k}(m, p),
$$

where $M_{2 k}(m, p)$ is defined by (2.2.8) and $a=a\left(S_{T-m}\right)$ is defined by (2.2.6). 


\section{$3.2 \quad$ Asymptotic procedure}

Choosing the proper sequence of (3.1.3) it is possible to obtain the corresponding limit which one refers as to generalized Black-Scholes option valuation formula.

\section{$3.2 \quad$ Asymptotic procedure}

Consider $T>0$ is some arbitrary real number. Take any $n$ of the form $n=2^{s}, s$ is a natural number. Then divide the interval $[0, T]$ into $n$ equal subintervals $I_{j}$ of length $\Delta_{n}=\frac{T}{n}$, namely $I_{j}=\left[j \Delta_{n},(j+1) \Delta_{n}\right]$ for $j=0, \ldots, n-1$.

Our purpose is to calculate the asymptotic value of the European call option price when the number of time periods, or number of time intervals $I_{j}, j=0, \ldots, n-1$ increases, as $n \rightarrow \infty$. Assume that

$$
t=j \Delta_{n}=\frac{j T}{n}=\frac{j T}{2^{s}}
$$

for some natural $s$ and $j$, this means that $t$ is an arbitrary dyadic number from the interval $[0, T]$.

Introduce the sequence $m_{n}(t)$ by setting

$$
m_{n}(t)=\frac{T-t}{T} n, \quad \forall n \in \mathbb{N},
$$

where $\mathbb{N}$ denotes the set of natural numbers. Apparently, the sequence $m_{n}(t)$ has natural values for $n$ sufficiently large. Further, for the convenience of notations we will denote the sequence $m_{n}(t)$ as $m_{n}$ or just $m$.

Moreover, $T-t=m_{n} \Delta_{n}$, so that $m_{n}$ represents the number of trading periods in the interval $[t, T]$ and $m \rightarrow \infty$, as $n \rightarrow \infty$.

We examine the asymptotic properties of $\hat{C}_{T-m}(c)$ defined by (3.1.3) when the number of steps goes to infinity and, simultaneously, the size of time steps tends to zero. We should notice that the time index in $\hat{C}_{T-m}, T-m$ is natural number. In order to construct the sequence of $\hat{C}_{T-m}$ for any $T>0$ and $m \in[0, T]$ we consider $T-m=n \Delta_{n}-m \Delta_{n}=t, t \in[0, T]$ and $t=j \Delta_{n}$ for any natural $j$.

Let $r_{n}$ be the riskless rate of return over each interval $I_{j}$, then the riskfree asset price process is given by

$$
B_{j \Delta_{n}}=\left(1+r_{n}\right)^{j}, \quad j=0, \ldots, n
$$




\section{$3.2 \quad$ Asymptotic procedure}

The stock price (risky asset) process is given by

$$
S_{(j+1) \Delta_{n}}=\xi_{j, n} S_{j \Delta_{n}}, \quad j=0, \ldots, n-1
$$

where

$$
\xi_{j, n}=X_{j, n} \exp \left\{u_{n} \eta_{j}+d_{n}\left(1-\eta_{j}\right)\right\}, \quad j=0, \ldots, n-1,
$$

where random variables $\eta_{j}, j=0, \ldots, n-1$ are independent identically Bernoulli distributed random variables, i.e. $\eta_{j}$ take values in the set $\{0,1\}$ with corresponding probabilities $p$ and $1-p$. We assume that random variables $X_{j, n}$ and $\eta_{j}, j=0, \ldots, n-1$ are mutually independent.

$$
\begin{gathered}
\mathbf{P}\left\{\xi_{j, n}=u_{i, n}\right\}=\mathbf{P}\left\{\xi_{j, n}=x_{i, n} \exp \left\{u_{n}\right\} \mid X_{j, n}=x_{i, n}\right\} \\
=1-\mathbf{P}\left\{\xi_{j, n}=x_{i, n} \exp \left\{d_{n}\right\} \mid X_{j, n}=x_{i, n}\right\}=1-\mathbf{P}\left\{\xi_{j, n}=d_{i, n}\right\}=p, \\
j=0, \ldots, n-1, i=1, \ldots, k .
\end{gathered}
$$

Also, impose specific restrictions on the asymptotic behavior of quantities $r_{n}$, $x_{n} e^{u_{n}}$ and $x_{n} e^{d_{n}}$. Assume that we have a sequence of multinomiality parameters sets $\mathbb{C}_{k, n}=\left\{c_{1, n}, \ldots, c_{k, n}\right\}$. So that, $x_{n} \in \mathbb{C}_{k, n}$.

Hence, we have that

$$
u_{i, n}=c_{i, n} e^{u_{n}} \text { and } d_{i, n}=c_{i, n} e^{d_{n}}, i=1, \ldots, k .
$$

Also, the corresponding sequences are represented as

$$
\begin{gathered}
\hat{r}_{n}=1+r_{n}=e^{r \Delta_{n}} \\
u_{i, n}=\left(c_{i} e\right)^{\sigma \sqrt{\Delta_{n}}}=e^{\left(\ln c_{i}+1\right) \sigma \sqrt{\Delta_{n}}} \\
d_{i, n}=\left(c_{i} e\right)^{-\sigma \sqrt{\Delta_{n}}}=e^{-\left(\ln c_{i}+1\right) \sigma \sqrt{\Delta_{n}}}, \quad i=1, \ldots, k,
\end{gathered}
$$

where $r>0$ and $\sigma>0$ are real numbers.

The values $p_{j, n}, j=1, \ldots, 2 k$ are expressed via $r_{n}, d_{i, n}$ and $u_{i, n}, i=1, \ldots, k$, which we will denote as $p_{j}$ for the convenience of notations, and form $2 k$ sequences $\left(p_{j, n}\right)=\left(p_{j}\right), j=1, \ldots, 2 k$.

$$
\begin{gathered}
p_{j, n}=\frac{1}{k} \frac{\hat{r}_{n}-d_{j, n}}{u_{j, n}-d_{j, n}}, \quad j=1, \ldots, k, \\
p_{j, n}=\frac{1}{k} \frac{u_{j-k, n}-\hat{r}_{n}}{u_{j-k, n}-d_{j-k, n}}, \quad j=k+1, \ldots, 2 k .
\end{gathered}
$$




\subsection{Multinomial CRR volatility modelling}

The constant $r>0$ is the risk-free interest rate of return yielded by a risk-free investment which is bond and $\sigma>0$ is the standard deviation of the log return of the risky investment which is stock price. In other words, $\sigma$ is called a volatility parameter of the return of stock prices. The classical Black and Scholes formula [7] for European option price is given by

$$
C_{t}=S_{t} \Phi\left(\frac{\ln \frac{S_{t}}{K}+\left(r+\frac{1}{2} \sigma^{2}\right)(T-t)}{\sigma \sqrt{T-t}}\right)-K e^{-(T-t)} \Phi\left(\frac{\ln \frac{S_{t}}{K}+\left(r-\frac{1}{2} \sigma^{2}\right)(T-t)}{\sigma \sqrt{T-t}}\right), \quad t \in[0, T]
$$

where $\Phi(x)=\frac{1}{\sqrt{2 \pi}} \int_{-\infty}^{x} e^{-\frac{u^{2}}{2}} d u, \forall x \in \mathbb{R}, T$ is expiry date and $K$ is strike price.

In order to calculate the option price using discrete-time CRR option price [20] one should know the quantities $T, m, S_{T-m}, r, \sigma$, when the classical Black-Scholes option price formula (3.3.1) [7] is used then one should know $T, t, S_{t}, r, \sigma$. All quantities are observable directly except for the volatility parameter $\sigma$.

The constant volatility assumed in Black-Scholes model was not satisfactory anymore. In 1977 Galai [26] confirmed the fact about the deviations in the Black-Scholes model. Also, it was noticed (see [8]) that due to the model, the options written on stocks with high (low, respectively) historical volatilities are overpriced (underpriced, respectively). It was suggested that the effectiveness of the model depends how good the volatility can be predicted. So, the relevant problem is to estimate volatility parameter of the return of stock price.

Historical volatility is estimated quite straightforward and there are some important points necessary to mention. Usually, to reduce the estimation bias arising from the sampling error, it is natural to increase the sample size, either using longer series of historical observations or increasing the frequency of observations. But there is some evidence that the variance in financial data is non-stationary and it might be that increase in sample size can make estimate even worse. Some studies devoted to historical volatility one can find in works [15], [51], [42], [69], [17].

The implied volatility is obtained from the observed at the market option price. It is possible, because the Black-Scholes price of European call option is an increasing function of the volatility ( [25] (pp.255)) and all other parameters 
defining the option price are usually known. This implied volatility $\sigma_{t}, t \in[0, T]$ is derived from (3.3.1) and depends on strike price $K$, time to maturity $T-t$. An average of these implied volatilities is used as a measure of market forecasts of return volatility. Beckers in 1981 [6] found that the implied volatility was a better least-squared predictor of the actual volatility than the historical volatility. Some aspects of implied volatility are discussed in [33].

In continuous-time models it was suggested that the volatility should be modeled as a stochastic process. Such models are called the stochastic volatility models and the volatility process is described by stochastic differential equation, see e.g. [61], [62], [16].

The discrete-time approach to model stochastic volatility is based on autoregressive random variance models (ARV models) (see e.g. [72], [43]) and on so-called ARCH (or GARCH) models (see e.g. [23], [49], [68]). Much more of literature review about volatility studies one can find in [48] or [60].

The above survey about volatility parameter studies shows the importance of that for financial markets. One should notice that originally there were no assumptions about volatility parameter in multinomial CRR model of stock price. After the asymptotic procedure was introduced it appeared that the volatility parameter of stock return is modelled as stochastic process.

Theorem 3.3.1 If it is possible to construct the asymptotic procedure in that way that there is a sequence of multinomial CRR option prices $\hat{C}_{t, n}(c), t=0, \ldots, T$ from (3.1.1) and $\forall i=1, \ldots, k, u_{i, n}=\frac{1}{d_{i, n}}$, then the volatility process is given by

$$
\sigma_{t}=\sigma\left(\ln X_{t}+1\right), \forall t=0, \ldots, T
$$

where $X_{t}, t=0, \ldots, T$ take values in the multinomiality parameters set $\mathbb{C}_{k}$, defined in (2.2.1).

Proof. Due to definitions of $u_{i, n}, d_{i, n}, i=1, \ldots, k$ in (3.2.5) and (3.2.6) via the volatility parameter $\sigma$ and multinomiality parameters $c_{1}, \ldots, c_{k}$ the statement of the theorem is the true one. 


\subsection{Limit theorems for multinomial distribution. Auxiliary results}

In this section we will use the following notations

$$
p\left(m_{1}, \ldots, m_{k}\right):=\frac{m !}{m_{1} ! \ldots m_{k} !} p_{1}^{m_{1}} \ldots p_{k}^{m_{k}}, \forall k \geq 2
$$

so that

$$
p\left(m_{1}+m_{2}, m_{3}, \ldots, m_{k}\right)=\frac{m !}{\left(m_{1}+m_{2}\right) ! m_{3} ! \ldots m_{k} !}\left(p_{1}+p_{2}\right)^{m_{1}+m_{2}} p_{3}^{m_{3}} \ldots p_{k}^{m_{k}},
$$

where $\sum_{i=1}^{k} p_{i}=1, \sum_{i=1}^{k} m_{i}=m$.

Further, we will use the sign " $\asymp "$ to denote the equivalence relation as $m \rightarrow \infty$ in the meaning that

$$
a(m) \asymp b(m) \text { if and only if } \frac{a(m)}{b(m)} \longrightarrow 1, \quad \text { as } \quad m \rightarrow \infty .
$$

When $m \nrightarrow \infty$, then it is assumed that $a(m)=b(m)$.

Lemma 3.4.1. If $p_{1} \asymp p_{2} \asymp \ldots \asymp p_{k}, k \geq 2$, then

$$
\sum_{m_{1}, m_{2}, \ldots, m_{k-1}} p\left(m_{1}, \ldots, m_{k}\right) \asymp \sum_{m_{1}+m_{2}, m_{3}, \ldots, m_{k-1}} p\left(m_{1}+m_{2}, m_{3}, \ldots, m_{k}\right) .
$$

Proof. We apply the method of mathematical induction with respect to $k$. The base of induction is the case when $k=2$, then

$$
\sum_{m_{1}=0}^{n} \frac{m !}{m_{1} ! m_{2} !} p_{1}^{m_{1}} p_{2}^{m_{2}}=1 \quad \text { and } \quad \frac{m !}{\left(m_{1}+m_{2}\right) !}\left(p_{1}+p_{2}\right)^{m_{1}+m_{2}}=1 .
$$

The transition of induction is when we assume that the statement is true for $m_{1}, \ldots, m_{k-1}$ and the question is if the statement is true for $m_{1}, \ldots, m_{k-1}, m_{k}$.

$$
\sum_{m_{1}, m_{2}, \ldots, m_{k}} \frac{m !}{m_{1} ! \ldots m_{k} !} p_{1}^{m_{1}} \ldots p_{k}^{m_{k}}=\sum_{m_{1}, m_{2}, \ldots, m_{k-1}} \frac{m !}{m_{1} ! \ldots m_{k-1} !} p_{1}^{m_{1}} \ldots p_{k-1}^{m_{k-1}} \sum_{m_{k}} \frac{1}{m_{k} !} p_{k}^{m_{k}}
$$




\subsection{Limit theorems for multinomial distribution. Auxiliary results}

$$
\begin{aligned}
& =\sum_{m_{1}+m_{2}, m_{3}, \ldots, m_{k-1}} \frac{m !}{\left(m_{1}+m_{2}\right) ! m_{3} ! \ldots m_{k-1} !}\left(p_{1}+p_{2}\right)^{m_{1}+m_{2}} p_{3}^{m_{3}} \ldots p_{k-1}^{m_{k-1}} \sum_{m_{k}} \frac{1}{m_{k} !} p_{k}^{m_{k}} \\
& =\sum_{m_{1}+m_{2}, m_{3}, \ldots, m_{k}} \frac{m !}{\left(m_{1}+m_{2}\right) ! m_{3} ! \ldots m_{k} !}\left(p_{1}+p_{2}\right)^{m_{1}+m_{2}} p_{3}^{m_{3}} \ldots p_{k}^{m_{k}} .
\end{aligned}
$$

The lemma is proved.

Returning to our model, for $m=m_{1}+\ldots+m_{2 k}, p=\left(p_{1}, \ldots, p_{2 k}\right)$ and any non-negative integer number $x \in \mathbb{Z}^{+}$we define the set

$$
\begin{aligned}
\mathfrak{M}_{2 k}(m, p, x):= & \left\{\begin{array}{l}
\left(m_{1}+\ldots+m_{k}, m_{k+1}, \ldots, m_{2 k}\right): m_{1}+\ldots+m_{2 k}=m \\
x \leq m_{1}+\ldots+m_{k} \leq m\left(p_{1}+\ldots+p_{k}\right)+A m^{\gamma}, \\
m p_{i}+A m^{\gamma} \leq m_{i} \leq m p_{i}+A m^{\gamma}, \forall i=k+1, \ldots, 2 k
\end{array}\right\} \\
= & \left\{\begin{array}{l}
\left(\mathfrak{m}_{1}^{k}, \mathfrak{m}_{k+1}, \ldots, \mathfrak{m}_{2 k}\right): \mathfrak{m}_{1}^{k}+\ldots+\mathfrak{m}_{2 k}=m \\
x \leq \mathfrak{m}_{1}^{k} \leq m\left(p_{1}+\ldots+p_{k}\right)+A m^{\gamma}, \\
m p_{i}+A m^{\gamma} \leq \mathfrak{m}_{i} \leq m p_{i}+A m^{\gamma}, \forall i=k+1, \ldots, 2 k
\end{array}\right\}
\end{aligned}
$$

where $A>0$ is a constant, $\frac{1}{2}<\gamma<\frac{2}{3}, \quad k \leq \frac{m}{2}$.

Introduce the following notations

$$
x_{i}:=\frac{m_{i}-m p_{i}}{\sqrt{m}} i=1, \ldots, 2 k
$$

and

$$
\bar{x}_{i}:=\frac{m_{i}-m \bar{p}_{i}}{\sqrt{m}}, i=1, \ldots, 2 k .
$$

Since $\sum_{i=1}^{2 k} m_{i}=m$ and $\sum_{i=1}^{2 k} p_{i}=\sum_{i=1}^{2 k} \bar{p}_{i}=1$, then $\sum_{i=1}^{2 k} x_{i}=\sum_{i=1}^{2 k} \bar{x}_{i}=0$.

Define the set

$$
\mathbb{X}:=\left\{\left(x_{1}, \ldots, x_{2 k}\right):\left|x_{i}\right| \leq A m^{\frac{1}{2}-\beta} \forall i=1, \ldots, 2 k\right\},
$$

where $A, \beta$ are positive constants and $\frac{1}{3}<\beta<\frac{1}{2}$. 
Proposition 3.4.2. For $x_{i} \in \mathbb{X}, i=1, \ldots, k$, as $n \rightarrow \infty$, the following asymptotic relation holds true

$$
\begin{array}{r}
\hat{C}_{T-m} \asymp \breve{C}_{T-m}=S_{T-m} \sum_{\mathfrak{M}_{2 k}(m, p, x)} \frac{1}{(2 \pi)^{\frac{k}{2}}} \sqrt{\sqrt{\left(\sum_{i=1}^{k} \bar{p}_{i}\right) \bar{p}_{k+1} \ldots \bar{p}_{2 k}}} \exp \left\{\frac{\left(\sum_{i=1}^{k} \bar{x}_{i}\right)^{2}}{\sum_{i=1}^{k} \bar{p}_{i}}+\sum_{i=k+1}^{2 k} \frac{\bar{x}_{i}^{2}}{\bar{p}_{i}}\right\} \\
-\frac{K}{\hat{r}^{m}} \sum_{\mathfrak{M}_{2 k}(m, \bar{p}, x)} \frac{1}{(2 \pi)^{\frac{k}{2}}} \sqrt{\sqrt{\left(\sum_{i=1}^{k} p_{i}\right) p_{k+1} \ldots p_{2 k}}} \exp \left\{\frac{1}{\left.\sum_{i=1}^{k} x_{i}\right)^{2}}+\sum_{i=k+1}^{2 k} \frac{x_{i}^{2}}{p_{i}}\right\} .
\end{array}
$$

Proof. The expression for $\hat{C}_{T-m}(c)$ from (3.1.1) contains multinomial probabilities with parameters $(m, p)$, where $p=\left(p_{1}, \ldots, p_{2 k}\right)$. Due to the limit theorem about multinomial distribution (look [56], p.342, (2.21)) and to Lemma 3.4.1. the statement of the theorem is the true one. We just have to show that the conditions of Lemma 3.4.1 are satisfied.

$$
\begin{gathered}
\lim _{n \rightarrow \infty} p_{1, n}=\lim _{n \rightarrow \infty} \frac{e^{r \Delta_{n}}-e^{-\left(\ln c_{1}+1\right) \sigma \sqrt{\Delta_{n}}}}{k\left(e^{\left(\ln c_{1}+1\right) \sigma \sqrt{\Delta_{n}}}-e^{-\left(\ln c_{1}+1\right) \sigma \sqrt{\Delta_{n}}}\right)} \\
=\lim _{n \rightarrow \infty} \frac{1+r \Delta_{n}+\bar{o}\left(\Delta_{n}\right)-1+\sigma\left(\ln c_{1}+1\right) \sqrt{\Delta_{n}}-\frac{\sigma^{2}\left(\ln c_{1}+1\right)^{2} \Delta_{n}}{2}-\bar{o}\left(\Delta_{n}\right)}{k\left(1+\sigma\left(\ln c_{1}+1\right) \sqrt{\Delta_{n}}+\bar{o}\left(\sqrt{\Delta_{n}}\right)-1+\sigma\left(\ln c_{1}+1\right) \sqrt{\Delta_{n}}-\bar{o}\left(\sqrt{\Delta_{n}}\right)\right)} \\
=\frac{\sigma\left(\ln c_{1}+1\right)^{2} \sqrt{\Delta_{n}}+\left(r-\frac{\sigma^{2}\left(\ln c_{1}+1\right)^{2}}{2}\right) \Delta_{n}}{2 k \sigma\left(\ln c_{1}+1\right) \sqrt{\Delta_{n}}}=\frac{1}{2 k} .
\end{gathered}
$$

Calculation of limits of the others $p_{i, n}$ is similar and shows that $p_{1} \asymp \ldots \asymp p_{2 k} \asymp \frac{1}{2 k}$, as $n \rightarrow \infty$. The proposition is proved.

When $k=1$, then Proposition 3.2.4 is well-known Moivre-Laplace local limit theorem adapted for the option price valuation formula. Our purpose is to calculate the limit of $\hat{C}_{T-m}$ as $n \rightarrow \infty$. 
Denote

$$
\begin{aligned}
& \bar{D}_{2 k-2}(x) \\
& :=(2+k)\left(\sum_{i=1}^{k} \bar{x}_{i}\right)^{2}+2 k\left(\sum_{i=k+1}^{2 k-2} \bar{x}_{i}^{2}\right)+2 k\left(\sum_{i=1}^{k} \bar{x}_{i}\right)\left(\sum_{i=k+1}^{2 k-2} \bar{x}_{i}\right)+k\left(\sum_{i=k+1}^{2 k-2} \bar{x}_{i}\right)^{2} \\
& D_{2 k-2}(x) \\
& :=(2+k)\left(\sum_{i=1}^{k} x_{i}\right)^{2}+2 k\left(\sum_{i=k+1}^{2 k-2} x_{i}^{2}\right)+2 k\left(\sum_{i=1}^{k} x_{i}\right)\left(\sum_{i=k+1}^{2 k-2} x_{i}\right)+k\left(\sum_{i=k+1}^{2 k-2} x_{i}\right)^{2} . \\
& \bar{R}_{2 k-1}(x):=4 k\left(\bar{x}_{2 k-1}+\frac{1}{2}\left(\sum_{i=1}^{2 k-2} \bar{x}_{i}\right)\right)^{2}, \quad R_{2 k-1}(x):=4 k\left(x_{2 k-1}+\frac{1}{2}\left(\sum_{i=1}^{2 k-2} x_{i}\right)\right)^{2} .
\end{aligned}
$$

Further, we will omit $x$ as argument of $\bar{D}_{2 k-2}, D_{2 k-2}, \bar{R}_{2 k-1}$ and $R_{2 k-1}$.

Lemma 3.4.3 Let $p_{1} \asymp \ldots p_{2 k} \asymp \frac{1}{2 k}$, as $n \rightarrow \infty$, then the following relation holds true

$$
\frac{\left(\sum_{i=1}^{k} x_{i}\right)^{2}}{\sum_{i=1}^{k} p_{i}}+\sum_{i=k+1}^{2 k} \frac{x_{i}^{2}}{p_{i}} \asymp D_{2 k-2}+R_{2 k-1}
$$

Proof. The proof of this statement is straightforward calculations. Since $p_{1} \asymp \ldots p_{2 k} \asymp \frac{1}{2 k}$, then

$$
\begin{aligned}
& \frac{\left(x_{1}+\ldots+x_{k}\right)^{2}}{p_{1}+\ldots+p_{k}}+\frac{x_{k+1}^{2}}{p_{k+1}}+\ldots+\frac{x_{2 k}^{2}}{p_{2 k}} \asymp 2\left(\sum_{i=1}^{k} x_{i}\right)^{2}+2 k\left(\sum_{i=k+1}^{2 k-1} x_{i}^{2}\right)+2 k\left(\sum_{i=1}^{2 k-1} x_{i}\right)^{2} \\
& =2\left(\sum_{i=1}^{k} x_{i}\right)^{2}+2 k\left(\sum_{i=k+1}^{2 k-1} x_{i}^{2}\right)+2 k\left(\sum_{i=1}^{2 k-2} x_{i}\right)^{2}+4 k\left(\sum_{i=1}^{2 k-2} x_{i}\right) x_{2 k-1}+2 k x_{2 k-1}^{2} \\
& =2\left(\sum_{i=1}^{k} x_{i}\right)^{2}+2 k x_{2 k-1}^{2}+2 k\left(\sum_{i=k+1}^{2 k-2} x_{i}^{2}\right)+2 k\left(\sum_{i=1}^{k} x_{i}\right)^{2}+4 k\left(\sum_{i=1}^{k} x_{i}\right)\left(\sum_{i=k+1}^{2 k-2} x_{i}\right) \\
& +2 k\left(\sum_{i=k+1}^{2 k-2} x_{i}\right)^{2}+4 k\left(\sum_{i=1}^{2 k-2} x_{i}\right) x_{2 k-1}+2 k x_{2 k-1}^{2}
\end{aligned}
$$




$$
\begin{aligned}
& =(2+k)\left(\sum_{i=1}^{k} x_{i}\right)^{2}+2 k\left(\sum_{i=k+1}^{2 k-2} x_{i}^{2}\right)+2 k\left(\sum_{i=1}^{k} x_{i}\right)\left(\sum_{i=k+1}^{2 k-2} x_{i}\right) \\
& +k\left(\sum_{i=k+1}^{2 k-2} x_{i}\right)^{2}+4 k\left(x_{2 k-1}+\frac{1}{2}\left(\sum_{i=1}^{2 k-2} x_{i}\right)\right)^{2} .
\end{aligned}
$$

The statement of the lemma is proved.

Remark 3.4.4 As $n \rightarrow \infty$, it follows that $m \rightarrow \infty$ as well and the asymptotic of option price is given by

$$
\begin{gathered}
\hat{C}_{T-m} \asymp S_{T-m} \sum_{\mathfrak{M}_{2 k}(m, p, x)} \frac{1}{(2 \pi)^{\frac{k}{2}}} \frac{1}{\sqrt{\left(\sum_{i=1}^{k} \bar{p}_{i}\right) \bar{p}_{k+1} \ldots \bar{p}_{2 k}}} \exp \left\{\bar{D}_{2 k-2}+\bar{R}_{2 k-1}\right\} \\
\quad-\frac{K}{\hat{r}^{m}} \sum_{\mathfrak{M}_{2 k}(m, \bar{p}, x)} \frac{1}{(2 \pi)^{\frac{k}{2}}} \frac{1}{\sqrt{\left(\sum_{i=1}^{k} p_{i}\right) p_{k+1} \ldots p_{2 k}}} \exp \left\{D_{2 k-2}+R_{2 k-1}\right\} .
\end{gathered}
$$

\subsection{Generalized Multinomial Black-Scholes op- tion pricing formula}

In the setting of asymptotic procedure, constructed in the section 3.2 we obtain the generalized multinomial Black-Scholes option price formula. We will show that the sequence of generalized multinomial CRR option prices $\hat{C}_{T-m, n}$ from (3.1.1) converges to some limit, as $n \rightarrow \infty$. This limit is a generalization of classical Black-Scholes option pricing formula [7] and depends on multinomiality parameters $c_{1}, \ldots, c_{k}$.

Theorem 3.5.1. The following convergence is valid for any dyadic $t \in[0, T]$

$$
\lim _{n \rightarrow \infty} \hat{C}_{T-m}=\hat{C}_{t}=S_{t} \Phi\left(f_{1}\left(S_{t}, T-t\right)\right)-K e^{-r(T-t)} \Phi\left(f_{2}\left(S_{t}, T-t\right)\right),
$$


where

$$
\begin{gathered}
f_{1}(s, t)=\frac{\ln \frac{s}{K}+(T-t) \frac{\ln c_{1} \ldots c_{k}+k}{k}\left(\frac{r}{k} \frac{\ln c_{1} \ldots c_{k}+k}{\prod_{i=1}^{k}\left(\ln c_{i}+1\right)}+\frac{\sigma^{2}}{2} \frac{\ln c_{1} \ldots c_{k}+k}{k}\right)}{\sigma \frac{\ln c_{1} \ldots c_{k}+k}{k} \sqrt{T-t}}, \\
f_{2}(s, t)=f_{1}(s, t)-\sigma \frac{\ln c_{1} \ldots c_{k}+k}{k} \sqrt{T-t}
\end{gathered}
$$

and $\Phi$ stands for the standard Gaussian cumulative distribution function $\Phi(x)=\frac{1}{\sqrt{2 \pi}} \int_{-\infty}^{x} e^{\frac{u^{2}}{2}} d u, \quad \forall x \in \mathbb{R}$.

Proof. Let $S_{t}=s$ be a value of the stock price at time $t$. First, we need to calculate limits of some quantities. Notice, that

$$
\lim _{n \rightarrow \infty}\left(1+r_{n}\right)^{-m_{n}}=\lim _{n \rightarrow \infty} e^{-m_{n} r \Delta_{n}}=e^{-r(T-t)} .
$$

Furthermore, for every $n>r^{2} \sigma^{-2} T$ we have

$$
d_{i, n}=\frac{1}{u_{i, n}}<\frac{1}{\hat{r}_{n}} \leq \hat{r}_{n}<u_{i, n}, \quad i=1, \ldots, k
$$

and as it was shown in the proof of lemma 3.4.3

$$
\lim _{n \rightarrow \infty} p_{1, n}=\ldots=\lim _{n \rightarrow \infty} p_{k, n}=\ldots=\lim _{n \rightarrow \infty} p_{2 k, n}=\frac{1}{2 k} .
$$

The following asymptotic holds true as $n \rightarrow \infty$.

$$
\begin{aligned}
& p_{i, n} \asymp \frac{1}{2 k}+\frac{\sqrt{\Delta_{n}}}{2 k \sigma\left(\ln c_{i}+1\right)}\left(r-\frac{\sigma^{2}\left(\ln c_{i}+1\right)^{2}}{2}\right), \quad i=1, \ldots, k \\
& p_{i, n} \asymp \frac{1}{2 k}-\frac{\sqrt{\Delta_{n}}}{2 k \sigma\left(\ln c_{i-k}+1\right)}\left(r-\frac{\sigma^{2}\left(\ln c_{i-k}+1\right)^{2}}{2}\right), \quad i=k+1, \ldots, 2 k .
\end{aligned}
$$

The limits of $\bar{p}_{i}, i=1, \ldots, 2 k$ are calculated in a similar way.

$$
\lim _{n \rightarrow \infty} \bar{p}_{1, n}=\lim _{n \rightarrow \infty} \frac{u_{1, n} p_{1, n}}{\hat{r}_{n}}=\frac{1}{2 k}
$$

and due to lemma 3.4 .3

$$
\lim _{n \rightarrow \infty} \bar{p}_{1, n}=\ldots=\lim _{n \rightarrow \infty} \bar{p}_{k}=\ldots=\lim _{n \rightarrow \infty} \bar{p}_{2 k}=\frac{1}{2 k} .
$$




\subsection{Generalized Multinomial Black-Scholes option pricing formula}

The asymptotic for $\bar{p}_{i, n}, i=1, \ldots, 2 k$ as $n \rightarrow \infty$ is given by

$$
\begin{aligned}
& \bar{p}_{i, n} \asymp \frac{1}{2 k}+\frac{\sqrt{\Delta_{n}}}{2 k \sigma\left(\ln c_{i}+1\right)}\left(r+\frac{\sigma^{2}\left(\ln c_{i}+1\right)^{2}}{2}\right), \quad i=1, \ldots, k \\
& \bar{p}_{i, n} \asymp \frac{1}{2 k}-\frac{\sqrt{\Delta_{n}}}{2 k \sigma\left(\ln c_{i-k}+1\right)}\left(r+\frac{\sigma^{2}\left(\ln c_{i-k}+1\right)^{2}}{2}\right), \quad i=k+1, \ldots, 2 k .
\end{aligned}
$$

Now we calculate the value of $a=a(S)=\inf \left\{j: D_{j, m}\left(S, c_{1}, \ldots, c_{k}\right) \neq \emptyset\right\}$.

$$
\begin{aligned}
& S \exp \left\{m_{1} \sigma\left(\ln c_{1}+1\right) \sqrt{\Delta_{n}}+\ldots+m_{k} \sigma\left(\ln c_{k}+1\right) \sqrt{\Delta_{n}}\right. \\
& \left.-m_{k+1} \sigma\left(\ln c_{1}+1\right) \sqrt{\Delta_{n}}-\ldots-m_{2 k} \sigma\left(\ln c_{k}+1\right) \sqrt{\Delta_{n}}\right\}=K
\end{aligned}
$$

From the conditions on the set $D_{j, m}\left(S, c_{1}, \ldots, c_{k}\right)$ it is known that $m_{1}+\ldots+m_{k}=j$. Since $m_{1}, \ldots, m_{k}$ are numbers of upward jumps with values $c_{1} u, \ldots, c_{k} u$ correspondingly and by assumptions $c_{1}, \ldots, c_{k}$ can occur equally likely, so that the number of jumps of different values should be approximately more or less the same, then we are entitled to suppose that $m_{1}=\ldots=m_{k}=\frac{j}{k}$ and respectively $m_{k+1}=\ldots=m_{2 k}=\frac{m-j}{k}$. Without loss of generality we consider $j$ and $m-j$ are divisible by $j$. So,

$$
\frac{j}{k}\left(\sum_{i=1}^{k} \ln c_{i}+k\right) \sigma \sqrt{\Delta_{n}}-\frac{m-j}{k}\left(\sum_{i=1}^{k} \ln c_{i}+k\right) \sigma \sqrt{\Delta_{n}}=\ln \frac{K}{S},
$$

therefore

$$
j=\frac{k\left(\ln \frac{K}{S}+\frac{m}{k}\left(\sum_{i=1}^{k} \ln c_{i}+k\right) \sigma \sqrt{\Delta_{n}}\right)}{2\left(\sum_{i=1}^{k} \ln c_{i}+k\right) \sigma \sqrt{\Delta_{n}}}
$$

or we write down it as

$$
j=\frac{\frac{k}{2} \ln \frac{K}{S}+\frac{m}{2}\left(\sum_{i=1}^{k} \ln c_{i}+k\right) \sigma \sqrt{\Delta_{n}}}{\left(\sum_{i=1}^{k} \ln c_{i}+k\right) \sigma \sqrt{\Delta_{n}}} .
$$

Since under such $j$ all conditions imposed in the definition of the set $D_{j, m}\left(S, c_{1}, \ldots, c_{k}\right) \quad$ (Definition 2.2.3) are fulfilled then $a=\frac{\frac{k}{2} \ln \frac{K}{S}+\frac{m}{2}\left(\sum_{i=1}^{k} \ln c_{i}+k\right) \sigma \sqrt{\Delta_{n}}}{\left(\sum_{i=1}^{k} \ln c_{i}+k\right) \sigma \sqrt{\Delta_{n}}}$. Now, due to the proposition 3.4.2 we have 


\subsection{Generalized Multinomial Black-Scholes option pricing formula}

to calculate the limit of the asymptotic expression of the option price value, $\breve{C}_{T-m}$ given by (3.4.5), as $n \rightarrow \infty$. Further, in order to shorten notations we will denote $p_{1}, \ldots, p_{2 k}, x_{1}, \ldots, x_{2 k}$ without subindex $n$ but it needs to be kept in mind that these quantities depend on $n$. Calculate

$$
\begin{gathered}
x_{2 k}=\frac{m_{2 k}-m p_{2 k}}{\sqrt{m}}=\frac{m-m_{1}-\ldots-m_{2 k-1}-m p_{2 k}}{\sqrt{m}} \\
=\frac{m-x_{1} \sqrt{m}-m p_{1}-\ldots-x_{2 k-1} \sqrt{m}-m p_{2 k-1}-m p_{2 k}}{\sqrt{m}}=-\left(x_{1}+\ldots+x_{2 k-1}\right) .
\end{gathered}
$$

From (3.4.7) we have that

$$
\begin{aligned}
\breve{C}_{T-m}= & S_{T-m} \sum_{\mathfrak{M}\left(m^{(2 k-2)}, \bar{p}, a\right)} \frac{1}{(\sqrt{2 \pi m})^{k-1}} \frac{1}{\sqrt{\left(\sum_{i=1}^{k} \bar{p}_{i}\right) \bar{p}_{2 k}}} \frac{1}{\sqrt{\prod_{i=k+1}^{2 k-2} \bar{p}_{i}}} \exp \left\{-\frac{1}{2} \bar{D}_{2 k-2}\right\} \overline{I S}_{2 k-1} \\
& -\frac{K}{\hat{r}^{m}} \sum_{\mathfrak{M}\left(m^{(2 k-2)}, p, a\right)} \frac{1}{(\sqrt{2 \pi m})^{k-1}} \frac{1}{\sqrt{\left(\sum_{i=1}^{k} p_{i}\right) p_{2 k}}} \frac{1}{\sqrt{\prod_{i=k+1}^{2 k-2} p_{i}}} \exp \left\{-\frac{1}{2} D_{2 k-2}\right\} I S_{2 k-1},
\end{aligned}
$$

where $m^{(2 k-2)}=m_{1}+\ldots+m_{2 k-2}$, since $x_{i} \in \mathbb{X}($ defined by $(3.4 .4)), i=1, \ldots, 2 k$, then

$$
\begin{aligned}
& \overline{I S}_{2 k-1}=\sum_{m_{2 k-1}=m \bar{p}_{2 k-1}-\bar{O}\left(m^{\gamma}\right)}^{m \bar{p}_{2 k-1}+\bar{O}\left(m^{\gamma}\right)} \frac{1}{\sqrt{2 \pi m}} \frac{1}{\sqrt{\bar{p}_{2 k-1}}} \exp \left\{-\frac{1}{2} \bar{R}_{2 k-1}\right\}, \\
& I S_{2 k-1}=\sum_{m_{2 k-1}=m p_{2 k-1}-\bar{O}\left(m^{\gamma}\right)}^{m p_{2 k-1}+\bar{O}\left(m^{\gamma}\right)} \frac{1}{\sqrt{2 \pi m}} \frac{1}{\sqrt{p_{2 k-1}}} \exp \left\{-\frac{1}{2} R_{2 k-1}\right\} .
\end{aligned}
$$

The sums $\overline{I S}_{2 k-1}$ and $I S_{2 k-1}$ are approximated by integrals.

$$
\begin{aligned}
I S_{2 k-1}= & \sum_{m_{2 k-1}}=m p_{2 k-1}-\bar{O}\left(m^{\gamma}\right) \\
& \frac{1}{\sqrt{2 \pi m}} \frac{1}{\sqrt{p_{2 k-1}}} \exp \left\{-\frac{1}{2} R_{2 k-1}\right\} \\
& \approx \int_{m p_{2 k-1}-\bar{O}\left(m^{\gamma}\right)}^{m p_{2 k-1}+\bar{O}\left(m^{\gamma}\right)} \frac{1}{\sqrt{2 \pi m}} \frac{1}{\sqrt{p_{2 k-1}}} e^{-\frac{1}{2}\left[4 k\left(\frac{m_{2 k-1}-m p_{2 k-1}}{\sqrt{m}}+\frac{1}{2}\left(\sum_{i=1}^{2 k-2} x_{i}\right)\right)^{2}\right]} d m_{2 k-1}
\end{aligned}
$$




\subsection{Generalized Multinomial Black-Scholes option pricing formula}

$$
\begin{gathered}
=\int_{-\bar{O}\left(m^{\gamma-\frac{1}{2}}\right)}^{\bar{O}\left(m^{\gamma-\frac{1}{2}}\right)} \frac{1}{\sqrt{2 \pi}} \frac{1}{\sqrt{p_{2 k-1}}} e^{-\frac{1}{2}\left[4 k\left(y_{2 k-1}+\frac{1}{2}\left(\sum_{i=1}^{2 k-2} x_{i}\right)\right)^{2}\right]} d y_{2 k-1} \\
\quad=\int_{a_{n}}^{b_{n}} \frac{1}{\sqrt{2 \pi}} \frac{1}{\sqrt{4 k p_{2 k-1}}} e^{-\frac{1}{2} u_{2 k-1}^{2}} d u_{2 k-1}=\frac{1}{\sqrt{2}}\left(\Phi\left(b_{n}\right)-\Phi\left(a_{n}\right)\right),
\end{gathered}
$$

where in last two equalities we applied the substitutions of variables $y_{2 k-1}=\frac{m_{2 k-1}-m p_{2 k-1}}{\sqrt{m}}$ and $u_{2 k-1}=\sqrt{4 k}\left(y_{2 k-1}+\frac{1}{2}\left(\sum_{i=1}^{2 k-2} x_{i}\right)\right)$. The integration limits $a_{n}$ and $b_{n}$ are given by

$$
\begin{aligned}
& a_{n}=\sqrt{4 k}\left(-\bar{O}\left(m^{\gamma-\frac{1}{2}}\right)+\frac{1}{2}\left(\sum_{i=1}^{2 k-2} x_{i}\right)\right), \\
& b_{n}=\sqrt{4 k}\left(\bar{O}\left(m^{\gamma-\frac{1}{2}}\right)+\frac{1}{2}\left(\sum_{i=1}^{2 k-2} x_{i}\right)\right) .
\end{aligned}
$$

Since $x_{i} \in \mathbb{X}$ (the set $\mathbb{X}$ defined by (3.4.4)), $i=1, \ldots, 2 k$, then $\left|\sum_{i=1}^{2 k-2} x_{i}\right| \approx \bar{O}\left(m^{\frac{1}{2}-\beta}\right)$, where $\frac{1}{3}<\beta<\frac{1}{2}$ and from (3.4.3) we know that $\frac{1}{2}<\gamma<\frac{2}{3}$, then it is clear that both $0<\frac{1}{2}-\beta<\frac{1}{6}$ and $0<\gamma-\frac{1}{2}<\frac{1}{6}$. So that both summands in the expressions for $a_{n}$ and $b_{n}$ are of the same order. There are now two possibilities which provide the conditions when $a_{n} \rightarrow-\infty$ and $b_{n} \rightarrow+\infty$ :

(1) if $\sum_{i=1}^{2 k-2} x_{i}>0$, then it must be $\sum_{i=1}^{2 k-2} x_{i}<2 \bar{O}\left(m^{\gamma-\frac{1}{2}}\right)$,

(2) if $\sum_{i=1}^{2 k-2} x_{i}<0$, then it must be $\sum_{i=1}^{2 k-2} x_{i}>-2 \bar{O}\left(m^{\gamma-\frac{1}{2}}\right)$.

Therefore, it follows that the sum $\sum_{i=1}^{2 k-2} x_{i}$ satisfies these conditions (1) and (2) if and only if

$$
\left|\sum_{i=1}^{2 k-2} x_{i}\right|<\bar{O}\left(m^{\gamma-\frac{1}{2}}\right),
$$

which is equivalent to the conditions on $m_{1}, \ldots, m_{k}, m_{k+1}, \ldots, m_{2 k-2}$ defined in (3.4.3) and there are no contradictions between that what we need and what is assumed. Under these conditions, $b_{n} \rightarrow+\infty$ and $a_{n} \rightarrow-\infty$, as $n \rightarrow \infty$. Therefore,

$$
I S_{2 k-1} \approx \frac{1}{\sqrt{2}}, \quad \text { as } \quad n \rightarrow \infty
$$




\subsection{Generalized Multinomial Black-Scholes option pricing formula}

If $k>2$ then, $D_{2 k-2}, \bar{D}_{2 k-2}$ can be transformed into $D_{2 k-3}$ and $\bar{D}_{2 k-3}$ correspondingly, by extracting the $x_{2 k-2}$ and establishing the corresponding $R_{2 k-2}$ and $\bar{R}_{2 k-2}$. This process can be continued until $D_{k}, \bar{D}_{k}, R_{k+1}$ and $\bar{R}_{k+1}$ are obtained. Consequently, the number of sums in the option price formula (3.4.7) will be reduced. There are $k-2$ steps when it is possible to calculate the limit of approximating integral. During next following steps the approximation is given by

$$
\begin{gathered}
\sum_{m_{2 k-2}=m p_{2 k-2}-\bar{O}\left(m^{\gamma}\right)}^{m p_{2 k-2}+\bar{O}\left(m^{\gamma}\right)} \frac{1}{\sqrt{2 \pi m}} \frac{1}{\sqrt{p_{2 k-2}}} \exp \left\{-\frac{1}{2} R_{2 k-2}\right\} \approx \frac{\sqrt{2}}{\sqrt{3}}, \quad \text { as } n \rightarrow \infty \\
\sum_{m_{2 k-3}=m p_{2 k-3}-\bar{O}\left(m^{\gamma}\right)}^{m p_{2 k-3}+\bar{O}\left(m^{\gamma}\right)} \frac{1}{\sqrt{2 \pi m}} \frac{1}{\sqrt{p_{2 k-3}}} \exp \left\{-\frac{1}{2} R_{2 k-3}\right\} \approx \frac{\sqrt{3}}{\sqrt{4}}, \quad \text { as } n \rightarrow \infty \\
\vdots \\
\sum_{m p_{k+2}+\bar{O}\left(m^{\gamma}\right)} \frac{1}{\sqrt{2 \pi m}} \frac{1}{\sqrt{p_{k+2}}} \exp \left\{-\frac{1}{2} R_{k+2}\right\} \approx \frac{\sqrt{k-2}}{\sqrt{k-1}}, \quad \text { as } n \rightarrow \infty
\end{gathered}
$$

and the $(k-2)$ th approximation is given by

$$
\sum_{m_{k+1}=m p_{k+1}-\bar{O}\left(m^{\gamma}\right)}^{m p_{k+1}+\bar{O}\left(m^{\gamma}\right)} \frac{1}{\sqrt{2 \pi m}} \frac{1}{\sqrt{p_{k+1}}} \exp \left\{-\frac{1}{2} R_{k+1}\right\} \approx \frac{\sqrt{k-1}}{\sqrt{k}}, \quad \text { as } \quad n \rightarrow \infty .
$$

So that it is left to estimate

$$
\begin{aligned}
& \frac{1}{\sqrt{k}} \sum_{m_{1}+\ldots+m_{k}=a}^{m\left(p_{1}+\ldots+p_{k}\right)+\bar{O}\left(m^{\gamma}\right)} \frac{1}{\sqrt{2 \pi m}} \frac{1}{\sqrt{\left(p_{1}+\ldots+p_{k}\right) p_{2 k}}} \exp \left\{-\frac{1}{2} D_{k}\right\} \\
& =\frac{1}{\sqrt{k}} \sum_{\sum_{1}+\ldots+m_{k}=a}^{m\left(p_{1}+\ldots+p_{k}\right)+\bar{O}\left(m^{\gamma}\right)} \frac{1}{\sqrt{2 \pi m}} \frac{1}{\sqrt{\left(p_{1}+\ldots+p_{k}\right) p_{2 k}}} e^{-\frac{1}{2}\left[4\left(\sum_{i=1}^{k} x_{i}\right)^{2}\right]} \\
& \approx 2 \int_{a}^{m\left(p_{1}+\ldots+p_{k}\right)+\bar{O}\left(m^{\gamma}\right)} \frac{1}{\sqrt{2 \pi m}} e^{-\frac{1}{2}\left[4\left(\frac{m_{1}+\ldots+m_{k}-m\left(p_{1}+\ldots+p_{k}\right)}{\sqrt{m}}\right)^{2}\right]} d\left(m_{1}+\ldots+m_{k}\right)
\end{aligned}
$$




\subsection{Generalized Multinomial Black-Scholes option pricing formula}

$$
\begin{gathered}
=2 \int_{\frac{a-m\left(p_{1}+\ldots+p_{k}\right)}{\sqrt{m}}}^{\bar{O}\left(m^{\gamma-\frac{1}{2}}\right)} \frac{1}{\sqrt{2 \pi}} e^{-\frac{1}{2}\left(4 y_{1}^{2}\right)} d y_{1}=\int_{\frac{2\left(a-m\left(p_{1}+\ldots+p_{k}\right)\right)}{\sqrt{m}}}^{2 \bar{O}\left(m^{\gamma-\frac{1}{2}}\right)} \frac{1}{\sqrt{2 \pi}} e^{-\frac{1}{2} u_{1}^{2}} d u_{1} \\
=\Phi\left(2 \bar{O}\left(m^{\gamma-\frac{1}{2}}\right)\right)-\Phi\left(\frac{2\left(a-m\left(p_{1}+\ldots+p_{k}\right)\right)}{\sqrt{m}}\right),
\end{gathered}
$$

where the variables substitution has been applied twice, first one is $y_{1}=\frac{m_{1}+\ldots+m_{k}-m\left(p_{1}+\ldots+p_{k}\right)}{\sqrt{m}}$ and the second one is $u_{1}=2 y_{1}$. Now remember the expression for $a$ and calculate the limit

$$
\begin{array}{r}
f_{2}(S, T-t):=-\lim _{n \rightarrow \infty} \frac{2\left(a-m\left(p_{1}+\ldots+p_{k}\right)\right)}{\sqrt{m}}=-\lim _{n \rightarrow \infty} \frac{k \ln \frac{K}{S}+\sigma\left(\sum_{i=1}^{k} \ln c_{i}+k\right) m \sqrt{\Delta_{n}}}{\sigma\left(\sum_{i=1}^{k} \ln c_{i}+k\right) \sqrt{m} \sqrt{\Delta_{n}}} \\
-\lim _{n \rightarrow \infty} \frac{2 \sigma\left(\sum_{i=1}^{k} \ln c_{i}+k\right) m \sqrt{\Delta_{n}}\left(\frac{1}{2}+\frac{r \sqrt{\Delta_{n}}}{2 k \sigma}\left(\sum_{i=1}^{k} \frac{1}{\ln c_{i}+1}\right)-\frac{\sigma^{2} \sqrt{\Delta_{n}}}{2 k \sigma}\left(\sum_{i=1}^{k} \frac{\ln c_{i}+1}{2}\right)\right)}{\sigma\left(\sum_{i=1}^{k} \ln c_{i}+k\right) \sqrt{m} \sqrt{\Delta_{n}}} \\
=-\frac{k \ln \frac{K}{S}-(T-t) \frac{\left(\ln c_{1} \ldots c_{k}+k\right)}{k}\left(r \frac{\left(\ln c_{1} \ldots c_{k}+k\right)}{\left(\ln c_{1}+1\right) \ldots\left(\ln c_{k}+1\right)}-\frac{\sigma^{2}}{2}\left(\ln c_{1} \ldots c_{k}+k\right)\right)}{\sigma\left(\ln c_{1} \ldots c_{k}+k\right) \sqrt{T-t}} \\
=\frac{\ln \frac{S}{K}+(T-t) \frac{\left(\ln c_{1} \ldots c_{k}+k\right)}{k}\left(\frac{r}{k} \frac{\left(\ln c_{1} \ldots c_{k}+k\right)}{\left(\ln c_{1}+1\right) \ldots\left(\ln c_{k}+1\right)}-\frac{\sigma^{2}}{2} \frac{\left(\ln c_{1} \ldots c_{k}+k\right)}{k}\right)}{\sigma \frac{\left(\ln c_{1} \ldots k_{k}+k\right)}{k} \sqrt{T-t}} .
\end{array}
$$

Analogously the limit of $\frac{2\left(a-m\left(\bar{p}_{1}+\ldots+\bar{p}_{k}\right)\right)}{\sqrt{m}}$ as $n \rightarrow \infty$ has been calculated. It is equal to

$$
f_{1}(S, T-t)=\frac{\ln \frac{S}{K}+(T-t) \frac{\left(\ln c_{1} \ldots c_{k}+k\right)}{k}\left(\frac{r}{k} \frac{\left(\ln c_{1} \ldots c_{k}+k\right)}{\left(\ln c_{1}+1\right) \ldots\left(\ln c_{k}+1\right)}+\frac{\sigma^{2}}{2} \frac{\left(\ln c_{1} \ldots c_{k}+k\right)}{k}\right)}{\sigma \frac{\left(\ln c_{1} \ldots c_{k}+k\right)}{k} \sqrt{T-t}},
$$

and has a relation to $f_{2}$ as $f_{2}(S, T-t)=f_{1}(S, T-t)-\sigma \frac{\left(\ln c_{1} \ldots c_{k}+k\right)}{k} \sqrt{T-t}$

The theorem is proved.

Remark 3.5.2 We obtain classical Black-Scholes option pricing formula from (3.5.1) if it is assumed that $k=1$ and $c_{1}=1$. 


\section{Chapter 4}

\section{Numerical Results}

In this chapter we describe some numerical results. First, it is shown that generalized model (1.1.2) of the stock price process can give better approximation to real data then binomial model. It occurs when the multinomiality parameters $c_{1}, \ldots, c_{k}$ are embedded into the generalized stock price model.

We propose simple multinomiality parameters estimation procedure and give examples of data approximation using the new stock price model with two multinomiality parameters $c_{1}$ and $c_{2}$. When the generalized stock price model with multinomiality parameters is used then we say that there is a multinomial approximation, when the classical binomial stock price model is used, then we say there is a binomial approximation. The embedding of only two multinomiality parameters gives much better results with respect to corresponding measures of approximation which are mean squared error and mean absolute error.

The mean squared errors obtained under multinomial approximation are much smaller than those obtained under binomial one. The comparison is carried out using the real data of stocks. The results of these empirical studies also suggest when the multinomiality parameters should be included into stock price modelling and when it is worthless, so that one should use the binomial model which is easier. Correspondingly, we calculate the multinomial option prices using the formula (2.3.8) and see how the multinomiality parameters influence on the option price. 


\subsection{Stock Price Modelling. Hull-White Algorithm}

In order to simulate the stock price process using the generalized model established in Chapter 1 by (1.1.2)

$$
S_{t}=S_{t-1} X_{t-1} \nu_{t-1}, \quad \forall \quad t \leq T,
$$

provided that the values of $\left(X_{t}\right)_{t \leq T}$ are contained in the multinomiality set $\mathbb{C}_{k}$ defined by (2.2.1), one has to be able to calculate the parameters $u$ and $d$ as well as $c_{1}, \ldots, c_{k}$ from real data. Intuitively, it is clear that these parameters are dependent on the variance $\sigma$ and mean $\mu$ of the data. So that first, one should estimate the parameters $\sigma$ and $\mu$ and then all others. The estimation of $\sigma$ and $\mu$ is standard estimation of variance and mean.

There are several methods for estimation of $u$ and $d$. We describe the simple one which is frequently used to model stock returns.

\section{Hull-White (HW) Algorithm ( [27])}

We set $p=\frac{1}{2}$ and determine $u$ and $d$ from the following equations

$$
\begin{aligned}
& \text { (1) } \frac{u+d}{2}=1+\mu \Delta t \\
& \text { (2) } u-d=2 \sigma \sqrt{\Delta t}
\end{aligned}
$$

where $\mu$ is the drift parameter or the mean, measures the average percent change in the stock price over the time; $\sigma$ is the volatility parameter or the variance, measures the randomness of the stock price; $\Delta t$ is a time period after which we regard the stock price. We assume

$$
S_{i}=\nu_{i-1} S_{i-1}, \quad i=1, \ldots, n,
$$

where $\left(\nu_{k}\right)_{k \leq n}$ are independent Bernoulli random variables, such that

$$
\mathbb{P}\left\{\frac{S_{i}}{S_{i-1}}=u\right\}=\mathbb{P}\left\{\frac{S_{i}}{S_{i-1}}=d\right\}=\frac{1}{2} .
$$

The estimates for $\mu \Delta t$ and $\sigma^{2} \Delta t$ are

$$
\bar{U}=\frac{1}{n} \sum_{i=0}^{n-1}\left(\nu_{i}-1\right)=\frac{1}{n} \sum_{i=1}^{n}\left(\frac{S_{i}}{S_{i-1}}-1\right)
$$


and

$$
s^{2}=\frac{1}{n-1}\left(\sum_{i=1}^{n}\left(\frac{S_{i}}{S_{i-1}}-1\right)^{2}-n \bar{U}^{2}\right) .
$$

The numbers $\bar{U}$ and $s^{2}$ are the sample mean and variance that are determined from the real stock price data $S_{1}, S_{2}, \ldots, S_{n}$. The estimates for $\mu$ and $\sigma$ are obtained as

$$
\mu \approx \frac{\bar{U}}{\Delta t}, \quad \sigma \approx \frac{s}{\Delta t} .
$$

Solving (1) and (2) form (4.1.1) we obtain that

$$
\begin{aligned}
& u=1+\mu \Delta t+\sigma \sqrt{\Delta t} \\
& d=1+\mu \Delta t-\sigma \sqrt{\Delta t}
\end{aligned}
$$

\subsection{Stock Price Modelling. Estimation of multi- nomiality parameters}

It is assumined that multinomiality parameters $c_{1}, \ldots, c_{k}$ occur equally likely with probabilities $\frac{1}{k}$ (see section 2.2). Under these assumptions we propose to use the following algorithm to estimate parameters $c_{1}, \ldots, c_{k}$ from real data.

Hull-White-Kan algorithm. The Estimation of Parameters $c_{1}, \ldots, c_{k}$ First, we assume that only parameter $c_{1}$ is include in the model of stock price, then

$$
\frac{(u+d)\left(1+c_{1}\right)}{4}=1+\mu \Delta t
$$

then

$$
c_{1}=\frac{4(1+\mu \Delta t)}{u+d}-1
$$

The stock price model with one parameter $c_{1}$ implicates the classical binomial stock price model when $c_{1}=0$.

If, due to real data, the embedding of one parameter $c_{1}$ is not satisfactory, then we include in the model parameter $c_{2}$, which is defined as

$$
c_{2}=2-c_{1}
$$

we continue to determine $c_{3}$ as a solution of the equation

$$
\frac{(u+d)\left(1+c_{1}+c_{2}+c_{3}\right)}{8}=1+\mu \Delta t
$$


then

$$
c_{3}=\frac{8(1+\mu \Delta t)}{u+d}-3 .
$$

Next value of $c_{4}$ we define as

$$
c_{4}=2-c_{3}
$$

The procedure can be continued like that until it is necessary.

One can see that the combination of the model (1.1.2) given $\left(X_{t}\right)_{t \leq T} \in \mathbb{C}_{k}$ and of the equation (1) in HW algorithm have been used to construct the multinomiality parameters $c_{1}, \ldots, c_{k}$ estimation procedure.

\subsection{The Stock Price Modelling. Simulations}

We consider several raw financial data sets that have been observed during some time period, mostly these are the stock prices of different German companies. The data were downloaded from the web site www.comdirect.de and identified with so called WKN number. We consider the data samples of size $n=500$. Also, the data sets are classified by the value of historical volatility. More precisely, we consider the data sets which have volatility $\sigma<35 \%, 35 \%<\sigma<65 \%$, $65 \%<\sigma<100 \%$ and when $\sigma>100 \%$.

Using the binomial model and the new multinomial one we want to fit the model simulated data to the raw data and calculate the corresponding mean squred error, which is defined as the averaged squared difference between modelled and real observations. 


\section{Volatility $\sigma=11 \%$}
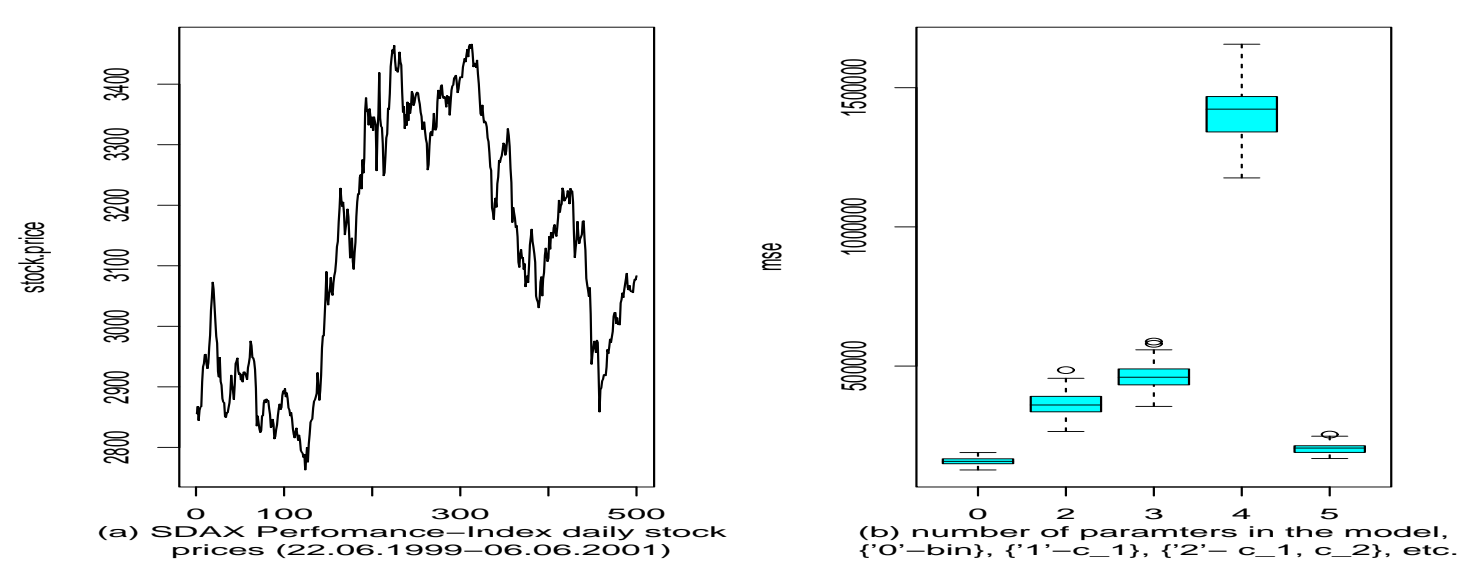

Figure 4.3.1: (a) The stock prices of SDAX Performance Index with volatility for the period of 500 trading days $\sigma=11 \%$. (b) The mean squared errors boxplots obtained using different models.

On the Figure 4.3.1 (a) one can see the low volatile data ( $\sigma=11 \%$ ) of SDAX Performance Index daily stock prices, on the Figure 4.3.1 (b) the boxplots of mean squared errors obtained when different multinomial models have been used to approximate stock price data are represented. The first boxplot marked ' 0 ' is the boxplot of mean squared errors of binomial approximation, further, the marks ' 2 ', ' 3 ', ... mean that the multinomial models with $c_{1}, c_{2}, c_{1}, c_{2}, c_{3}$, and so on multinomiality parameters have been used for approximation. It is clear from the picture that the application of multinomial model with different number of multinomiality parameters does not show better approximation. When the market volatility is low, there are no much fluctuations and frictions, then it is better to use the binomial model of stock price to fit real data.

Further, we will consider the data set with higher volatility and it will be shown that in those cases the multinomial approximation works better than the binomial one.

We will see that the introduction of multinomiality parameters improves the data approximation the market is not stable and can express some spikes than the multinomiality parameters allow us to model that better. 


\section{Volatility $\sigma=49 \%$}
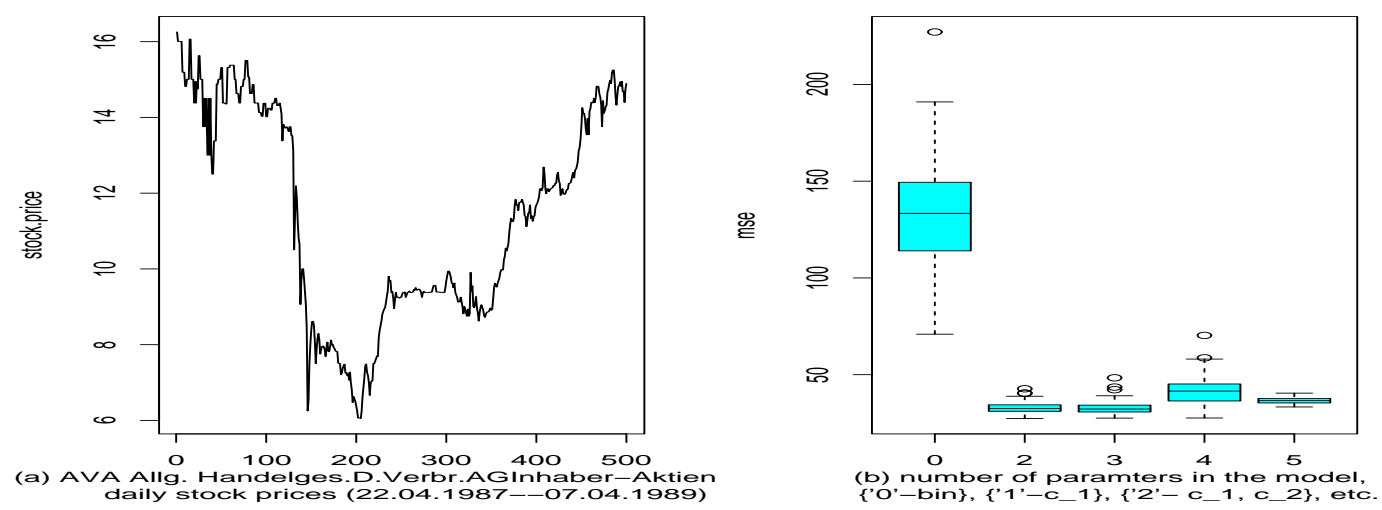

Figure 4.3.2: (a) The stock prices of AVA ALLC. HANDELSGES. D. VERBR. AGInhaber-Aktien o. N. with volatility for the period of 500 trading days $\sigma=49 \%$. (b) The mean squared errors boxplots obtained using different models.

\section{Volatility $\sigma=91 \%$}
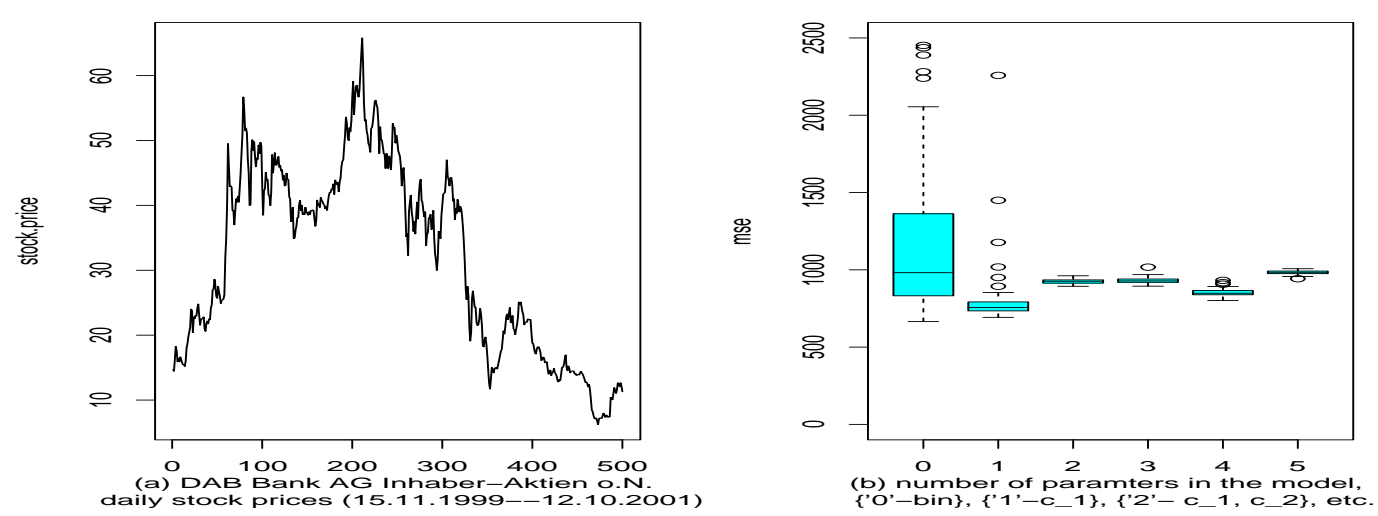

Figure 4.3.3: (a) The stock prices of DAB BANK AGInhaber-Aktien o. N. with volatility for the period of 500 trading days $\sigma=91 \%$. (b) The mean squared errors boxplots obtained using different models. 


\section{Volatility $\sigma=131 \%$}
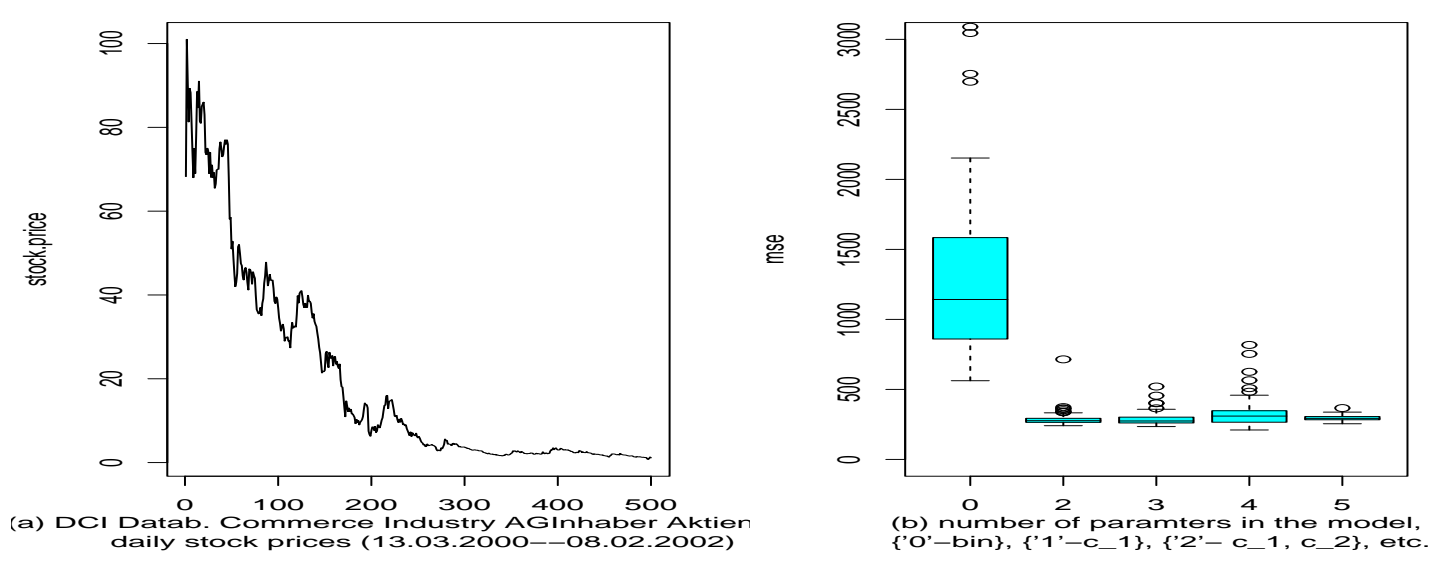

Figure 4.3.4: (a) The stock prices of DCI DATAB COMMERCE INDUSTRY AGInhaber-Aktien o. N. with volatility for the period of 500 trading days $\sigma=131 \%$. (b) The mean squared errors boxplots obtained using different models.

From figures 4.3.2, 4.3.3 and 4.3.4 we can see that the data AVA ALLC. HANDELSGES. D. VERBR. AGInhaber-Aktien o. N., DAB BANK AGInhaberAktien o. N. and DCI DATAB. COMMERCE INDUSTRY AGInhaber-Aktien o. N. show up that the multinomial model of stock price process is better than the binomial model of stock price process. It gives smaller mean squared errors and therefore, the data approximation is better.

All these data sets have volatility higher than 35\%, which indicates that in these cases the market is more unsettled. And this must serve as a signal to apply the multinomial model to simulate stock prices. However, there is a problem to determine how many multinomiality parameters have to be considered in order to obtain sufficiently good results and not to have overcomplicated calculations.

From Figure 4.3.3, one can see that it is sufficient to use the multinomial model with just one parameter $c_{1}$ to obtain significantly better results of data simulation. But sometimes this is not so and the problem of defining the 


\subsection{Numerical option price calculation. Multinomial CRR Model}

number of parameters in the multinomial model is also related to the problem of parameters estimation.

However, it is clear, that the multinomial modelling gives significantly better results for the approximation of highly volatile data.

\subsection{Numerical option price calculation. Multino- mial CRR Model}

We use the multinomial CRR model (2.3.8) in order to calculate the option price and to compare it with the Option Payoff $\left(S_{T}-K\right)^{+}(T$ is expiry date and $\mathrm{K}$ is a strike price) and option price obtained using the classical binomial CRR model [20].

We will consider examples with data sets of high volatility, i.e. AVA ALLC. HANDELSGES. D. VERBR. AGInhaber-Aktien o. N. (Figure 4.3.2), DAB BANK AGInhaber-Aktien o. N. (Figure 4.3.3) and DCI DATAB. COMMERCE INDUSTRY AGInhaber-Aktien o. N. (Figure 4.3.4), since they are approximated better with multinomial model. We expect that the option price calculated using multinomial CRR option price formula (2.3.8) with parameters $c_{1}$ and $c_{2}$ will give the price value which is closer to the Option Payoff than the value of option price obtained using binomial CRR formula.

To use the multinomial CRR (2.3.8) we have to know the parameters $c_{1}$ and $c_{2}$, for that we use the estimation procedure (HWK algorithm) proposed in the section 4.2. One can take arbitrarily any two parameters from the sequence of $c_{1}, \ldots, c_{k}$ obtained by the estimation procedure. We should notice that the multinomiality parameters values by itself sometimes don't give good results but if to consider some range of these parameters, i.e. $c_{1}+\delta, \ldots, c_{k}+\delta$, where $|\delta|$ can be small as hundredth or of smaller order, then it is possible to obtain sufficiently good results.

In the following numerical examples we will always consider that expiration time is $T=500$, the time before the expiration is equal to $m=100$, which means that we estimate the option price at time $t=400,100$ days before the expiration. Since we use the option price formula (2.3.8) we need to know the 
number $n_{1}$ of parameters $c_{1}$ occurred from time $t=T-m(t=400$ in our case) until the expiry date $t=T$. The corresponding number $n_{2}$ of parameters $c_{2}$ is equal to $n_{2}=m-n_{1}$. We consider that $n_{1}=50$ in order to define suitable $c_{1}$ and $c_{2}$, then we observe the option price when $n_{1}$ and $n_{2}$ change and see how the multinomial option price changes so that we can define the optimal for the option price values of $n_{1}$ and $n_{2}$.

For the calculation of binomial CRR option price [20] and multinomial CRR option price (2.3.8) written on the same stock, we use the same values of $T, S_{T}, K, s, r$ and $m$. So that we can compare them with the option payoff and see which model of option price gives values closer to this payoff. 
Example 4.4.1 (AVA ALLG. HANDELGES. D. VERBR. AGInhaber-Aktien Figure 4.3.2 (a))

We consider the interest rate as $r=0.01$, the stock price at time $t=400$ is equal to 11.6883 (i.e. $s=S_{400}=11.6883$ ). Since the stock price at time $T=500$ is equal to 14.8954 (i.e. $S_{500}=14.8954$ ), then we put the strike price of the call option as $K=8$, so that the option is in-the-money option, which means that it is beneficial for a buyer to exercise the option. Consequently, the option payoff is equal to $S_{T}-K=6.8954$. Since the volatility of the data is high enough, $\sigma=49 \%$ then it is suggested to use the CRR multinomial model for option pricing.
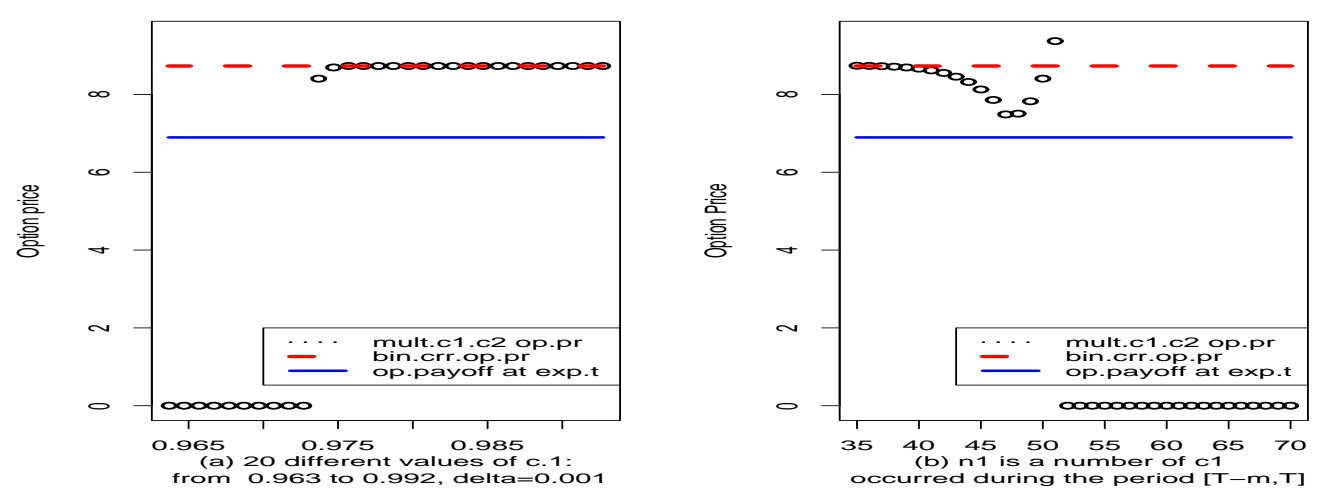

Figure 4.4.1: (a) The option price in dependence on the values of the parameter c1 (dotted line), binomial CRR option price (dashed line), option payoff at expiry time (solid line). (b) The option price in dependence on the $n_{1}$, number of parameters $c_{1}$, occurred from time $t=T-m$ until expiry date $t=T$.

One can see on the Figure 4.4.1 (a) that there is one value of $c_{1}$ where the multinomial CRR option price dependent on $c_{1}$ is closer to the option payoff than the binomial CRR option price. We determine this value of $c_{1}$ as $c_{1}^{*}$ and use it to consider further the dependence of multinomial CRR option price on the number of the parameters $c_{1}^{*}$ occurred from $t=400$ until the expiration time $T=500$. Under zero-values we understand the non-informative results and we do not consider these values of $n 1$. It is shown (Figure 4.4.1 (b)) that when $n 1=47$ and $n 1=48$ the multinomial CRR option price (dotted line) is closer to the option payoff than the binomial CRR option price which is constant. 


\subsection{Numerical option price calculation. Multinomial CRR Model}

Example 4.4.2 (DAB BANK AG Inhaber-Aktien,Figure 4.3.3 (a))

We consider the interest rate as $r=0.01$, the stock price at time $t=400$ is equal to 18.75 (i.e. $s=S_{400}=18.75$ ). Since the stock price at time $T=500$ is equal to 11.3 (i.e. $S_{500}=11.3$ ), then we put the strike price of the call option as $K=8$, so that the option is in-the-money option, which means that it is beneficial for a buyer to exercise the option. Consequently, the option payoff is equal to $S_{T}-K=3.3$. The historical volatility of the data is equal to $91 \%$. It is shown on the Figure 4.3.2 (b) that these stock prices are better modelled by multinomial model. We will see how the option price is changed when the multinomial CRR model is used.
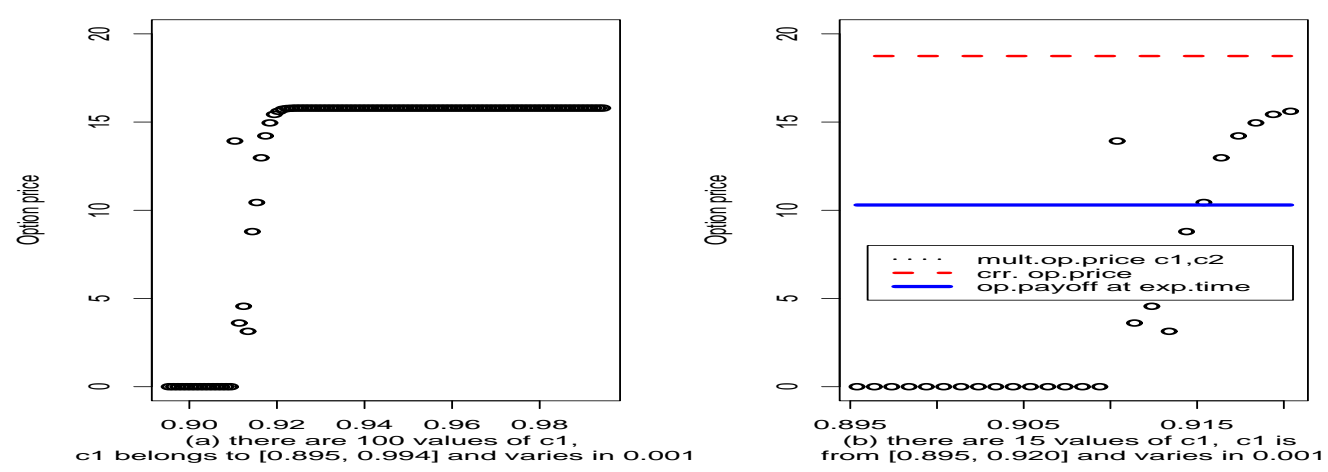

Figure 4.4.2: (a) The multinomial CRR option price in dependence on the values of parameter c1. (b) The multinomial CRR option price in dependence on the values of parameter c1 (dotted line), binomial CRR option price (dashed line) and the option payoff (solid line).

On the Figure 4.4.2 (a) the changes of the multinomial option price in dependence on the parameter $c 1$ are shown. One can see that there are some values of $c 1$ which influence on the option price significantly.

From the Figure 4.4.2 (b) one can see that there is a value of $\mathrm{c} 1$ which provides the value of multinomial CRR option price which is very close to the option payoff. So that there is no necessity to consider the dependence of option price from the number of $c 1$ occurred. These values of multinomiality parameter $c 1$ are $0.913,0.912,0.911$ under which the option price is equal to $3.138,4.558,3.609$ correspondingly and these values are very close to the option payoff which is equal to 3.3 . 


\subsection{Numerical option price calculation. Multinomial CRR Model}

\section{Example 4.4.3 (DCI DATAB.COMMERCE INDUSTRY AG}

\section{Inhaber-Aktien o.N.,Figure 4.3.2 (a))}

We consider the interest rate as $r=0.05$, the stock price at time $t=400$ is equal to 3 (i.e. $s=S_{400}=3$ ). Since the stock price at time $T=500$ is equal to 1.13 (i.e. $S_{500}=1.13$ ), then we put the strike price of the call option as $K=1$, so that the option is in-the-money option, which means that it is beneficial for a buyer to exercise the option. Consequently, the option payoff is equal to $S_{T}-K=0.13$. The data in this example have high volatility, $\sigma=131 \%$.

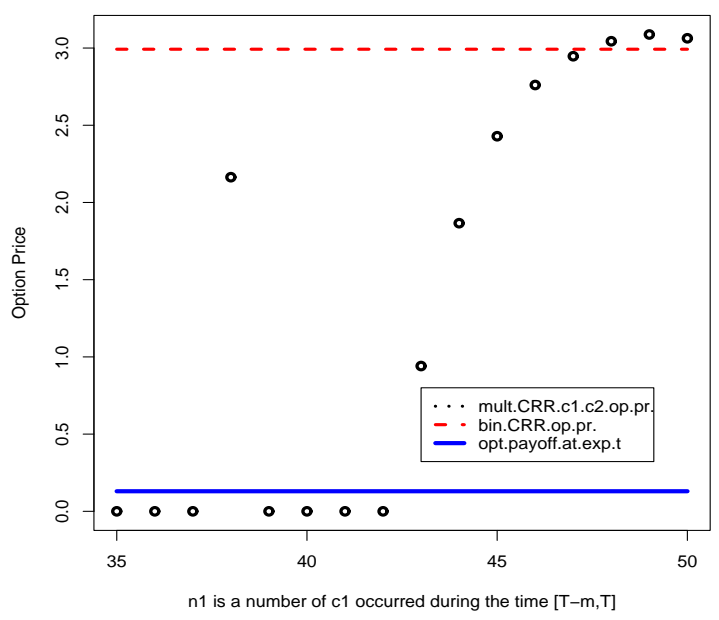

Figure 4.4.3: Multinomial CRR option price in dependence on the $n_{1}$, number of parameters $c 1$, occurred during $[T-m, T]$ is given by dotted line, binomial CRR option price is dashed one. The option payoff is solid line.

As one can see there are some values of $n_{1}$ when the multinomial CRR option price in dependence on $n_{1}$ yields the option prices which are closer to the option payoff than the binomial CRR option price. So that the multinomial CRR option price model for this data set allows produce better results to estimate the option price at time $t=T-m$. 


\subsection{Generalized multinomial Black-Scholes for- mula. Simulations}

In this section we consider the option prices calculated by means of the classical Black-Scholes (BS) formula [7] and the option prices calculated by means of the generalized multinomial Black-Scholes (MBS) option pricing formula (3.5.1). It is noticed, that multinomiality parameters $c_{1}, \ldots, c_{k}$ influence rather on option prices for stocks with high volatility than for stocks with low volatility. So that it is suggested to apply the multinomial Black-Scholes for option pricing when the market is of high volatility. We will stick to the data sets used in previous sections to show how the multinomial Black-Scholes formula depends on multinomiality parameters $c_{1}, \ldots, c_{k}$.

Example 4.5.1 ( AVA Allg. Handelges.D.Verbr. AGInhaber-Aktien Figure 4.3.2 (a))

We calculate the multinomial BS option price in dependence on parameters $c_{1}$ and $c_{2}$ and consider the strike price $K=8$, interest rate $r=0.01$, the current stock price $s=S_{400}$, time $\tau=\frac{100}{253}$.

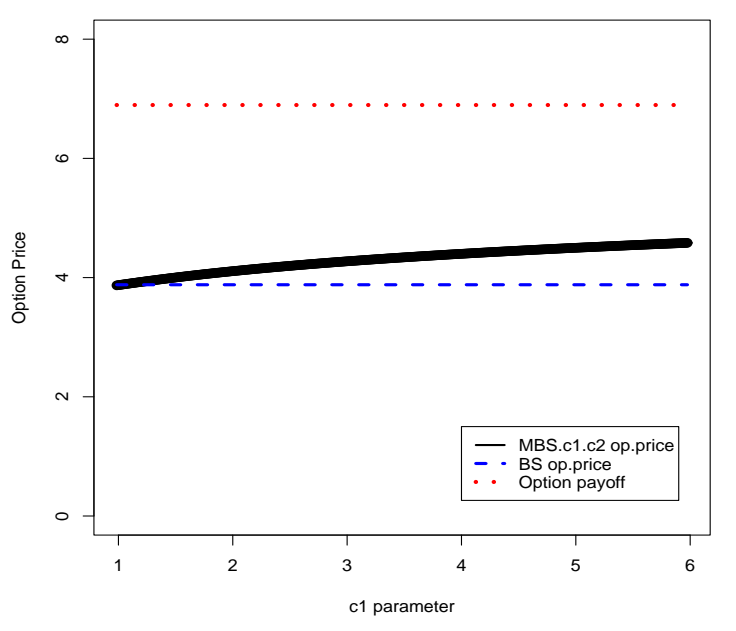

Figure 4.5.1: Multinomial Black-Scholes option price in dependence on the parameter $c 1$ (solid line) while the parameter $c_{2}$ is fixed and $c_{2}=0.978$, option payoff (dotted line) and classical Black-Scholes option price (dashed line). 


\subsection{Generalized multinomial Black-Scholes formula. Simulations}

It is shown on the Figure 4.5.1 that the option price obtained using multinomial Black-Scholes (MBS) increases and approaches to the option payoff when the parameter $c 1$ increases. It is clear, that adjusting properly the value of parameter $c 1$ it is possible to obtain the option prices closer to the option payoff.

We should notice that there is no preferences which parameter to assign to be fixed and which to use as adjusting the option price parameter. With the same success one can use parameter $c_{2}$ as an adjusting one and $c_{1}$ as a fixed one.

\section{Example 4.5.2 (DAB Bank AG AG Inhaber-Aktien o.N.,Figure}

\section{$4.3 .3(\mathrm{a}))$}

In this example we calculate the MBS option price when the strike price is $K=8$, the current stock price $s 0=S_{400}$, the time until expiration is $\tau=\frac{100}{253}$ and the interest rate is $r=0.01$. Under these assumptions we calculate and compare the multinomial Black-Scholes price, classical Black-Scholes price and the option payoff.
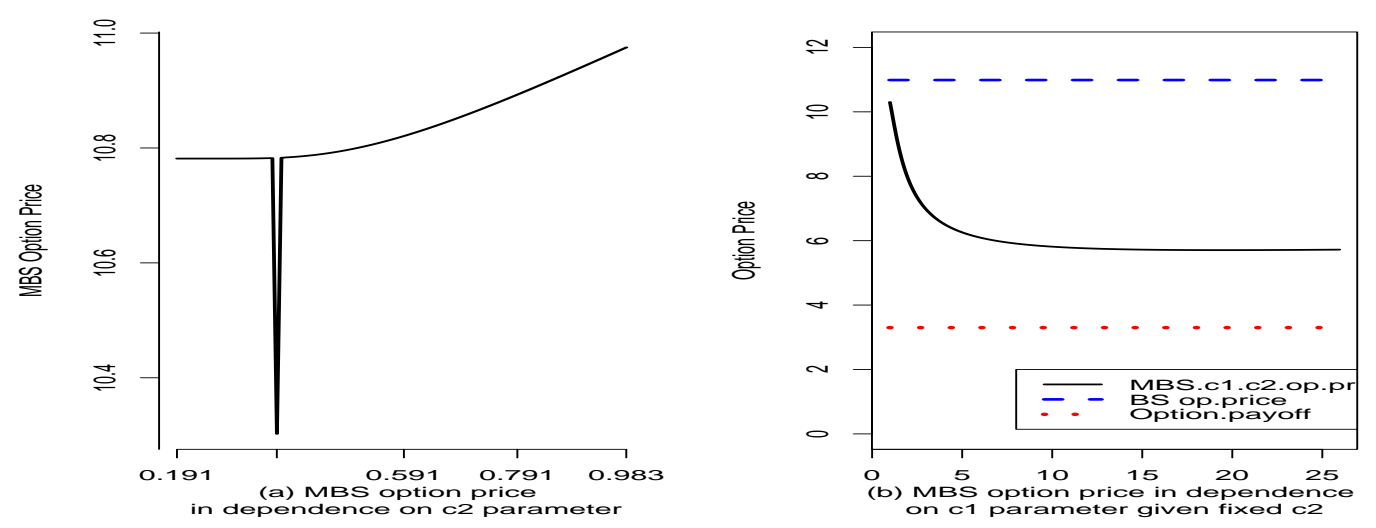

Figure 4.5.2: (a) Multinomial Black-Scholes option price in dependence on the parameter $c_{2}$. (b) Multinomial Black-Scholes option price in dependence on parameter $c_{1}$ given $c_{2}$ (solid line), classical Black-Scholes option price (dashed line), option payoff (dotted line).

The dependence on the parameter $c_{2}$ shown on the Figure 4.5.2 (a) indicates that, in general, the option price increases when $c_{2}$ increases, but there is a specific value of $c_{2}$ when the option price drops down. We hold fixed this value of $c_{2}=c_{2}^{*}$ and next, consider how the option price depends on the parameter $c_{1}$ 
given this $c_{2}^{*}$. On the Figure 4.5.2(b) one can see the multinomial Black-Scholes option price in dependence on the parameter $c_{1}$ (solid line) given the parameter value $c_{2}^{*}$ is closer to the option payoff (dotted line) than the Black-Scholes option price (dashed line).

As one can see from the Figure 4.5.2 (b) the classical Black-Scholes formula and multinomial Black-Scholes model overestimate (which is usual for the markets with high volatility) the option payoff but it is clear, that adjusting the parameters $c_{1}$ and $c_{2}$ the multinomial BS model gives prices which are closer to the payoff than BS prices.

Example 4.5.3 (DCI Datab. Commerce Industry AG InhaberAktien o.N., Figure 4.3.2 (a))

We consider that the strike price is $K=1$, the current stock price is $s 0=S_{400}$, the time until expiration is $\tau=\frac{100}{253}$ and the interest rate is $r=0.05$. Under such assumptions we calculate the multinomial Black-Scholes, classical Black-Scholes and the option payoff.
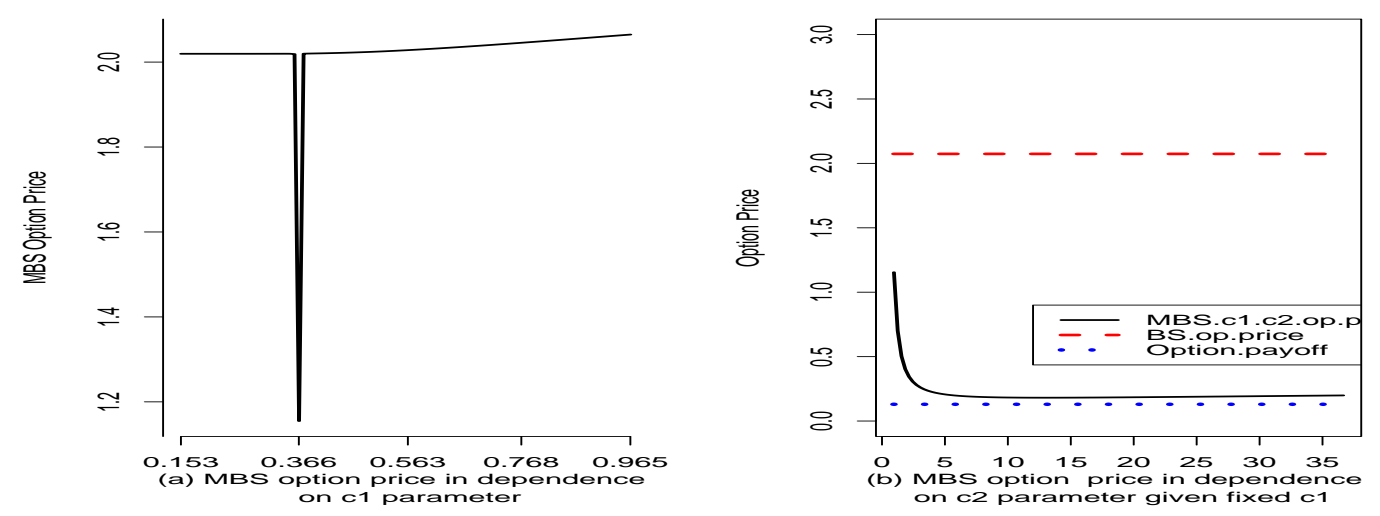

Figure 4.5.3: (a) Multinomial Black-Scholes option price in dependence on the parameter $c_{1}$. (b) Multinomial Black-Scholes option price in dependence on parameter $c_{2}$ given fixed $c_{1}$ (solid line), classical Black-Scholes option price (dashed line) and option payoff (dotted line).

It is shown on the Figure 4.5.3 (a) that there is the value of parameter 
$c_{1}=0.366$ under which the multinomial Black-Scholes option price drops while in other cases it increases as $c_{1}$ increases. Again, we hold fixed this value of $c_{1}$ and see (Figure 4.5.3 (b)) how the option price changes when the parameter $c_{2}$ changes. There are values of parameter $c_{2}$ when the multinomial Black-Scholes option price is closer to the option payoff then the classical Black-Scholes option price. So that properly adjusting parameters $c_{1}$ and $c_{2}$ one can obtain more accurate option prices. 


\section{Bibliography}

[1] Akgiray, V. and Booth, G.G., (1988). The Stable-Law Model of Stock Returns. Journal of Business and Economic Statistics, 6, 51-57

[2] Bachelier, L., (1990). Théorie de la Spéculation. Annales de Ecole Normale Superieure Series 3, 17, 21-86. English Transl. in: Coonter, P.H. (ed.), (1964). The Random Character of Stock Market Prices. Cambridge, MA: MIT Press.

[3] Barndorff-Nielsen, O., (1994). Gaussian-inverse Gaussian processes and the modelling of stock returns, presented at the 2. Workshop on Stochastics and Finance 1994 in Berlin.

[4] Barndorff-Nielsen, O. E., Levendovskii, S., (2001). Feller Processes of Normal Inverse Gaussian type, Quantitative Finance 1, 318-331.

[5] Barndorff-Nielsen, O. E., and Shephard, N., Normal modified stable processes, forthcoming in Theory of Probability and Mathematical Statistics.

[6] Beckers, S. (1981) Standard deviations implied in option prices as predictors of future stock price variability. J. Bank. Finance 5, 363-381.

[7] Black, F., Scholes M. (1973) The pricing of options and corporate liabilities. J. Political Econom. 81, 637-654.

[8] Black, F., Scholes, M. (1972) The valuation of option contracts and a test of market efficiency. J. Finance 27, 399-417.

[9] Bouchaud, J.P., and Sornette, D., J. Phys. I France 4, 863 (1994); Bouchaud, J.P., and Sornette, D., J. Phys. I France 5, 219 (1995). 


\section{References}

[10] Bouchaud, J.P., Iori, G., and Sornette, D., Risk 9(3), 61 (1996).

[11] Bouchaud, J.P., and Potters, M., (1997). Theory of Financial Risk. Paris, Alléa-Saclay, Eurolles.

[12] Boyarchenko, S., Levendovskǐ̀, S., (2000). Option pricing for truncated Lévy processes. International Journal of Theoretical and Applied Finance 3:3, $549-552$.

[13] Boyarchenko, S., Levendovskiì, S., (1999). Generalizations of the Black- Scholes equation for truncated Lévy processes, Working Paper.

[14] Boyarchenko, S., Levendovskiï, S., (1999). Generalizations of the Black- Scholes equation for truncated Lévy processes, Working Paper.

[15] Boyle, P.P., Ananthanarayanan, A. L. (1977) The impact of variance estimation in option valuation models J. Finan. Econom. 5, 375-387.

[16] Chesney, M., Scott, L. (1989) Pricing European currency options: a comparison of the modified Black-Scholes model and a random variance model. J. Finan. Quant. Anal. 24, 267-284.

[17] Chesney, M., Elliott, R., Madan, D., Yang, H. (1993b) Diffusion coefficient estimation and asset pricing when risk premia and sensitivities are time varying. Math. Finance 3, 85-99.

[18] Chiarella, C., Shenhuai Gao, (2002). Modelling the Value of the S\&P 500 - A System Dynamics Perspective. Working paper 115. University of Technology, Sydney.

[19] Cox, J.C., and Ross, S., (1976). The valuation of options for alternative stochastic processes. Journal of Financial Economics, 3, 145-166.

[20] Cox, J. C., Ross, S. A., Rubinstein, M. (1979) Option pricing: a simplified approach. J. Finan. Econom. 7, 229-263.

[21] Duffie, J. D., and Hurrison, J. M. (1993) "Arbitrage pricing of a Russian option and perpetual lookback options", The Annals of Applied Probability 3, no.3, 641-651. 


\section{References}

[22] Eberlein, E. and Keller, U., (1995). Hyperbolic distributions in finance. Bernoulli 1, 281-299.

[23] Engle, R. F., Bollerslev, T. (1986) Modelling the persistence of conditional variances. Econometric Rev. 5, 1-50.

[24] Fama, E., (1965). The Behavior of Stock Market Prices. Journal of Business, $38,34-105$.

[25] Föllmer, H., and Schied, A. (2002). Stochastic Finance. An Introduction in Discrete Time. De Gruyter studies in mathematics 27. Walter de Gruyter GmbH \& Co. KG, Berlin, Germany.

[26] Galai, D. (1977) Tests of market efficiency of the Chicago Board Options Exchange. J. Business 50, 167-197.

[27] Goodman V., Stumpfli, J. (2000) An Introduction to Mathemtics of Finance: Modeling and Hedging. Textbook. Brooks/Cole.

[28] Hull, J. C. (1997) Options, Futures, and Other Derivatives. 3rd ed. PrenticeHall, Englewood Cliffs (New Jersey)

[29] Jaschke, S. R., (2000). A Note on Stochastic Volatility, GARCH models, and Hyperbolic Distributions. Working paper. WIAS, Berlin.

[30] Kon, S.J., (1984). Models of stock returns - a comparison. Journal of Finance 39 , issue $1,147-165$.

[31] Koponen, I., (1995). Analytic approach to the problem of convergence of truncated Lévy flights towards the Gaussian stochastic process. Physical Review E 52, no.1, 1197-1199.

[32] Kramkov, D.O. and Shiryaev, A.N. (1994) "On the rational pricing of the 'Russian Option' for the symmetrical binomial model of a (B,S)-market", Theory of Probability and Applications 39, no.1, 153-162.

[33] Latané, H., Rendleman, R.J. (1976) Standard deviations of stock price ratios implied in option prices. J. Finance 31, 369-381. 


\section{References}

[34] Lau, A.H.-L., Lau, H.-S., and Wingender, J.R., (1990) The Distribution of Stock Returns: New Evidence Against the Stable Model. Journal of Business and Economic Statistics, 8, 217-223.

[35] Lo, A.W. and MacKinlay, A.C.,(1988) Stock Market Prices do not Follow Random Walks: Evidence from a Simple Specification Test, Review of Financial Studies, 1, 41-66.

[36] Madan, D., Milne, F., Shefrin, H. (1989) The mutinomial option pricing model and its Brownian and Poisson limits. Rev. Finan. Stud. 2, 251-265.

[37] Madan, D.B., Carr, P.P., and Chang, E.C., (1998) The variance gamma process and option pricing. European Finance Review 2, 79-105.

[38] Madan, D.B., and Seneta, E., (1990) The variance gamma (V.G.) model for share market returns. Journal of Business 63, no. 4, 511-524.

[39] Malkiel, G. Burton, (1990) A Random Walk Down Wall Street. Norton \& Company. New York.

[40] Mandelbrot, B.B., (1962) Sur Certain Prix Spéculatifs: Faits Empiriques et Modéle Basé sur les Processes Stables Additifs de Paul Lévy. Comptes Rendus, 254, 3968-3970.

[41] Mandelbrot, B.B., (1963a). New Methods in Statistical Economics. Journal of Political Economy, 71, 421-440.

[42] Marsh, T.A., Rosenfeld, E.R. (1986) Non-trading, market marking, and estimates of stock price volatility. J. Finan. Econom. 15, 359-372.

[43] Mellino, A., Turnbull, S.M. (1990) Pricing foreign currency options with stochastic volatility. J. Econometrics 45, 239-265.

[44] Merton, R. C. (1973) Theory of rational option pricing. Bell J. Econom. Manag. Sci. 4, 141-183.

[45] Merton, R. C., (1976) Option pricing when underlying stock returns are discontinuous, Journal of Financial Economics 3, 125-144. 


\section{References}

[46] Mittnik, S. and Rachev, S.T., (1993b) Modeling Asset Returns with Alternative Stable Distributions. Econometric Reviews, 12(3), 261-330.

[47] Motoczyński, M. and Stettner, Ł. (1998) On option pricing in the multidimensional Cox-Ross-Rubinstein model. Applicationes Mathematicae 25, 1, $55-72$.

[48] Musiela, M., Rutkowski, M. (1998) Martingale Methods in Financial Modelling. Springer-Verlag

[49] Nelson, D.B. (1991) Conditional heteroskedasticity in asset returns: a new approach. Econometrica 59, 347-370.

[50] Officer, R.R., (1972). The Distribution of Stock Returns. Journal of the American Statistical Association, 76, 807-812.

[51] Parkinson, M. (1980) The extreme value method for estimating the variance of the rate of return. J. Business 53, 61-66.

[52] Pliska, S. R., (1997). Introduction to mathematical finance: discrete time models. Blackcwell Publishers Ltd, Oxford, UK.

[53] Rachev, S. T., Rüschendorf, L. (1994) Models for option prices, Theory of probability and its Applications 39, no.1, 121-152.

[54] Rejman, A., Weron, A. (1996) Option pricing for hyperboilyc CRR model, Athens Conference on Applied Probability and Time Series, Volume I: Applied Probabiliy, Lecture Notes in Statistics 114, 321-381.

[55] Rendleman, R. J., Bartter,B. J. (1978) Two-state option pricing, Unpublished paper (Graduate School of Management, Northwestern University, Evanston, IL)

[56] Richter, H. (1956) Wahrscheinlichkeitstheorie. Springer-Verlag. BerlinGoettingen-Heidelberg.

[57] Rubinstein, M. (1991) Exotic Options, Working paper no. 220, Institute of Business and Economic Research, University of California, Berkeley, December, 1991 


\section{References}

[58] Samuelson, P.A. (1964) Rational Theory of Warrant Pricing. In The Random Character of Stock Market Prices, Ed. P. Cootner, pp 506 532, Cambridge, MIT Press. 15

[59] Shiryaev, A. N. (1989). Probability.

[60] Shiryaev, A. N. (1999) Essentials of stochastic finance: Facts, Models, Theory. World Scientific Publishing Co. Pte. Ltd.

[61] Scott, L.O. (1987) Option pricing when the variance changes randomly: theory, estimation, and an application. J. Finan. Quant. Anal. 22, 419-438.

[62] Scott, L.O. (1991) Random-variance option pricing: empirical tests of the model and delta-sigma hedging. Adv. in Futures Options Res. 5, 113-135.

[63] Sharpe, W. F. (1978) Investments, Prentice-Hall, Englewood Cliffs, NJ

[64] Shepp, L. A., and Shiryaev, A. N. (1993) "The Russian Option: Reduced regret", Annals of Applied Probability 3, no.3, 631-640.

[65] Shepp, L. A. and Shiryaev, A. N. (1994) "A new look at pricing of the Russian option", Theory of Probability and its Applications 39, no.1, 103-119.

[66] Shiryaev, A. N., Kabanov, Yu. M., Kramkov O. D., and Melnikov, A. V. (1994) "Toward the theory of pricing of options of both European and American types. I. Discrete time", Theory of Probability and its Applications 39, no. $1,14-60$

[67] Shiryaev, A. N., Kabanov, Yu. M., Kramkov O. D., and Melnikov, A. V. (1994) "Toward the theory of pricing of options of both European and American types. II. Continuous time", Theory of Probability and its Applications 39, no.1, 61-102.

[68] Schwert, G.W., Seguin, P.J. (1990) Heteroskedasticity in stock returns. J. Finance 45, 1129-1155.

[69] Skinner, D.J. (1989) Options markets and stock return volatility. J. Finan. Econom. 23, 61-78. 


\section{References}

[70] Teichmöller, J., (1971). A Note on the Distribution of the Stock Price Changes. Journal of the American Statistical Association, 66, 282-284.

[71] Tessitore, G. and Zabczyk, J. (1999) Pricing Options in multiasset and Multinomial model. Preprint 601, Institute of Mathematics, Polish Academy of Sciences.

[72] Wiggins, J.B. (1987) Option values under stochastic volatility: theory and empirical estimates. J. Finan. Econom. 19, 351-372. 


\section{Curriculum Vitae}

19.12.1978

1984-1993

1993-1995

$1995-2000$

15.06 .2000

2000-2003

since 01.10 .2002 born in Tashkent, Uzbekistan

Middle School N252 of Sergeliyski district, Tashkent, Uzbekistan

Middle School N110, class with a mathematical bias, Tashkent, Uzbekistan

Department of Mathematics and Mechanics of St.Petersburg State University, St.Petersburg, Russia

Diploma in Mathematics from St.Petersburg State University

Postgraduate Study at the Mathematics and Mechanics

Department of St.Petersburg State University,

St.Petersburg, Russia

Ph.D. student of Prof. Dr. Manfred Denker,

Institute for Mathematical Stochastics of the University of Göttingen,

member of Interdisciplinary PhD Program "Applied Statistics and Empirical Methods" of the University of Göttingen 